University of Redlands

\title{
Coastal Biophysical Inventory Database for the Point Reyes National Seashore
}

A Major Individual Project submitted in partial satisfaction of the requirements

for the degree of Master of Science in Geographic Information Systems

\author{
by \\ Joseph Guy Kinyon \\ Karen Kemp, Ph.D., Committee Chair \\ Mark Kumler, Ph.D. \\ Victoria Kouyoumjian, M.B.A.
}

March 2010 
Coastal Biophysical Inventory Database for the Point Reyes National Seashore

Copyright (C) 2010

by

Joseph Guy Kinyon 
The report of Joseph Guy Kinyon is approved.
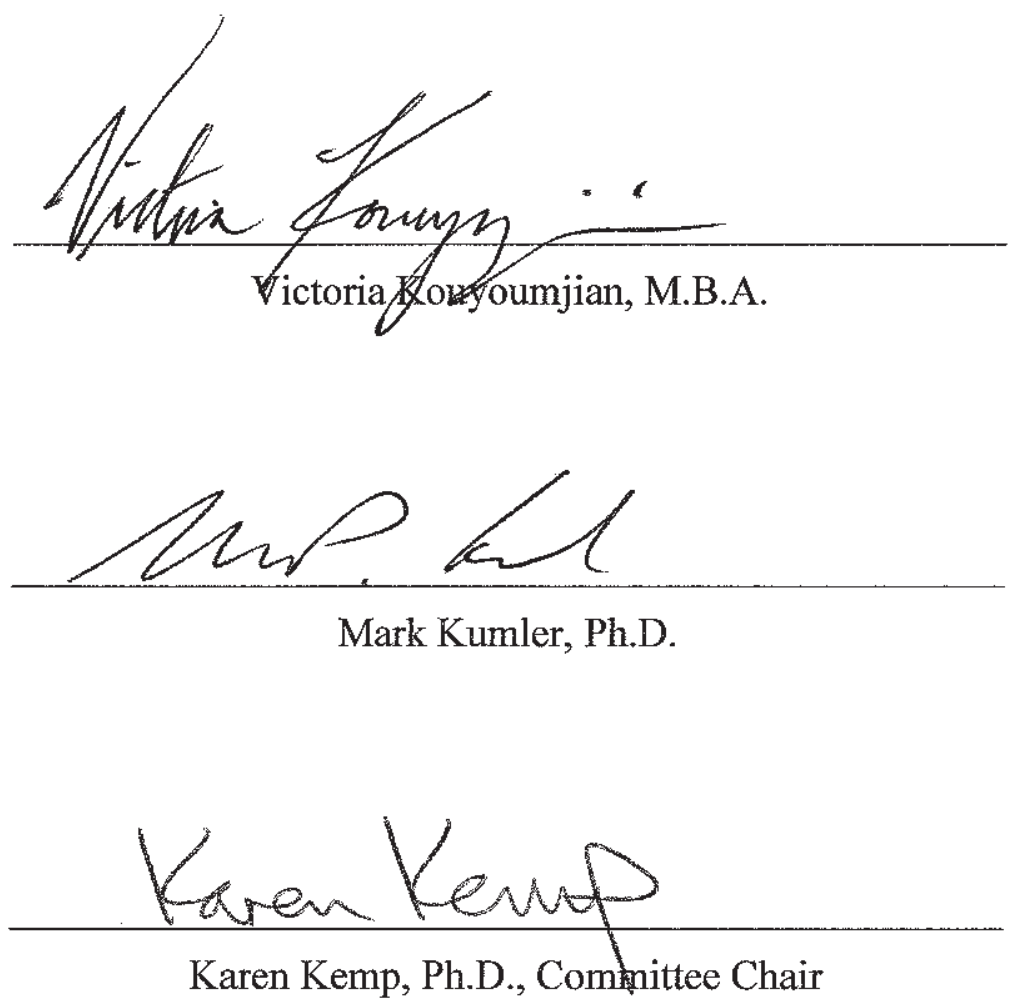

March 2010 



\section{Acknowledgements}

I would like to acknowledge several key people. Without their influence and assistance the completion of the work would not have been possible.

I would first like to thank the National Park Service. In the ongoing challenge to engage people in the enjoyment and protection of our national resources and heritage they see the use of GIS as an essential tool for successful management. Specifically I'd like to acknowledge David Schirokauer as an employee of the National Park Service who is a pragmatic champion of GIS for the completion of this work and the original patron for this project. I'd also like to thank Dr. Benjamin Becker, Director of the Pacific Coast Science and Learning Center who absorbed the continuation of this project into his many responsibilities and helped me see it through.

The completion of this project would not have been possible without the collaboration of PISCO at UCSC. There are many staff members who have received my appreciation for their effort, but Rani Gaddam will never receive enough thanks for her hours of revision, field work and intertidal research expertise.

I would like to thank my committee advisors Karen Kemp, Victoria Kouyoumjian, and Mark Kumler for their direction and mentorship. It is with great difficulty that I write academic papers and their corrections and suggestions for revision recognized this and provided feedback that made it possible to clear this hurdle. There are also philosophical dilemmas in a learning experience with the breadth of this project and their professional advice was of great support to me.

I have had the luxury of the experience and tutelage of many of my professors at the University of Redlands. Some of them I relied upon for assistance far beyond their normal duties. Of these teachers, three must be acknowledged above others. Marinus de Bakker for his conceptualization of project management so that an understanding of it leads to holistic practice of GIS, Thad Tilton who has creatively advised me on too many GIS programming details to begin to enumerate, and Aileen Buckley for the disciplined practice of an aesthetic vision. They rounded out my education to know the details of the parts in order to appreciate the whole and to understand the breadth of the whole in order to recognize the importance of every single part.

Many family and friends rushed to my support when I announced I was pursuing a Master of Science in Geographic Information Systems. They include my parents, who as professionals in their fields have left me with the expectation to aspire to my best, my friends Mat and Anne Rowley who have acted like family in their enthusiastic support, Theresa Ellis for greasing the program's wheels, my colleagues in Cohort 4 who were friends and family while I was there, and finally my dear wife Jennifer Stock who was a pillar of support and patience during this extended project and occasionally outshines the sun with her smile. Because of them, I am eternally grateful that in the circus of life, I have always performed with a net.

Because it would be a great injustice to merely thank the people mentioned above within the boundaries of this page, it is my aspiration to repay the debt of gratitude I have acknowledged here by sharing the support, knowledge and experience I have gained with others in the same enthusiastic way it was mentored to me. 


\begin{abstract}
Coastal Biophysical Inventory Database for the Point Reyes National Seashore

by

Joseph Guy Kinyon
\end{abstract}

The Coastal Biophysical Inventory Database is the repository of the data gathered from a rapid assessment of approximately $161 \mathrm{~km}$ of the intertidal habitat managed by the Point Reyes National Seashore and Golden Gate National Recreation Area. The Coastal Biophysical Inventory Database is modeled after the "Alaska Coastal Resources Inventory and Mapping Database” and CoastWalker program of Glacier Bay National Park and Preserve. The protocol and database were adapted for this effort to represent the features of the Point Reyes National Seashore and Golden Gate National Recreation Area located along the northern central coast of California

The database is an integration of spatial data and observation data entered and browsed through an interface designed to complement the methods of the observation protocol. The Coastal Biophysical Inventory (CBI) and Mapping Protocol is the methodology to collect and store repeatable observations of the intertidal zone to create a baseline of information useful for resource management and potentially assist damage assessment in the event of an oil spill. The inventory contributes to the knowledge needed for the conservation of coastal resources managed in the public's trust.

The Coastal Biophysical Inventory Database is a Microsoft Access 2003 format relational database with a customized data entry interface programmed in Microsoft Access Visual Basic for Applications. The interface facilitates the entry, storage and relation of substrate, biology, photographs, and other field observations. Data can be browsed or queried using query tools common to the Microsoft Access software or using custom spatial query tools built into the interface with ESRI MapObjects LT 2.0 ActiveX COM objects.

The Coastal Biophysical Inventory's GIS data set is useful for collecting, analyzing and reporting field observations about the intertidal zone. The GIS data set is linked to the observation data set through a unique number, the Segment ID, by using the relate tools found in ArcGIS (9.2-10). The Segment ID is a non-repeating number that references a section of coastline that is delineated by the type and form of the substrate observed. The Segment ID allows connection to the biological observations and other observation records such as photos or the original data sheets. Through ArcGIS connections to the observation database using the Segment ID, summaries of biodiversity or habitat can be made by location.

The Coastal Biophysical Inventory has completed its initial goals to assess the coastline of two National Parks. The data set collected provides a snapshot of information and the database allows for future observations to be recorded. It provides coastal resource managers a broad insight and orientation to the intertidal resources managed by the National Park Service. 


\section{Table of Contents}

Chapter 1 - Introduction

$\begin{array}{lll}\text { C.1 Client } & 2\end{array}$

1.2 Problem Statement 2

$\begin{array}{lll}1.3 & \text { Solution } & 2\end{array}$

1.3.1 Goals and Objectives 3

1.3.2 Scope of this document 3

1.3.3 Methods 4

1.4 Audience 4

1.5 Overview 5

$\begin{array}{ll}\text { Chapter } 2 \text { - Background } & 7\end{array}$

$\begin{array}{lll}2.1 & \text { Legacy of the Exxon Valdez } & 7\end{array}$

2.2 Alaska Coastal Resources Inventory and Mapping Database, the Glacier Bay National Park and Preserve's database for the CoastWalker intertidal inventory 13

2.3 Point Reyes National Seashore \& Golden Gate National Recreation Area

implements intertidal inventory $\quad 14$

2.4 Pacific Coast Science \& Learning Center manages project 19

Chapter 3 - Systems Analysis and Design $\quad 21$

$\begin{array}{lll}3.1 & \text { Problem Statement } & 21\end{array}$

$\begin{array}{ll}3.2 & \text { Requirements Analysis } \\ & 21\end{array}$

$\begin{array}{lll}3.2 .1 & \text { User Needs } & 21\end{array}$

$\begin{array}{lll}3.2 .2 & \text { System constraints } & 26\end{array}$

3.2.3 Functional and Non Functional Requirements Summary 28

$\begin{array}{lll}3.3 & \text { System Design } & 28\end{array}$ 
$\begin{array}{llr}3.3 .1 & \text { People } & 29\end{array}$

$\begin{array}{lll}\text { 3.3.2 Software } & 31\end{array}$

3.3.3 Hardware 33

3.3.4 Methods $\quad 35$

$\begin{array}{lll}\text { 3.3.5 Data types } & 37\end{array}$

$\begin{array}{llr}3.4 & \text { Project Plan } & 39\end{array}$

3.4.1 Delineate Scope of work 39

3.4.2 Change order tools for project management 40

$\begin{array}{lll}\text { 3.4.3 Software specifications planning } & 40\end{array}$

3.4.4 Cartographic specifications $\quad 41$

3.4.5 Software programming training plan 41

3.4.6 Pilot Project $\quad 42$

3.4.7 Access Database Development 42

$\begin{array}{lll}\text { 3.4.8 Testing Schedule } & 42\end{array}$

3.4.9 Project Execution Plan 42

3.4.10 Project Documentation 43

$\begin{array}{lll}3.5 & \text { Summary } & 43\end{array}$

Chapter 4 - Database Design 45

4.1 Access Database $\quad 45$

4.1.1 The role of the segment $\quad 47$

4.1.2 The Observation Records 48

4.1.3 Data Integrity 51

4.1.4 Domains $\quad 54$

4.1.5 Non tabular data $\quad 55$

4.2 GIS Database $\quad 55$ 
4.2.1 Conceptual Data Model $\quad 55$

4.2.2 Logical Data Model 56

Chapter 5 - Database Interface Design 63

5.1 Interface design inside a parent application $\quad 63$

5.2 Guidelines to CBI database interface design 63

5.3 Representing the multiple observations of a single record $\quad 64$

$\begin{array}{lll}5.4 & \text { Interface design and user feedback }\end{array}$

$5.5 \quad$ Organization of Database interface $\quad 65$

$\begin{array}{lll}5.6 & \text { Summary } & 65\end{array}$

$\begin{array}{lr}\text { Chapter } 6 \text { - Data } & 67\end{array}$

$\begin{array}{llr}6.1 & \text { Data sources } & 67\end{array}$

$\begin{array}{lll}\text { 6.1.1 Collection and Acquisition } & 67\end{array}$

$\begin{array}{llr}6.2 & \text { Data Scrubbing and Loading }\end{array}$

6.2.1 Metadata management issues 69

6.2.2 Preparing spatial data fundamental to the project 70

$\begin{array}{lll}\text { 6.2.3 } & \text { Reference Imagery } & 71\end{array}$

6.2.4 Data conversion for a flexible workflow $\quad 72$

$\begin{array}{lll}\text { 6.2.5 Subsets of larger data } & 73\end{array}$

$\begin{array}{ll}\text { Chapter } 7 \text { - Implementation } & 75\end{array}$

$\begin{array}{lll}7.1 & \text { Training } & 75\end{array}$

$\begin{array}{lll}7.2 & \text { Pilot Project } & 76\end{array}$

7.3 Coastal Biophysical Inventory, Visual Basic 6 version 80

7.4 Coastal Biophysical Inventory, Visual Basic for Application version 88

7.4.1 Feature requests by users, an example of software programming 88 
7.4.2 Programming around error, an example of one solution 90

7.4.3 VBA and VB 6, the combined solution to interface and installation 91

$\begin{array}{lll}\text { 7.4.4 Data entry } & 92\end{array}$

Chapter 8 - Results and Analysis $\quad 96$

Chapter 9 - Conclusions and Future Work 101

$\begin{array}{lll}\text { 9.1 New and unexpected uses for the CBI } & 101\end{array}$

$\begin{array}{lll}9.2 & \text { Anticipated evolution of the dataset } & 102\end{array}$

$\begin{array}{ll}\text { Works Cited } & 105\end{array}$

$\begin{array}{ll}\text { Appendix } & 111\end{array}$ 


\section{Table of Figures}

Figure 1. Comparison of the Exxon Valdez Oil Spill extent to California coastline. 12

Figure 2. National Park Service Lands of the CBI ................................................. 15

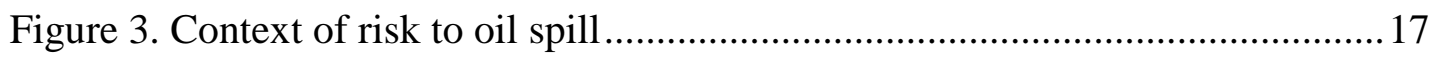

Figure 4. General Use Case Diagram .............................................................. 24

Figure 5. Nested container database analogy ................................................... 47

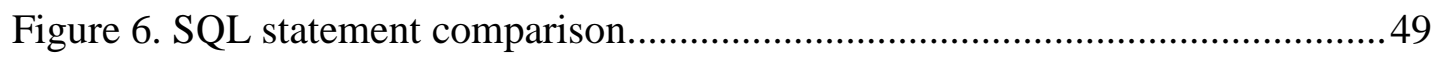

Figure 7. Database relationships diagram snapshot...............................................53

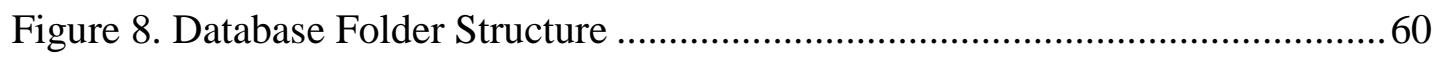

Figure 9. California Hummingbirds prototype interface screenshot, map tools........77

Figure 10. California Hummingbirds prototype interface screenshot, data display .. 78

Figure 11. California Hummingbirds prototype interface screenshot, reference....... 79

Figure 12. DataRepeater custom ActiveX object substrate display ..........................81

Figure 13. Repeating forms display for substrate in Access VBA ........................... 82

Figure 14.CBI VB6 interface, map page screenshot............................................. 82

Figure 15. CBI VB6 interface, segment observation screenshot ............................ 83

Figure 16. CBI VB6 interface, biology page screenshot ..................................... 84

Figure 17. CBI VB6 interface, substrate page screenshot ....................................85

Figure 18. CBI VB6 interface, protocol and documentation page screenshot...........86

Figure 19. CBI VB6 interface, lookup table and image viewer page screenshot ......87

Figure 20. CBI database interface, Access VBA version screen shot 1 ....................94

Figure 21. CBI database interface, Access VBA version screen shot 2 ...................95

Figure 22. Comparison of system versions and interface design emphasis.............100 


\section{List of Tables}

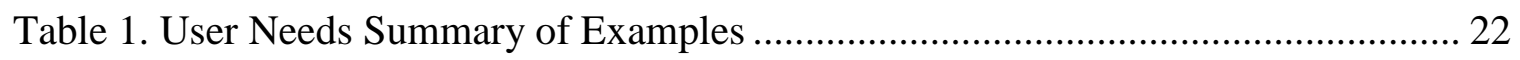

Table 2. Data types, their anticipated uses and management for system design .............. 38

Table 3. Data functional parameters and reasoning for database design........................ 46

Table 4. Feature class properties for CBI Segment .................................................. 56

Table 5. CBI Segment feature class attributes and data types .................................... 57 



\section{List of Acronyms and Definitions}

\section{Barycenter:}

CCRP:

CBI:

COM:

Double hull:
The center of mass. For the Moon and the Earth, it is the center of mass of both bodies which they lopsidedly revolve around approximately every 28 days. Because the Earth is so much more massive than the moon, the barycenter of this pair is below the Earth's surface. The center of the earth and Moon revolve around this point, which is different than their axis of rotation. Centripetal acceleration causes the Earth and moon to bulge on the surfaces (water surfaces too) furthest from the barycenter as they whip around this point in space as it travels around the Sun. The California Coastal Records Project is the labor of love of Kenneth and Gabrielle Adelman who fly the length of the California coast in a helicopter and document it through photographs. Using a GPS coupled to the camera, coordinates of the location the photo was taken are embedded in the header of the digital image file. They repeat the flight approximately every 2 years so that changes to the coast can be made by comparing photos. Through the internet, the Coastal Biophysical Inventory Database links to the set of oblique aerial photographs which were taken closest to the observation records being review via a link to the CCRP website on the data entry interface. Coastal Biophysical Inventory, the project name for the National Park Service's effort to characterize the substrate, organisms and general description of intertidal habitat into a database of observations with coordinates and photographs for each observation location. The observation protocol used employs rapid assessment of coastal conditions using pragmatic taxonomic precision and repeatable classification of substrate. Now that the project is complete, many users refer to the resulting GIS and Relational Database by the term, rather than meaning the whole project and protocol.

Component Object Model is an interface standard of Microsoft used for communication and programming object creation. COM in this project refers to Microsoft ActiveX programming objects and they were used primarily in the Coastal Biophysical Inventory for the creation of desktop applications such as the data entry interface for the database.

Also called dual hull. A technique of ship construction where a boat is essentially built inside a boat, creating an exterior hull that navigates and is exposed to damage and an interior hull that contains and protects the cargo. The reasoning is that in an accident such as a collision, environmental catastrophe can be avoided if a poisonous cargo is not released because the outer hull takes the brunt of the damage and leaves the inner hull functionally 
FGDC:

intact. The Oil Pollution Act of 1990 mandated that single hull tankers, single hull with double sides and single hull with double bottom be phased out of operation in the United States Exclusive Economic Zone (EEZ) between 1995 and 2010. Double hulls do not protect from explosion, ship breaking or major collisions because those incidents penetrate both inner and outer hulls. Flexible inner hulls have been proposed as a remedy. Double hull is confused with dual-hull a term also used to describe multi-hull construction where the hulls are side by side and parallel as found in outriggers, catamaran ships and high speed passenger ferries, Federal Geographic Data Committee. An interagency committee to promote the coordination of geospatial data within the United States Federal Government and is under the Office of Management and Budget. It establishes geospatial data management standards including metadata standards that organizations such as the NPS are obliged to use and is designed align with the International Organization of Standards metadata standards.

Garbage Collection: A software and operating system process that recovers and reorganizes system resources such as system memory (RAM) so that when variables used by a software application are no longer needed they can be prioritized for use by the application or the memory is released for use by other applications. If software includes many variables that are part of looped or iterative logic in the code and release and reuse of the memory does not occur, then memory available to the application quickly increases in size as it makes requests for new blocks of memory. If variables no longer in use are not recycled or released from use, eventually the allocation of memory resources to the software can starve the operating system of resources and that causes dramatic performance problems such as system failure.

GGNRA:

GIS:

GOGA:

Golden Gate:
Golden Gate National Recreation Area

Geographic Information System, the people, computer hardware, software, data and methods that collect, organize, store, analyze and report information related to locations on the Earth.

Golden Gate National Recreation Area, the National Park

Service's 4 letter park code, usually made by the first four letters of the park's name or the first two letters of the first two words in the park's name if it has a multi word name, e.g. GOlden GAte. Often used by NPS staff instead of GGNRA.

The area at the entrance to San Francisco Bay including the southern end of the Marin Peninsula and the northern end San Francisco Peninsula. It includes the Golden Gate Strait, an approximately one mile wide and three and a half mile long strait between the bay and the Pacific Ocean. It was named Chrysopylae (Golden Gate) by Captain John C. Fremont (circa 1846) of the U.S. Army Topographical Engineers as an allusion to the Chrysoceras 
GPS:

IEEE:

Intertidal Zone:

NOAA:
(Golden Horn) of Istanbul, Turkey. The famous Golden Gate Bridge derives its name from the passage it spans.

Global Positioning System. A tool for approximating location in a coordinate system by measuring the time a radio signal travels from multiple known locations (e.g. satellites, reference stations, and high accuracy networks) compared to a highly accurate reference clocks at each of those locations. A variety of equipment and methods for synchronizing clock calculations improves accuracy and precision of measuring the elapsed time of radio signal travel and therefore improves the accuracy and precision of measuring the location of the receiving antenna. Correction of error introduced into the signal along its path is a major component of a GPS. A constellation of multiple satellites allows worldwide coverage of radio signal. Methods and technology allow measurements of location with error less than a centimeter. The GPS used in the Coastal Biophysical Inventory had a standard of accuracy of less than 1 meter $95 \%$ of the time.

Institute of Electrical and Electronics Engineers is an international organization that advances electrical technology and promotes the dissemination of consensus-based industry standards for voluntary use or adoption to improve communication, quality, safety, function and interoperability through technology.

The area of a coastline defined by the high and low elevation of the water level during the tidal cycle. The intertidal zone varies in height based on latitude, alignment of the Sun and Moon, and the shape of the ocean basin (other physical factors play minor, but measurable parts in changing the tide). The intertidal zone is divided and classified by the biological zonation that occurs relative to the elevation of the tides at a given location. Substrate type is a key component to the intertidal zone's form, e.g. silt grades at a shallow slope which can create an intertidal zone miles wide with 2 meters of elevation change in the ocean surface, while rocky intertidal or piers can approach vertical (and surpass vertical to create overhangs) making the width of the intertidal zone almost equal to the maximum tidal elevation change. Silt creates a stable volume for intertidal habitat and bedrock surface creates a relatively permanent substrate for individual or colonial sessile animals that can affix to if for decades. Sand, pebble and cobbles allow some interstitial and motile organisms but rarely support sessile or encrusting organisms due to the scouring of their surfaces during wave action and tumbling in the surf. For this reason, the term "intertidal zone" is often preceded by a term identifying the main substrate type discussed, e.g. rocky intertidal. National Oceanic and Atmospheric Administration. A United States federal agency focused on the conditions of the oceans and atmosphere. Part of the Department of Commerce, NOAA includes 
NPS:

\section{Objects:}

PAH:

PATA:

PCSLC:

PDF: many smaller agencies such as the National Marine Sanctuaries, National Geodetic Survey, National Marine Fisheries and the National Weather Service.

National Park Service. A United States federal agency that is a bureau within the Department of the Interior tasked with caring for special places protected by the citizens of the United States so that all can experience its heritage. The Pacific Coast Science and Learning Center, Golden Gate National Recreation Area, Point, Reyes National Seashore, San Francisco Area Inventory and Monitoring Program are all participants in the Coastal Biophysical Inventory that are part of the National Park Service. While parks of national or federal status existed for decades prior to 1900, the National Park Service was initiated with the National Park Service Organic Act of 1916.

Modular and interacting software code connected together to create software through programmed routines and events. Objects are usually instantiated from an abstract class.

Polycyclic aromatic hydrocarbons are chemical compounds found naturally in unrefined oil that have been shown to be carcinogenic, mutagenic and teratogenic.

Parallel Advanced Technology Attachment. A subsystem (computer bus) between computer components inside a computer or between computers that is local in that it has physical and prioritized connection directly to the CPU and therefore improved connection of peripherals to the computer hardware system. A technology specialized for use in the connection of adapters to mass storage devices such as hard disk drives.

The Pacific Coast Science and Learning Center is one of approximately 20 centers that promote research and education in National Parks. The PCSLC works to promote science based research in the San Francisco Bay Area Network of Parks (SFAN) which includes Golden Gate National Recreation Area (GOGA), Point Reyes National Seashore (PORE), Muir Woods National Monument (MUWO), Fort Point National Historic Site (FOPO),, Presidio of San Francisco (PRES), John Muir National Historic Site (JOMU), Eugene O’Neil National Historic Site (EUON), and Rosie the Riveter / World War II Home Front National Historic Park (RORI). The PCSLC works closely with the SFAN Inventory and Monitoring Network.

Portable Document Format is an electronic document standard established by Adobe Systems Incorporated derived from the PostScript language. In the CBI, Adobe Acrobat Reader is used to view the contents of the PDF files created to store the images of the original "raw" field data sheets, supporting documents, protocol, and guides for the Coastal Biophysical Inventory. 
Pilot Area:

PISCO:

PORE:

PRNS:

PRNSA:

SCSI:

SATA:

SQL:

SWOT:
A location at the mouth of a bay or strait where expert navigators assume control of large ships and pilot them into ports. The pilots meet the vessels at a predetermined location (located on nautical charts) and board the incomming vessel.

Partnership for Interdisciplinary Studies of Coastal Oceans. The University of California at Santa Cruz (UCSC) is a member of PISCO. PISCO at UCSC has a group of staff skilled in the field identification of intertidal organisms and the logistics behind such field work called the Intertidal SWAT Team. The National Park Service collaborated with the PISCO-UCSC team of researchers for collecting Coastal Biophysical Inventory data between 2006 and 2008.

Point Reyes National Seashore, the National Park Service's 4 letter park code, usually made by the first four letters of the park's name or the first two letters of the first two words in the park's name if it has a multi word name, e.g. POint REyes. Used by NPS staff instead of PRNS.

Product Life Cycle: A description of the software development process that outlines the planned stages a software will go through from origin to obsolescence. In a waterfall model of the cycle, the software usually progresses through the following planned stages:

- Requirements

- Design

- Implementation

- Verification

- Maintenance

- Deprecation

Point Reyes National Seashore

Point Reyes National Seashore Association, a nonprofit partnered with the Point Reyes National Seashore to provide support to meet missions and goals of the park's general management plan.

Small Computer System Interface

Serial Advanced Technology Attachment, A subsystem (computer bus) between computer components inside a computer or between computers that is local in that it has physical and prioritized connection directly to the CPU and therefore improved connection of peripherals to the computer hardware system. The standard is specialized for the connection of adapters to mass storage devices such as hard disk drives.

Structured Query Language is a standardized language of commands to interact with relational databases and tables. An initialism and mnemonic to organize the four components to risk analysis to identify risk for management. The acronym comes from the risk analysis tasks of assessing Strengths, Weaknesses, Opportunities, and Threats to the success of a project. SWOT 
assessment is scalable to every level of a project management plan's risk analysis. 


\section{Chapter 1 - Introduction}

Heritage is essential to national identity, however brief. Personal identity often overlaps with one's national identity, one might argue it is integral. National identity is tied to its geography (Hooson 1994). When people identify events or places that define their heritage, they are saying more than making a simple recognition of a special event or feature. They are often showing greater aspirations of who they hope to be or the opportunities they wish to preserve for their children (Duncan and Burns 2009).Without the ancient places and cultural antiquities that created the heritage that world leaders of Europe respected, the developing United States connected its heritage to natural wonders. If a place was marked by tragedy or hardship, then its preservation served as a physical lesson of the challenges that people can overcome and as a moral if they are mindful of their weaknesses or vulnerability. National Parks that preserve natural wonders and historic places became a significant part of the United States' cultural heritage.

The heritage of the United States is one of expansion of emigrating cultures across a continent from prehistoric times. There are places that are of such serenity or magnificence they invoke joy and humble awe and there are others that release overwhelming memories. There are events memorialized by places that make one question the hearts of men and women or give one hope that the people may be as grand as the landscape they call home. Because these places and events are part of one's national identity, they become part of one's personal identity. Some places are singled out because they conserve or preserve the wild beauty of the United State's origins, the lessons learned at great cost, the opportunities for renewal, or the homes of special individuals who have inspired and created a significant part of the nation. National Parks are the special places singled out as representative places of a nation's heritage, and by extension, its people (National Park Service n.d.). If such a place is damaged, then a nation's heritage is damaged. For this reason, the expectations for conservation and preservation of National Parks are high-these are places that affect many people deeply.

How does one recover a damaged heritage? The Coastal Biophysical Inventory attempts to answer part of that question by addressing the question "how do we prevent or begin to recover from damage to a place that has been set aside with national importance?” For National Parks that include bays or oceans, the enormous environmental catastrophe that can be caused by oil spills is a daily risk as ships pass by. The ships that contain millions of gallons of chemicals that are either toxic to life or poisonous to the character of the place that sustains people. This is a special combination of great risk and great consequences.

The Coastal Biophysical Inventory is a project that promotes an initial step towards organizing and sharing information about the intertidal zone in Point Reyes National Seashore and Golden Gate Nation Recreation Area to create a reference for comparison. The project described in this paper aspires to make large amounts of information for a large area organized in such a way that its ease of access promotes decision making that is either timely (responding quickly to disaster) or with foresight (responding with anticipated information). If it can prevent damage from an oil spill, then its value is difficult to measure. If it can help respond to an oil spill by assessing damage and facilitating restoration of part of a National Park, then it fulfills its basic goals. Its 
simplest goal is that it allows a coastal resource manager at a national park to derive knowledge and patterns about an area they steward by summarizing a rich set of details.

\subsection{Client}

The client for this project changed over the duration of the project. David Schirokauer, the GIS Manager and Biologist for the Point Reyes National Seashore, was the initial client. David Schirokauer moved to Klondike Gold Rush National History Park in Alaska during the project, and passed the project to the Pacific Coast Science and Learning Center. The California Department of Fish and Game Oil Spill Prevention and Response team provided additional project funding to complete the project to the Pacific Coast Science and Learning Center. Joseph Kinyon, GIS \& Biodiversity Database Manager at the Pacific Coast Science and Learning Center became project manager for the Coastal Biophysical Inventory and assumed the role of client for the remainder of the project. The client in the broadest terms is the Point Reyes National Seashore and Golden Gate National Recreation Area coastal resource managers with reporting obligation of the results to the California Department of Fish and Game Oil Spill Prevention and Response team who provide additional funding support.

\subsection{Problem Statement}

The coastline of the Point Reyes National Seashore and Golden Gate National Recreation Area is at risk to catastrophic damage. Evaluating that damage and staging the success for its repair is difficult to impossible without a baseline of knowledge. An inventory of the biological and geological components creates a baseline of data that also encourages additional research. It is necessary in resource management, planning and emergency response that the data be quickly accessed and efficiently viewed by record and selectable by location for timely and effective decision making that affects a place.

The Coastal Biophysical Inventory requires a storage and retrieval system for field observations and spatial information related to the area of observation. The user interface and database structure stores the field observation protocol and enhances the entry of data from field data sheets into a Microsoft Access database

Limited budget and availability of specific software licenses require developing a solution that is freely distributable without additional royalty payment or licenses required by the end user or the purchase of additional developer software or programming tools.

\subsection{Solution}

The solution proposed was to adapt the existing Coastal Biophysical Inventory Database (housed in Microsoft Access 97 database file) to include a data entry form that allows spatial querying and selection using ESRI MapObjects LT 2.0 for the mapping interface. An existing interface using Microsoft Access Visual Basic for Applications and database from the Glacier Bay National Park and Preserve's CoastWalker provided a working example. For budget and licensing constraints, the resulting database and interface use software available on National Park Service computer workstations as a standard installation running Windows XP Professional and Microsoft Office XP (2002) 
Professional. The map portion of the interface display uses MapObjects LT 2.0 ActiveX controls and the image database portion of the interface uses Ammara DBPix ActiveX controls. To use the database interface a onetime installation of the supporting files is required to operate the software. The interface required that spatial data and tabular data be organize so that it can be accessed programmatically through the custom interface software of the Access database data entry form.

\subsubsection{Goals and Objectives}

The objective of the Coastal Biophysical Database Project is to adapt the database currently in use by the Point Reyes National Seashore that was inspired by the Glacier Bay National Park and Preserve. Included in this objective is the goal to enter data, view data, and query data with spatial tools created from software and custom programming objects that can be distributed without additional licensing costs in a format that can "stand alone" in its operation and organization of data.

David Schirokauer was the biologist and GIS manager at Point Reyes National Seashore who was the client who initiated the project. His project goal was to adapt the existing database to use a map display for data selection and browsing as the Glacier Bay National Park and Preserve had done,

"Specifically, I'd like to implement Map Objects within an access database to display geographic information in an MS Access database form. We have all the associated GIS data but I suspect it will take a fair amount of work to implement this in Microsoft Access. Glacier Bay National Park in Alaska has completed a similar project and we are modeling our coastal inventory after theirs” (Schirokauer 2003).

The Coastal Biophysical Inventory Database project for Point Reyes National Seashore attempts to embrace the functions of the Coastal Resources Inventory and Mapping Database of Glacier Bay National Park and Preserve in order to provide the repository for ongoing inventory of the intertidal zone over the next five to ten years and allow quick data entry, data browsing and viewing for decision support by resource managers during and after the inventory project.

With a project of this size and variety of data, documentation of the methods of data collection and data presentation is essential for ease of access by users unfamiliar with the methods or data. Documentation of the project is essential to promote the repeatability of the observations in the same manner which is necessary for comparisons made to observe change. The documentation is managed as metadata and will need to be accessible with the data as reference. If an observation is repeatable using the same protocols, then the observations of change will not require interpolation of meaning or methods that could be overly complex for quick response.

\subsubsection{Scope of this document}

This document reflects only a portion of the scope of the Coastal Biophysical Inventory project and the work completed. This document reflects the work accomplished to complete the project goal of creating an integrated database and interface. This document covers the development and design of the observation and spatial information storage and graphical user interface and the unanticipated problems encountered that required additional solutions. Protocols, manuals, and project documentation that show the 
additional work or projects that were essential to complete the Coastal Biophysical Inventory Database are attached as appendices. Those projects were beyond the original goals of the Major Independent Project and are excluded from the scope of this document, but are presented as references because their content does provide important context to design decisions.

\subsubsection{Methods}

Collaboration was the most essential method to complete the project. Creating tools or processes that support or promote collaboration between different elements of the project and the staff that completed those elements was essential to the successful management of the project. The design of the database reflected input from the users so that it could deliver the interface that facilitated the completion of work within the Coastal Biophysical Inventory system.

Programming design and testing explored different languages (e.g. Visual Basic 6, Visual Basic for Applications, and VB.Net), and programming environments to create a software product that met the basic software licensing limitations outlined at the beginning of the project and responded to additional resources becoming available later in the project. Because programming skills at the beginning of the project were limited, additional education was required to learn the programming required skills and pilot projects were programmed to demonstrate functional capabilities and the constraints to programming solutions.

Database design employed conceptual user models, database modeling software, modification of existing databases and creation of a new database based upon the lessons learned from pilot projects and the iterative feedback gained from programming the data entry interface. During the database creation, changes to the project and unsatisfactory performance required strengthened alignment with the field data protocol. Final database design enhanced proper functioning that responded to changes in newer versions of the Microsoft Office Access software and Windows operating systems.

The development of the spatial data into a GIS required two parallel tracks of design. One method organized the data into a hierarchical folder structure that was fixed in organization to support programming methods and collection of data while programming continued. The other method organized the spatial data so that it could be accessed by GIS software external to the project. The result was a hybrid of the methods that supported both functions. The methods of design were to assess user needs, explore solutions, implement test solutions, iteratively revise, create final solution and tune the results using user feedback or error analysis.

\subsection{Audience}

This project was designed to serve the needs of GIS and coastal resource managers of the National Park Service. The project anticipates use by professionals in different agencies, however the primary goal of this project was to facilitate data entry by field biologists trained in the taxonomy of marine organisms of the eastern Pacific Ocean, in recording field observation and for data review by biologists or GIS managers in federal or state agencies that are familiar with oil spill damage in the intertidal zone. The majority of users of the project will have an undergraduate education in biology or greater via the 
observations database interface. Users of the GIS data set will have training in GIS methods and software. Readers of this document should be familiar with basic relational database design and object oriented programming in order to comprehend the depth and breadth of the design challenges and the attempted solution that were used to create the underlying structure of database files and user interface forms. A familiarity with ESRI GIS software products, especially ArcGIS, and with GPS technology will assist in the comprehension of essential design choices that were made to create the GIS dataset.

\subsection{Overview}

This document begins with an introduction to the Coastal Biophysical Inventory for the Point Reyes National Seashore. The introduction, Chapter 1, describes the client and change of client over the course of the project. It includes the scope of this document to describe the methods and objectives to reach the goal of creating a database of observations of the intertidal zone for Point Reyes National Seashore and Golden Gate National Recreation Area.

The introduction is followed in Chapter 2 by description of the origin of the National Park Service's goal to rapidly assess the conditions of the intertidal zone. This chapter includes the context to risk of oil spill that the coastline faces and why it is urgent to collect a baseline of information. It follows the historical lineage of the project and management of the project up to the project being managed by the Pacific Coast Science and Learning Center.

In order to adequately provide a solution to the needs of the National Park Service, an analysis of conditions and use was made that identified the different users of a database and software that would store and retrieve the rich set of data created by the researchers following the observation and mapping protocol. Throughout Chapter 3 the specifications for the database and interface are outlined as are the functional requirements for the system to work. The operating environment, the system specifications and the functional requirements drove the system design which addresses all the major components that create a GIS. The systems analysis and design concludes with a plan to manage the project to create the system and database that solved the NPS's need for a database and interface for the Coastal Biophysical Inventory.

Chapters 4 and 5 cover the parts of the Coastal Biophysical Inventory that are part of the scope and emphasis of this document which is the design of the tabular database, the GIS database and the graphical user interface. There are many parts of the Coastal Biophysical Inventory that were completed to support the three major design elements, however they will not be discussed except in reference or context to the design goals and are provide as electronic references summarized in the Appendix.

There was a tremendous amount of data collection and data managed for this project. Chapter 6 describes the work done to gather the observation data stored in the database and the transformation and manipulation of existing spatial data to populate the GIS database with reference layers.

Implementation of the project is discussed in Chapter 7. The project included iterations of the database and interface including a pilot demonstration project, the first version of the planned database and interface as a standalone software, and a the final database and interface created in Microsoft Access based upon the emphasis of the design and needs of the project as they changed over the project's duration. 
The final parts of this document explain the project after its completion. These parts include the ongoing maintenance of the database, data entry, anticipated uses, possible improvements, and its retirement. The document ends with lessons learned and hindsight that might benefit others with a similar endeavor. 


\section{Chapter 2 - Background}

The Coastal Biophysical Inventory was intended to be a rapid assessment in order to gather repeatable observations for a large area with as much detail as possible within the logistical constraints of available low tides, adequate satellite coverage for GPS measurements, day light hours and access to shoreline. There are ongoing long term monitoring sites at multiple rocky intertidal areas in the Point Reyes National Seashore and the Golden Gate National Recreation Area, however an assessment of all the intertidal zone shoreline with consistent effort was needed to add a broad understanding of the intertidal resources to the deep understanding gained from monitoring. The need for a rapid assessment was born from the urgency created by the perceived risk to oil spill. This risk has been highlighted by multiple oil spills around the world, along the northern central coast of California and in or adjacent to San Francisco Bay. The risk drives a need for a rapid assessment protocol. The rapid assessment protocol structures the type of data collected. The type of data collected drives details of database design. Data retrieval, data entry and summarization of observation data in the database guide the interface design for the software which was the focus of this project. The following history creates the context for the Coastal Biophysical Inventory up to the time when the author of this project joined.

\subsection{Legacy of the Exxon Valdez}

The Exxon Valdez Oil Spill on March 26, 1989 was one of the worst environmental tragedies wrought by human negligence, but the spill size of approximately 10.8 million gallons of Prudhoe Bay crude oil ranked as the fifty-third largest oil spill in the world only a decade later (NOAA/ Hazardous Materials Response and Assessment Division 1992). It is hard to measure the "largeness" of a spill and can be estimated as volume of oil spilled, extent of spread or resulting damage. Volume of a single spill event is used in this document to define largest. By comparison, the Gulf War oil spill in 1991 is estimated to be the largest human source of oil released at 446,600,000 gallons. The remote Alaska coast line with complex currents, northern storms, and inadequate emergency response infrastructure made response and clean up difficult (Alaska Oil Spill Commission, the State of Alaska 1990).

Oil containment booms that were part the emergency response plan intended to be in place on the ocean's surface, corralling any spill, inside of five hours did not arrive for twelve hours. The oil spilling from the ship was not contained until fifty nine hours had passed, and the majority of the oil was already spilled and continued to spread. In less than twenty days the crude oil spread a linear distance of well over four hundred miles (Alaska Department of Fish and Game 1993). Storm winds of up to seventy miles per hour two days after the spill and seasonally extreme tides up to eighteen feet dispersed the oil high up the beaches and down into the (usually submerged) subtidal zone along approximately thirteen hundred miles of shoreline (See 1 for a map of oil dispersal from the Exxon Valdez spill).

An icon in the pantheon of environmental catastrophes, the Exxon Valdez Oil Spill was especially devastating to the intertidal zone. The intertidal zone is an 
ecosystem defined by the flux in the margin between the terrestrial and the marine environment. When the terrestrial and marine ecosystems intermingle in the intertidal zone, the biodiversity is stunning, and when combined with the nutrient rich upwelling found along the Eastern Pacific Ocean, the intertidal zone becomes a breathtaking example of fecundity. Because water is denser than oil (with a few exceptions), oil floats upon the surface. In the Exxon Valdez Spill, currents and crashing waves repeatedly delivered the surface borne oil to the intertidal zone. Organisms were smothered, exposed to toxic levels of crude oil (and additional chemicals contained in the oil e.g. PAH) (Hostettler, et al. 1999), reproductively impaired, isolated from food, and physically disabled. Twenty years later, significant deposits of Exxon Valdez oil persist immediately below the surface substrate in the intertidal zone (Exxon Valdez Oil Spill Trustee Council 2009).

The intertidal zone is a dynamic environment affected by processes of a planetary scale. The large basin shape and volume of the Pacific Ocean allows for a significant change (at the human scale) in the tidal height at the continent's edge due to the sun and moon's gravitational attraction to the ocean competing with the Earth's gravity for the ocean's mass. On the opposite side of the earth is a bulge that is created by the centripetal acceleration of the ocean's mass caused by the spinning of the earth and moon around its barycenter (extended towards the sun) and a lagging in displacement of the ocean's mass due to less gravitational attraction by the moon at the increased distance. Along the Pacific Ocean's margin this combination of forces means that a location on the coast is spun into approximately two bulges and two depressions in the ocean's surface every twenty four hours as the Earth rotates on its axis, resulting in a high or low tide every six hours. For these reasons, a large surface born oil spill can be re-deposited on a beach twice daily, highlighting the need for rapid response. The range of the rise and fall of the tides varies in height as the distance from the equator increases and as the sun and moon change positions throughout a month. Along the California coast the maximum vertical change in sea level due to tides is approximately 8 feet, creating an 8 foot tall ribbon of intertidal coastal habitat from Mexico to Oregon that is vulnerable to the type of damage seen along the Alaskan coast from the Exxon Valdez spill.

The geology along the California coast is the heterogeneous remnants of the subduction of ancient tectonic plates, ancient archipelagos accreted along the continental crust, eroded plutons, eruptions, transport of rock material and the deposition of rock material by currents or crustal movement over eons (McPhee 1994). This diverse geology interacts with the wave action in the intertidal zone --weathering and eroding differently for each geological type and therefore creating extensive geomorphological diversity in the intertidal zone. The resulting steep bedrock cliffs and headlands, sandy broad beaches, pebbles, cobbles, silt and the occasional human structures built on top are a few examples of the substrate that can be encountered at low tide. Each type of substrate responds to an oil spill differently due to its shape and size. Because of the substrate type, some shorelines shed the oil and can return to near pre-spill conditions quickly while others can sequester oil deposits interstitially with damaging effects on the populations of both sessile organisms and those that seasonally use the intertidal zone as a breeding or nursery zone.

Oil is currently an integral part of our economy. Centralized refineries require the transport of the unrefined oil to the processing facilities and then transport again from the 
refinery as the refracted petroleum product and byproduct are carried to the markets that consume them. More petroleum product consumption means the continued transport of crude oil from wells via pipeline, truck and ship. More consumption implies more transport (Transportation Economics and Management Systems, Inc 2008). More transport statistically points to greater spill risk. The National Parks along the Pacific Coast are often near shipping lanes (transportation law dictates that oil tanker lanes are no less than fifty miles off the coast) (U.S. Department of Transportation Maritime Administration 2008) and are situated at the mouths of bays and straits where urban population (and voting) centers exist.

The urban populations are built up around historic shipping and commerce centers (for example the San Francisco Bay Area) that were protected harbors and these populations have also supported the creation of local parks nearby. The deep harbors that facilitate international shipping using large vessels are also the location of centralized oil refineries in order to refine the product near the port so that the companies can ship the derived petroleum product elsewhere without additional transport during refinement. Because of the beauty and unique physical features at the transition of bays to the ocean and the strategic location for forts and military reservations that have gained historic significance and limited settlement, National Parks are often near inlets of straits and bays (See figure 3 for a overview of the location of the Coastal Biophysical Inventory, the participating parks and the adjacency of likely risk or sources for potential oil spill).

The entrances to bays are where the protected bays transition from calmer conditions to the forces of the ocean interacting with rugged, remote and occasionally treacherous coastline. Shipping traffic to bulk petroleum transfer ports is limited to the shipping lanes permitted through narrow passages, and sometimes requires extensive training and expertise to navigate. In some cases, the ship's captain temporarily gives their controls to local expert captains called bar or harbor pilots as a way to reduce risk of accident due to the special challenges to navigation to some ports. Due to oil transportation being nearby, coastal National Parks are at greater risk to the damage of the resources they are mandated to protect because the risk of shipwreck can be greater near them.

While U.S. National Parks budgets are funded at a national level by tax dollars, the individual parks are integrally tied to their location and the local economy. Visitor volunteer support and visitor fees are an important way that some parks balance the real cost of management with Congressional budget appropriations. Therefore, when oil spills cause a cascading economic disaster as the Exxon Valdez spill did in the communities (McCammon 2003) surrounding Prince William Sound that were not tied to the petroleum industry (e.g. fishing, tourism/park visitors, and real estate) (McDowell Group 1990), the damage additionally hampers the economic resiliency of a National Park to a similar disaster. It creates an economic crisis that can sometimes eliminate access to essential services that support recovery for the park and are important to supporting park staff whose budgets and projects are taken over by disaster response and whose lives and families that are woven into the fabric of that local economy placed in peril.

During the recent economic recession, petroleum consumption has slowed compared to the previous ten years; however, if efforts to improve the economy are successful then we should expect more energy consumption in the near future (U.S. Energy Information Administration 2009). Oil will continue to be a major source of 
energy consumed even though renewable, non-petroleum based energy technologies race to compete with oil as the energy source. Because replacement energy sources to oil are not universally in place, an increase in energy consumption by a rebounding world economy will likely result in the need for more oil transport which is tied to the available tanker fleet.

Improved tanker ship design with redundant engines, maneuvering thrusters on the bow, redundant control systems, more accurate computer navigation support, and double hull designs suggests that some of these modern ship systems can fail by half and still maneuver (Welch 2003). With one layer of steel separating cargo from the ocean, single-hull tankers like the Exxon Valdez are more vulnerable to spilling oil than double hull tankers if they strike a reef, iceberg, or each other and were required by the U.S. Congress to be phased out by 2015 (Committee on Commerce, Science and Transportation, United States Senate, 108th Congress, First Session 2003).

Only ships that have double hulls to help reduce the risk of spill due to a collision may operate in U.S. waters after 2015. It is important to note here that tankers that transport between U.S. ports, e.g., between Valdez in Alaska, Puget Sound in Washington, and San Francisco, Los Angeles and San Diego in California, are regulated by the Jones Act and must be built in U.S. ship yards (U.S. Department of Transportation Maritime Administration 2008). A slowing economy has slowed the orders for all ship construction from 2007-2009, meaning that companies have been delaying their construction. Shipyards have foreseen a bottleneck in production due to limited construction space for ships of that size if orders are made to supply enough double hull ships by the deadline to accommodate current tonnage shipped (United States General Accounting Office 2000).

Because it is anticipated that there will be a shortage of shipping capacity soon after the deadline, a shortage of tankers means an unwelcome risk to energy security for the U.S. For these reasons, interim waivers to cabotage laws and the Jones Act that restricts oil shipment between U.S. ports to U.S. built ships have already been made that allow for the use of foreign-built, single-hulled ships until the supply of U.S. constructed double hull ships can match the demand in an emergency. As economic stakeholders, U.S. maritime industry has demanded that these waivers have more specific language to ensure they are not used in an overly broad way when emergencies are loosely described (Allegretti, et al. 2005).

Other countries that have experienced far larger spills are already enacting and enforcing double hull regulations keeping aging and outdated ships out of their waters much earlier than the deadline of 2015 in the United States (Cox and Alemanno 2003). For this reason, it is expected that the risk that single hull oil tankers currently pose elsewhere will migrate to U.S. shipping lanes while enforcement of double hull regulations are delayed (Transportation Economics and Management Systems, Inc 2008). In 2015, those who have waited twenty five years for the reduction of risk envisioned by double hull tanker laws soon after the Exxon Valdez oil spill may continue to wait. When combining the anticipated increased need for oil with the delayed double hull regulation deadlines in the United States of America while other countries tighten their hull regulation deadlines (Carlos and Brey 2002) the likelihood that budget minded shipping companies will try to meet revived oil transportation demands with existing single hull ships or emergency waivers suggests that risk of a spill due to a single hull tanker wreck 
in U.S. waters may increase around 2015, contradicting the goals of the Oil Pollution Act of 1990 (United States Congress 1990).

Since the Exxon Valdez spill, coastal resource managers have seen greater legal protections, improved navigation technology, improved shipping protocols, improved methods of damage assessment, and a shift in consciousness about the dependence upon oil. However, the transportation of oil continues in some of the same ships operating over twenty years ago and may be phased out but not replaced (Devanney 2006). Monetary resources to plan for or respond to disaster have been impinged upon by a down turn in the global economy affecting oil spill response services in California (Oil Spill Prevention and Response Technical Advisory Committee, California Department of Fish and Game 2009), and other states, Punitive deterrence or financial restitution from past spills have not been as successful as hoped (Ott 2008). Recent grants inspired by the Cosco Busan spill response have released funds to purchase oil spill equipment in San Francisco Bay Area and were instrumental in containing the Dubai Star Spill in San Francisco in November of 2009 (Singleton 2009). Unfortunately, funding and public support for prevention and response often occurs soon after a disaster happens rather than before.

Coastlines in the National Parks are often remote, rugged, or difficult to access, and are often adjacent to the paths used by shipping lanes and ships transporting petroleum products. Coastal resource managers at the National Parks were pragmatic and began documenting the coastal resources their parks in order to evaluate and quantify patterns of change or damage. The Exxon Valdez spill was much more immediate and provoking to the National Parks in Alaska whose shorelines were either impacted by the spill or were parks that did not have damaged resources but the parks' staff saw the crisis unfold as they lent assistance. One of these parks was Glacier Bay National Park and Preserve whose protocol assisted the efforts at Point Reyes National Seashore and Golden Gate National Recreation Area. 


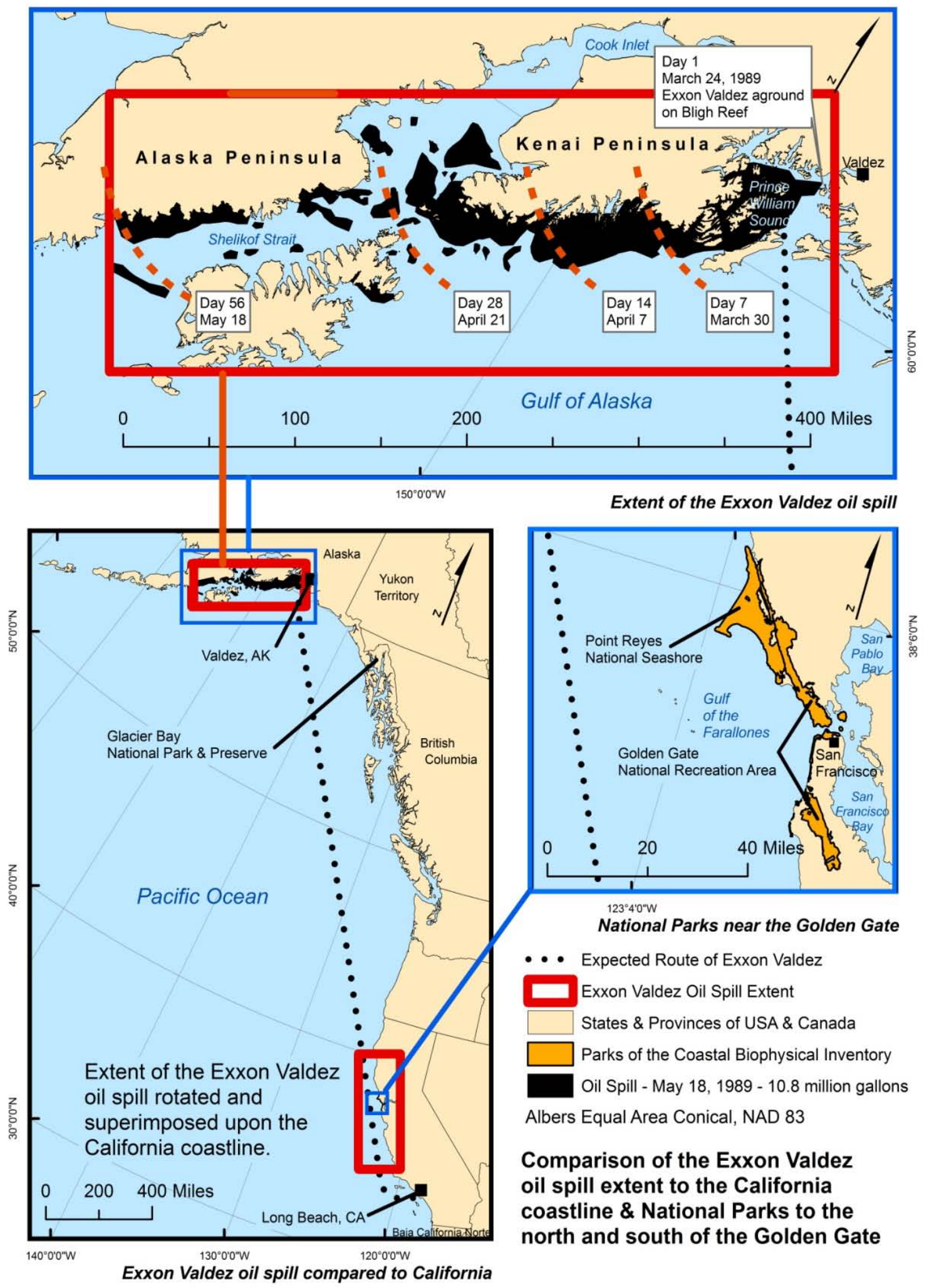

Figure 1. Comparison of the Exxon Valdez Oil Spill extent to California coastline 


\subsection{Alaska Coastal Resources Inventory and Mapping Database, the Glacier Bay National Park and Preserve's database for the CoastWalker intertidal inventory}

Alaskan resource managers were overwhelmed by the devastation of the Exxon Valdez. Research and restoration was hampered by a lack of observations before the spill, leaving them without baselines for their restoration efforts or to gauge oil spill remediation success. Without a baseline for comparison, it is difficult to defend the estimates of damage scientifically or in court when seeking restitution for observed damages. Without this information, seeking emergency funds to respond to a disaster or injury from an oil spill can be difficult. Coastal resource managers took close notice of this dilemma and it inspired several extensive inventories by the National Parks with coastline to mitigate those potential problems. Lewis Sharman of Glacier Bay National Park and Preserve (GLBA) in Alaska acknowledged the precarious place our ignorance places us when managing the risk of our coastal resources,

"As managers, we are humbled by how little we know of the coastal resources under our stewardship in National Parks in Alaska. We have a very poor understanding of what those resources are, not to mention their distributions, abundances, and ranges of variation over time and space. We are charged with preserving and protecting these resources in an unimpaired state, yet it is impossible in most cases to determine whether they have been compromised because it is so very difficult to distinguish between "impaired" and "unimpaired" due to our lack of basic knowledge. Even if a catastrophic event occurs (e.g., Exxon Valdez Oil Spill), and it is clear that coastal resources have been damaged, how can we know when they have "recovered" following restoration activities? In order to fix something that's broken (and decide if and when it's been fixed), we need to know what it looked like and how it worked before it broke!" (Sharman, et al. 2002)

In 1994, coastal parks in Alaska had the opportunity to respond to the National Park Service’s “Coastal Program Needs and Development Survey” and in doing so consistently identified coastal inventories as a priority to establish a baseline inventory and for monitoring change. The National Park Service made funding available for such an undertaking in 1995 to the Alaska Parks region, and GLBA submitted their proposal "Protocol Development for Integrating Coastal Resources Information into Coastal GIS."

A few methods of field data collection were attempted as GLBA. Staff narrowed the methods and revised workflows to collect substrate observations and location measurements. The two workflows that were considered for final use differed fundamentally in their conception of space. One method conceived of the coast as a route represented by a line and collected points to mark the distance along the shore line feature data set they had traveled (dynamic segmentation) while assigning attributes to the reach of the shoreline, and the other was the delineation of beaches into coastal "segments" from aerial orthophotographs as polygons and then adjusting the polygon shape and location of the polygon feature based upon field observations. It is important to note the principal methods behind the creation of a segment, because the Coastal 
Biophysical Inventory protocols adopted by the Point Reyes National Seashore (PORE) and Golden Gate National Recreation Area (GOGA) in California were modeled from the Glacier Bay National Park and Preserve Protocols.

The segmentation of the coastline by distance from an origin was simple for computing and adjusting as a series of related locations, but was difficult to conceptualize and track in the field when the coastline split and when small islands were included. GLBA adopted the method of creating coastal "segments" by delineating the polygons that represent a type of substrate by first digitizing substrate types from aerial photographs and then refining the resulting polygons with field observations. This is similar to other coastal assessments such as the Shore-Zone methodology developed in British Columbia, but did not extend into the subtidal or terrestrial habitats (Howes 2001).

The component of the protocol developed at GLBA that persists in the Coastal Biophysical inventory today is the tenet that the assessment must be rapid and repeatable using a minimum of tools. This is most clear in the substrate classification protocol. The GLBA CoastWalker protocol classifies the coastline by substrate, creating breaks or new segments wherever the substrate changes. The classified substrate is the organizing concept, employing a hierarchy of substrate observations to describe that section of coastline before moving on to the next tasks of taking GPS measurements of where the substrate appears to transition to another and an inventory of organisms observed on the substrate type.

Lewis Sharman and Bill Eichenlaub at GLBA developed a Microsoft Access1997 database to enter and review the observation data with an embedded spatial data browser and image viewer. To avoid paying repeated royalties on a software and database they planned to distribute for free, they chose to use ESRI MapObjects LT 1.0 to create the map component on the database entry form that enabled browsing of the observations by location. ESRI's MapObjects allowed royalty free distribution (ESRI 2003) of their custom software and could be manipulated by the Visual Basic for Applications programming environment built into the Microsoft Office suite of tools.

\subsection{Point Reyes National Seashore \& Golden Gate National Recreation Area implements intertidal inventory}

A problem for resource managers at Point Reyes National Seashore and Golden Gate National Recreation Area is assessing damage to the intertidal zone. The miles of wild and often inaccessible shoreline that is adjacent to the intended Exxon Valdez route between Valdez, Alaska and Long Beach, California (See Figure 1 for a map comparing the expected route of the Exxon Valdez and spill extent to the California coast), means that there is an almost impossible task of assessing the current coastal resources if another oil spill occurs. In addition to this, prioritizing emergency cleanup efforts based on sensitivity of habitat, uniqueness of habitat, and difficulty in restoration are time-critical decisions that are difficult to make with existing data because the data are diverse, not readily accessible, and not interoperable. 


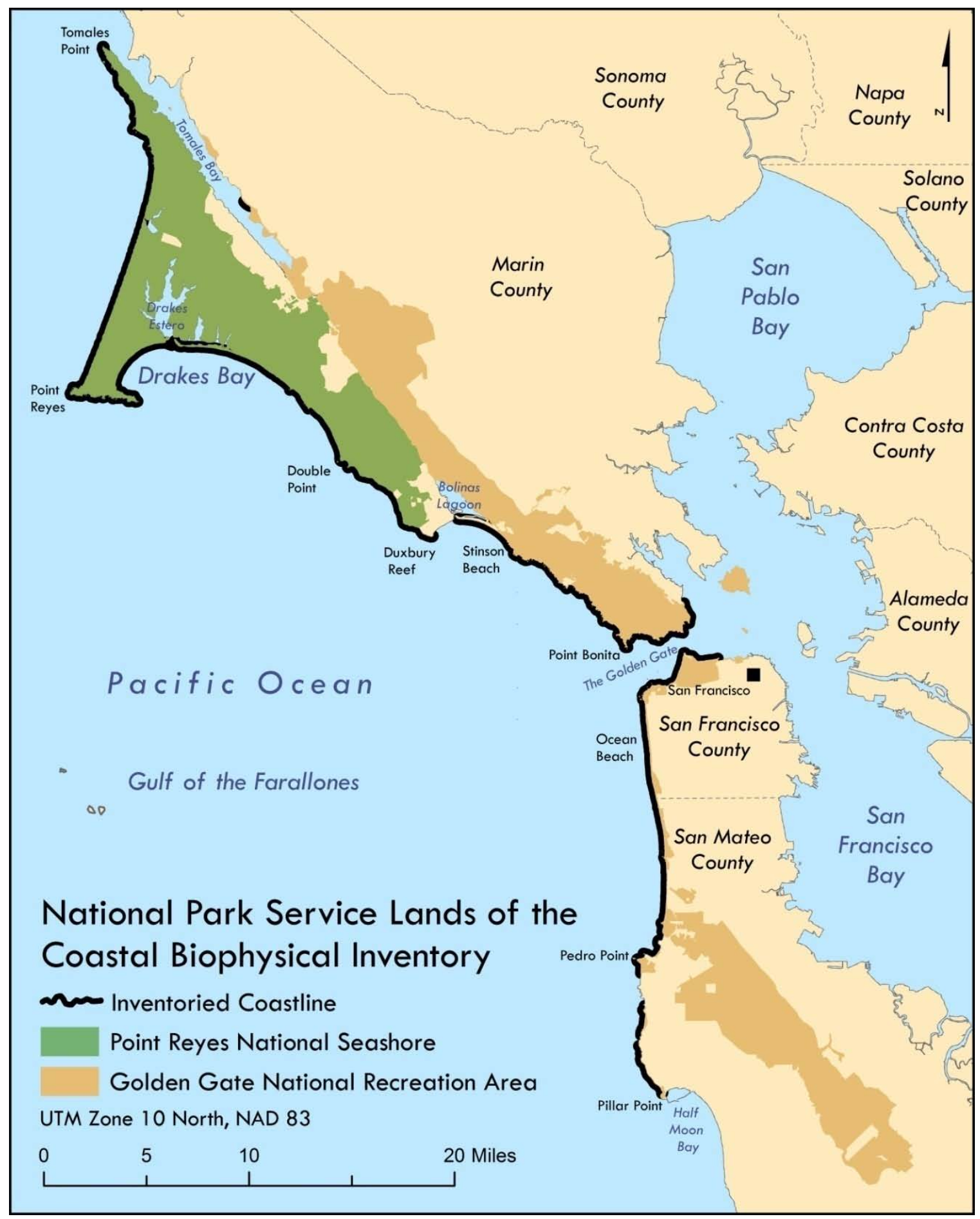

Figure 2. National Park Service Lands of the CBI 
Coastal resource managers at the Point Reyes National Seashore and the Golden Gate National Recreation Area (these parks are part of the National Park System under the United States Department of the Interior, see the extent of these parks in figure 2) recognized that when another chemical spill occurs near the Golden Gate it will have swift impact as pointed out in this National Resource Defense Council (NRDC) oil spills in San Francisco Bay summary of facts:

“A spill the size of 1989's Exxon Valdez disaster in Alaska would reach from Mendocino to San Diego if it occurred off the California coast, and would spell disaster for wildlife here, just as it did in Alaska. In fact, a simulation by the Army Corps of Engineers showed that a spill only about one-tenth the size of the Valdez -- 1.3 million gallons -occurring at the Golden Gate would reach the shores of the Golden Gate National Recreation Area, the Marin shoreline, Angel Island State Park, and the San Francisco waterfront in about six hours” (Natural Resources Defense Council 2001).

Ongoing oil spills from accidents, illegal releases, and aging sunken ships with still oil on board remind resource managers that a huge spill is possible on a regular basis. In addition to oil, other chemicals transported along the coast are of such a toxic nature that clean-up and recovery could prove impossible if disaster is not prevented. Raw sewage released from cruise ships and invasive species larvae in the ballast water of ships are particularly severe threats to the intertidal and bay ecosystems that can be as devastating to an ecosystem as a chemical spill.

San Francisco is famous for the Golden Gate Bridge and the beauty of the Golden Gate, a mile wide gap between the rugged coast of the Marin Headlands and the San Francisco Peninsula that the bridge spans and derives its purpose. It is a historically treacherous entrance to a bay for ships. Darkness of night, thick fog, narrow maneuverability, restricted military zones, enormous tidal currents, bridge piers, and a rocky shoreline of submerged hazards make the daily passing of oil tankers and chemical transport ships from some of the nation's largest refineries an increased risk for disaster. Oil spills in this area span almost a century. In one of the first major oil spills in the area 2,730,000 gallons of bunker fuel spilled from the Frank H. Buck when it struck the steamer the President Coolidge near the entrance to the Golden Gate at night under heavy fog in 1937. In 1971 the oil tankers Arizona Standard and the Oregon Standard collided below the Golden Gate Bridge, spilling approximately 860,000 gallons of oil which spread out to the outer coast more than 30 miles to the north and south within a day, oiling what is now the Point Reyes National Seashore and Golden Gate National Recreation Area. The Puerto Rican exploded and sank 20 miles west of the Golden Gate in 1984, releasing approximately 200,000 gallon. In 1988, 432,000 gallons leaked from Shell Oil's Martinez refinery into the bay when a drain valve was opened. A long history of spills in the area and heavy and regular shipping traffic indicate that the risk of oil spill is present and the consequences large.

Between shipping lanes and the National Parks on the coast (a distance of approximately 50 miles along the coast and less than .5 mile in straits and passages) there 


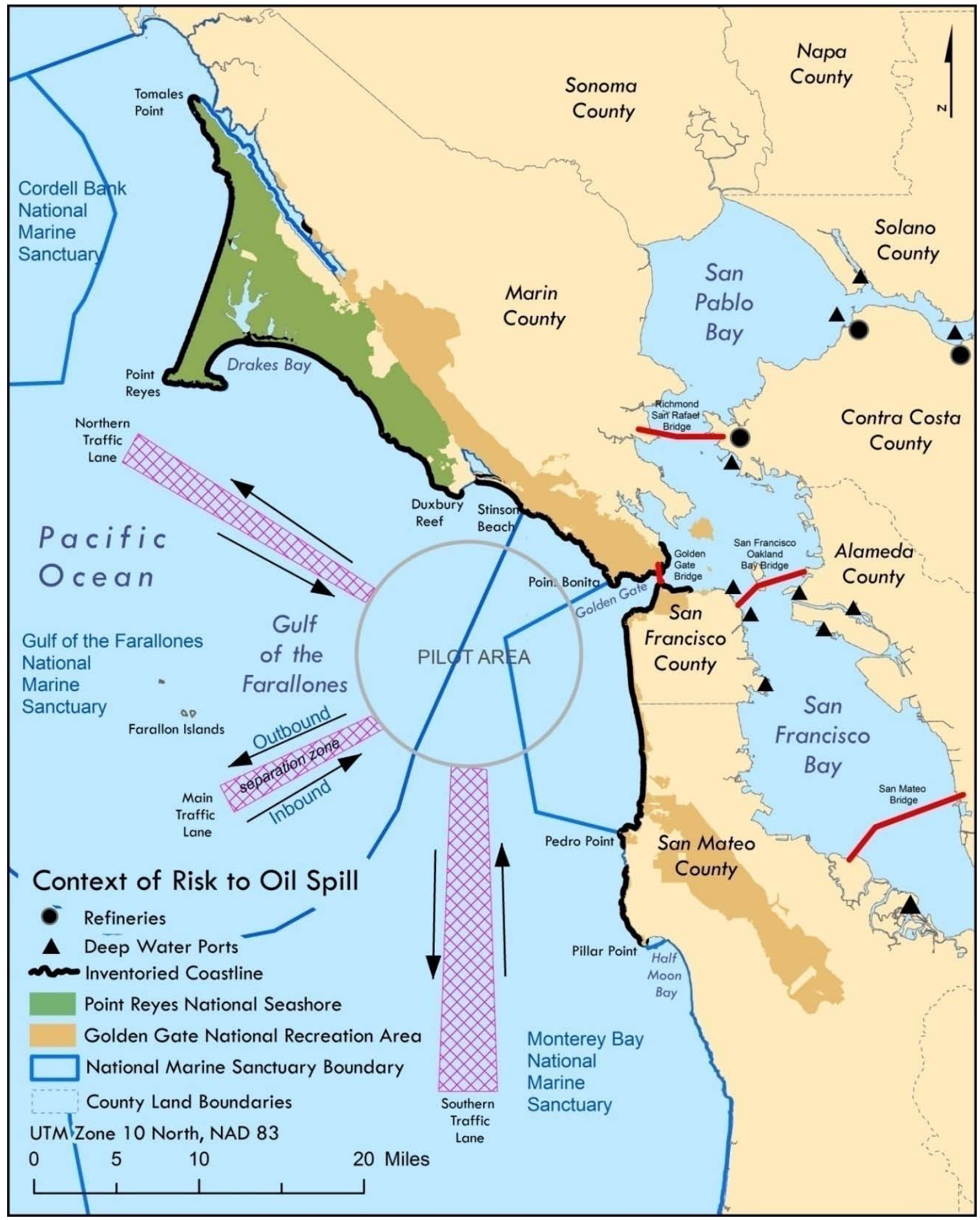

Figure 3. Context of risk to oil spill (shipping lane information from NOAA chart "Point Sur to San Francisco" $31^{\text {st }}$ edition, June 2005) 
are three National Marine Sanctuaries, two of which share shoreline with the National Parks. When organizing for congressional declaration as a sanctuary, a risk assessment of spills in the area was included in its site characterization for Monterey Bay National Marine Sanctuary (MBNMS) which is adjacent to both the Point Reyes National Seashore and the Golden Gate National Recreation Area,

"Available risk assessments indicate that under continued tanker traffic patterns another large oil spill in MBNMS in the next 20 years is not unlikely. The federal Minerals Management Service prepared oil spill risk assessments for the west coast in 1980 and 1988. According to these studies, for ships bound from Alaska to L.A. [Los Angeles] there is a 53\% probability of a spill of 1000 barrels or more, and a $26 \%$ probability of a spill 10,000 barrels or more. According to this risk assessment, by the year 2016 a spill of 1000 barrels or more is more likely to occur than not, and such a spill may occur in the MBNMS (Townsend and Glazer 1992)[Sic]

NOAA's recent assessment of vessel traffic in the MBNMS substantiates concerns about oil spills. NOAA determined that within the MBNMS, the tugs and barges entrusted with assisting tankers in narrow straits are not subject to safety inspections. Existing oil spill response personnel and equipment are insufficient. The craggy, rocky topography of the central coast would make cleanup from a catastrophic oil spill very difficult; even with the best possible response, up to $60 \%$ of the oil would remain in the environment. MBNMS resources such as sea otters, seabirds, and tidepool organisms are particularly sensitive to spilled oil in catastrophic and chronic levels, especially at Pillar Point, Santa Cruz, Elkhorn Slough, Monterey Bay, Carmel Bay, and Point Piedras Blancas.” (Monterey Bay National Marine Sanctuary 1999)

The probabilities quoted in the MBNMS site characterization appear to have been verified multiple times with oil spills of that size since the probabilities were first published in 1994 (Townsend and Glazer 1994). In 1996 the tanker vessel the Cape Mohican spilled 96,000 gallons were spilled while in dry-dock, of which approximately 40,000 gallons entered the San Francisco Bay and exited the bay with tidal currents. In 1998 the tanker vessel Command released approximately 3,000 gallons of intermediate bunker within ten miles of the Golden Gate when departing for Panama. The most recent spill over 1,000 gallons occurred when the cargo vessel Cosco Busan struck the Bay Bridge between San Francisco and Oakland and spilled approximately 58,000 gallons of bunker fuel from its fuel holds. Tidal currents carried oil out the Golden Gate and oil and debris appeared north and south in the intertidal zone in the Golden Gate National Recreation Area and Point Reyes National Seashore, and reached locations approximately twenty five miles to the north and south on the outer coast (exact timeline, dates and locations of the Cosco Busan oiling are currently limited due to ongoing analysis of damage and pending litigation). The risk of damage from a single oil spill and the possibility that it will occur again in the near future still seems likely.

The risk of oil spill to the coastline is clear. The resource managers of the Point Reyes National Seashore and Golden Gate National Recreation Area understood the need for an intertidal inventory for their parks and saw the field work and protocols of Glacier Bay CoastWalker as a program to emulate. In collaboration with researchers at the University of California at Davis, Project designers at Point Reyes National Seashore adapted the protocol to the biology and physical characteristic of the California coast. The ethnographic component of the database and the roll film management process of the 
Glacier Bay CoastWalker protocol did not translate to the ethnological resources or equipment of the California parks, and were not emulated. Different species and the inclusion of elements relevant to resource management agendas at the Point Reyes National Seashore were folded into the protocol. The database was adapted to receive and store the data particular to the California Coast intertidal zone. The data collected for the CBI before this Main Independent Project started was stored in a Microsoft Access 1997 database adapted from the Alaska Coastal Resources Inventory and Mapping Database which was produced by the staff at Southeast Alaska GIS Cluster of National Parks (Sharman, et al. 2002).

\subsection{Pacific Coast Science \& Learning Center manages project}

In 2005, management of the Coastal Biophysical Inventory was transferred from the Point Reyes National Seashore to the Pacific Coast Science and Learning Center (PCSLC) David Schirokauer, the Coastal Biophysical Inventory's project manager and “client” for the Main Independent Project (MIP) represented in this document, took a position at Klondike Gold Rush National Historical Park. This meant that management of the project coincidently became the responsibility of the GIS Manager, Joseph Kinyon, who is also the author of this document. At that time the author became both the client and consultant for this project, which is an unusual circumstance and can present problems with scope that are difficult to resolve. The PCSLC has its office at Point Reyes National Seashore which made the transfer of project administration straightforward due to it being part of the National Park Service with a mission to support science research in the parks represented by the $\mathrm{CBI}$ and because it already shares the same computer network. The next step was to complete the inventory of approximately 80 kilometers of remaining shoreline.

While the administration of the project was an easy transfer, it required recreating partnerships and clarifying protocol essential to its completion. The California Department of Fish and Game's office of Oil Spill Prevention and Response (OSPR) provided a grant to complete the remaining coastline, and therefore had stipulations of reporting. A report due to OSPR upon completion required documentation with detail beyond what had been completed for the project prior. The inventory and mapping protocol in use at the time in was not completed as a report until June of 2005. See the "Protocol" directory of the database for an archived version of this protocol.

During the training to prepare to collect more data it was revealed that Partnership for Interdisciplinary Studies of Coastal Oceans (PISCO) staff could not re-create the previous effort using the existing protocol description. The protocol as written was inadequate as a guide for new field staff uninitiated in the protocol methods, Also, the protocol could not assist with software or database design details to develop solutions for uses beyond the database as it functioned at that time. Through collaboration with PISCO, a revised protocol was created to work as a user manual with sufficient detail to support field work and database design. It also made documentation of the project details essential to facilitate the collaboration. The time to enhance the protocol document delayed new field work for approximately a year and kept revisions and improvements to the database in limbo. In spite of the delay in the project, the project benefited from the revisions and enabled the field work to continue. Through the collaboration with PISCO to improve the protocol document, both PISCO and PCSLC staff learned the protocol 
methods far deeper than if they had been simply read. The thorough understanding of the whole protocol and the details of each step facilitated making pragmatic changes to improve the project workflow and anticipate any cascading effects the changes might make. It also assisted and with solving new problems encountered during the project when some locations were identified that could not be safely accessed. Please see Appendix A for a full version of the protocol document. Additional Data sheets and workflow documents that complement the protocol can be found in the "Protocol" directory of the data set or linked through the "Documentation" page of the database user interface.

The following chapters that detail systems analysis and database design begin with the original project design goals and then note where the transfer of project management described above required the adaptation of the database and of the design to reflect new or different user needs. Many design constraints came from the anticipated use by the agencies described in this background in order to understand the coastal resources they are obliged to protect and either prevent or respond to oil spill damage. Many of the design solutions required the adaptation of the original system design -which focused on the use of the database as a browser for the repository of a completed data set ---to be one that also functions as a project management support tool for planning the completion of the remaining half of the coastline scheduled for inventory. 


\section{Chapter 3 - Systems Analysis and Design}

Fundamentally the CBI database and interface project goal was to create a way to store and interact with data collected during field observations. Because the type of data collected is created by the specifications of the protocol, the database structure is guided by the way the data is conceptualized. The different types of data included digital imagery, digital facsimiles of the original field data, reference data, supporting protocol documents, numeric data, text data, geospatial and location information and connections to external data sets. The motivation to collect the data is noted in the background. This section provides examples of the tasks that the Coastal Biophysical Inventory supports in order to assist the management of intertidal resources. The Microsoft Access format database file required for this project is a table structure, an interface, and a programming environment. The database structure and the system design required a solution that integrated in that Microsoft Access environment.

\subsection{Problem Statement}

The problem to solve was to create a relational database and interface software that could be freely distributed to store an existing variety of observation data and could be supported with the standard suite of software installed on National Park Service computers in the year 2003 so that the data could be entered, reviewed and queried -including spatial query.

\subsection{Requirements Analysis}

The Requirements Analysis included summarizing the user needs for the system, the constraints upon system design, and addressing the five major components of a GIS for a system design that integrated tabular data, GIS data and an interface. This section will address the functional and non functional requirements derived from the user need and system constraints to apply to the system design.

\subsubsection{User Needs}

The user needs were defined by:

1. identifying the users,

2. relating the expected use of the system by the identified users and

3. reviewing scenarios of use.

This can be best summarized in the tables and a diagram on the following pages. 
Table 1. User Needs Summary of Examples

\begin{tabular}{|c|c|c|}
\hline Expected Use & User & Scenario \\
\hline $\begin{array}{l}\text { Input Tabular } \\
\text { Data }\end{array}$ & Bio-technician & $\begin{array}{l}\text { The bio-technician has a completed field } \\
\text { data sheet in paper form that needs to be } \\
\text { transcribed from paper to a digital } \\
\text { repository. }\end{array}$ \\
\hline Browse Data & Biologist & $\begin{array}{l}\text { The biologist wants to browse the data to } \\
\text { hypothesize any patterns between the many } \\
\text { aspects of the data that are impossible to } \\
\text { view on the data sheet alone. The biologist } \\
\text { will compare substrate, biology and photos } \\
\text { to formulate a query around. }\end{array}$ \\
\hline Query Data & Biologist & $\begin{array}{l}\text { The biologist will compare diversity of } \\
\text { species to substrate type to length of } \\
\text { coastline observed. }\end{array}$ \\
\hline Correct Data & Biologist & $\begin{array}{l}\text { The biologist will find errors entered on the } \\
\text { original datasheet and review them for } \\
\text { change or the biologist might notice conflicts } \\
\text { between what is entered in the database and } \\
\text { what the datasheet or protocol indicates. }\end{array}$ \\
\hline Browse Data & $\begin{array}{l}\text { Biologist - } \\
\text { Educator }\end{array}$ & $\begin{array}{l}\text { A marine educator is planning a series of } \\
\text { field observations of organisms and wants to } \\
\text { minimize impact on one spot with a large } \\
\text { group by visiting different intertidal areas of } \\
\text { high diversity that are easiest to access. }\end{array}$ \\
\hline $\begin{array}{l}\text { Statistical } \\
\text { Analysis }\end{array}$ & $\begin{array}{l}\text { GIS Data } \\
\text { Manager }\end{array}$ & $\begin{array}{l}\text { An estimated } 450 \text { segments means that } \\
\text { deriving patterns from the multiple attributes } \\
\text { will require analysis of the variation of } \\
\text { relationships of the data to itself. }\end{array}$ \\
\hline $\begin{array}{l}\text { Enter } \\
\text { Geographic } \\
\text { Data }\end{array}$ & $\begin{array}{l}\text { GIS Data } \\
\text { Manager }\end{array}$ & $\begin{array}{l}\text { The GIS user will have coordinates collected } \\
\text { via GPS in the field and will need to enter } \\
\text { them to provide the framework over which } \\
\text { the attribute data drapes. }\end{array}$ \\
\hline $\begin{array}{l}\text { Input } \\
\text { Geographic } \\
\text { Data }\end{array}$ & $\begin{array}{l}\text { GIS Data } \\
\text { Manager }\end{array}$ & $\begin{array}{l}\text { After creating line segments from the GPS } \\
\text { coordinates, the GIS data manager updates } \\
\text { the list of available Segments to add } \\
\text { observation records to in the tabular data } \\
\text { based database. }\end{array}$ \\
\hline
\end{tabular}




\begin{tabular}{|c|c|c|}
\hline Expected Use & User & Scenario \\
\hline Querying Data & $\begin{array}{l}\text { Resource } \\
\text { Manager }\end{array}$ & $\begin{array}{l}\text { An oil spill has occurred. The resource } \\
\text { manager wants to know which segments } \\
\text { were observed using the remote observation } \\
\text { protocol, indicating that accessing them is } \\
\text { extremely difficult and makes some cleanup } \\
\text { efforts impossible. The possible goal is to } \\
\text { prioritize oil spill containment boom } \\
\text { placement around these areas. }\end{array}$ \\
\hline $\begin{array}{l}\text { Analyze } \\
\text { Geographic } \\
\text { data }\end{array}$ & $\begin{array}{l}\text { Resource } \\
\text { Manager }\end{array}$ & $\begin{array}{l}\text { Make a count of the diversity of species for } \\
\text { each segment and rank the segments by this } \\
\text { count. Take the resulting query and join it to } \\
\text { the segment spatial file and symbolize the } \\
\text { range of diversity with a ramped color to } \\
\text { show high to low. Compare resulting map to } \\
\text { the area of a hypothetical oil spill. } \\
\text { Summarize the percentage of highly diverse } \\
\text { areas that would be affected by selecting the } \\
\text { segments that intersect the hypothetical oil } \\
\text { spill. }\end{array}$ \\
\hline Query Data & $\begin{array}{l}\text { Resource } \\
\text { Manager }\end{array}$ & $\begin{array}{l}\text { Researcher is studying organisms that prefer } \\
\text { beaches with pebbles as habitat. Select all } \\
\text { segments where the observation noted } \\
\text { pebbles for the substrate. Prioritize research } \\
\text { to beaches where pebbles are the primary } \\
\text { substrate or boulders are the primary } \\
\text { substrate and pebbles are the secondary } \\
\text { substrate. Review photos and USGS maps } \\
\text { for access to these locations. Create a permit } \\
\text { request to do research on the coastline } \\
\text { identified as optimum survey sites. }\end{array}$ \\
\hline $\begin{array}{l}\text { Maintain } \\
\text { Database, } \\
\text { Table } \\
\text { management }\end{array}$ & $\begin{array}{l}\text { Database } \\
\text { Manager }\end{array}$ & $\begin{array}{l}\text { The database manager may realize that there } \\
\text { are normalizations or de-normalizations of } \\
\text { the data that will improve efficiency and fix } \\
\text { table structure or relationships. }\end{array}$ \\
\hline $\begin{array}{l}\text { Maintain } \\
\text { Database, } \\
\text { Code } \\
\text { Management }\end{array}$ & $\begin{array}{l}\text { Database } \\
\text { Manager }\end{array}$ & $\begin{array}{l}\text { There are often repairs to programmed code. } \\
\text { The database manager may make } \\
\text { improvements to the database through code } \\
\text { in order to improve the performance of the } \\
\text { system or the interface for other users to the } \\
\text { system. }\end{array}$ \\
\hline
\end{tabular}




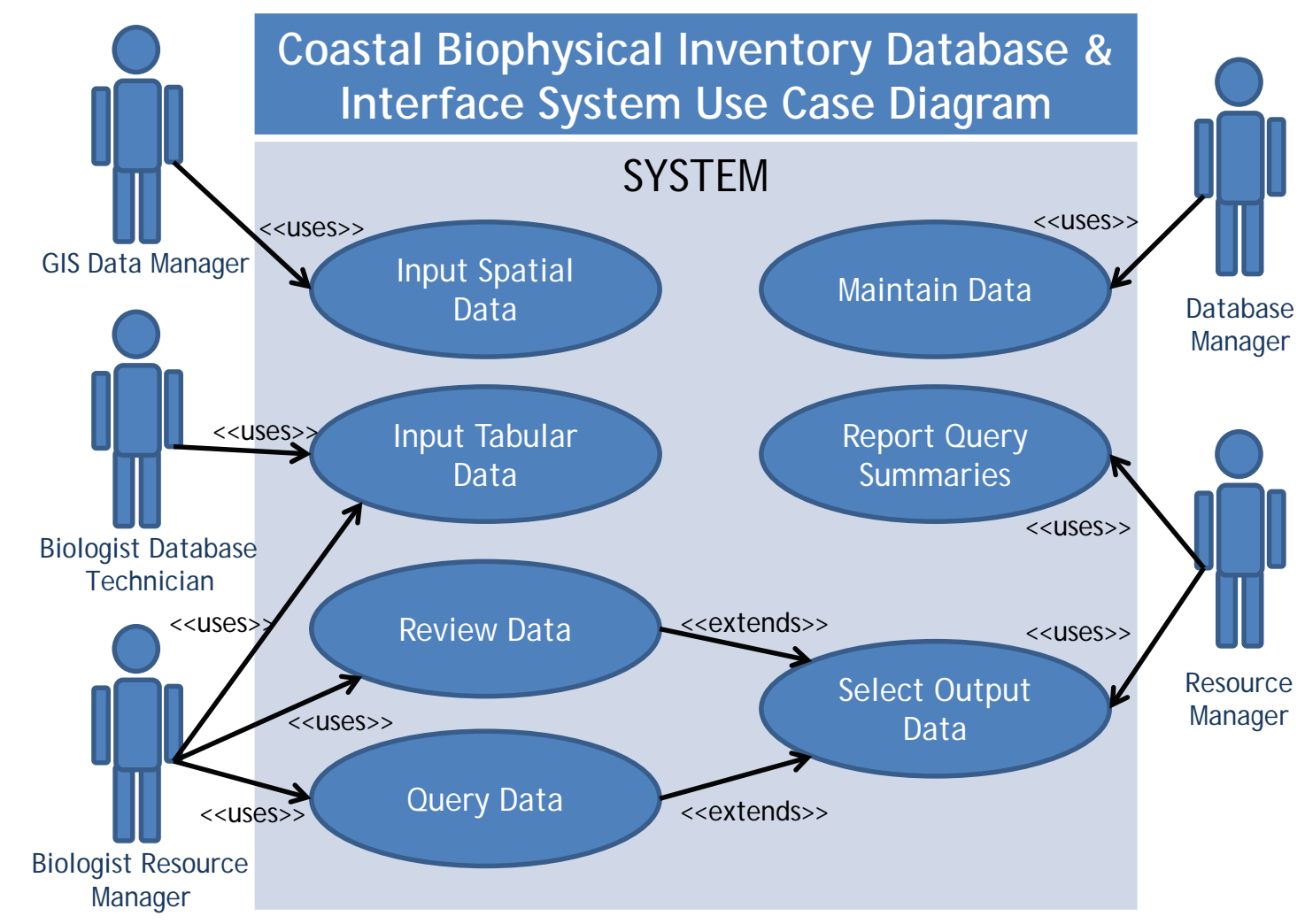

Figure 4. General Use Case Diagram

The primary users modeled in figure 4 are:

- GIS Data Manager, a user who will collect spatial data in the field and convert it to formats usable by the system for relating observation data to location.

- Database Manager, a user who will maintain the proper functioning of the database or its expansion.

- Biologist Database Technician, a user who will transfer field observations into the database.

- Biologist Resource Manager, a coastal resource manager (or equivalent at another agency) who will perform observations, interact with the system to input, correct, browse or query data and report summaries for decision making.

- Biologist, a user who will be engaged in the ongoing collection and use of the data, integral to maintaining quality of data and was the client's primary main use.

The examples provided are not exhaustive, but demonstrate the fundamental uses that the system should be capable of supporting. The interfaces with the system need be created through graphical user interfaces or workflows that allow interaction with the system. As outlined in the General Use Case Diagram in figure 4, the system will receive human interaction through the following interfaces: Input Data, Browse Data, Query 
Data, Select Output Data, and Report Query Summaries. For the system these interfaces are defined as so:

- Input Data: The "input data" interface includes the ability to enter tabular data directly into the Access Database. Accessible from the form will be tools for labeling and saving imagery data into a hierarchical folder structure and updating of Access Database with imagery file names and a foreign key to relate the stored image to a field observation record. Workflows for converting paper documents into PDF scans so that the electronic record of the raw data document can be related to the observation record similar to the field imagery. Methods for updating protocol records for quick reference. A workflow for updating spatial data using GPS measurements collected in the intertidal zone to delineate coastline inventoried and related to observation records.

- Browse, Query, Select Data: Data can be queried, browsed and selected through the tabular data interface or the spatial data interface, both of which are linked to limit the records displayed for what is selected in the other. The user will be able to view related images of site form air and ground surveys, display rectified aerial image in map display, query spatial data, browse spatial data, select spatial data, query tabular data, browse tabular data and select tabular data.

- Output Selection: To aid further analysis or respond to requests for summarized data, the data selection can be exported for use elsewhere in a relational database or GIS.

- Maintenance: The database will need repair or augmentation based on unidentified conflicts and changes in user needs. The database will have models and definitions that will enable some minor internal repairs.

The interfaces were envisioned to support the use of the system and these designs were part of the software's functional requirements. However, there is a large difference between creating a system that satisfies the use cases programmatically and one that remembers that its users are human and have emotional aspects to their use. This constraint or specification to use is often considered as an afterthought because it is often seen as being difficult to quantify or measure. Requiring that the interface must be pleasing to the user as a condition of proper functioning will assist in setting a higher expectation to the quality of the user experience which can lead to improved interface design. 


\subsubsection{System constraints}

The system was constrained from the beginning by

- the security protocols limiting users and hardware,

- the budget for software development and licensing,

- existing data type and format

- programming expertise for implementation \& maintenance

- data collection protocol

\section{Security protocols}

The National Park Service requires that employees and contractors that use federal computers that are part of the NPS network and its parent agency the Department of the Interior complete a security background investigation and ongoing security training. There are exceptions to this and they are documents and resources published to the public on the internet servers and File Transfer Protocol (FTP) servers that are password protected for transferring large data sets between partnering organizations. Background checks were not possible or appropriate for all users. This meant that data would need to be transferred on portable storage media or over password protected servers, and this required assessment of portable media to transfer data when network connectivity was not allowed or available to all users.

\section{Budget}

No additional funds for licensing or software purchase were budgeted. A clear goal was proceed with existing license agreements. Funds for additional programmer developer components were secured to resolve imagery display problems.

Licenses for Microsoft Office XP Professional (which includes Access XP/2002) already existed for all National Park Service computers. For those reasons, the creation of a system that incorporated those database and programming components would be functionally "free" to use within the National Park Service and satisfied budget limitations and avoided additional ongoing maintenance tasks. Computer systems at the California Department of Fish and Game Oil Spill Prevention and Response offices had similar enough operating systems and existing licenses for Microsoft Office Professional with Access and ArcGIS that distribution to that agency would mean that distribution of the software and database there would be unencumbered by additional license and royalty fees.

\section{Existing Data}

The observation data collected before 2007 was stored in a Microsoft Access 97 format relational database. The spatial data were stored as line features in an ESRI Shapefile format file. Images that documented the locations were stored as jpeg files and field data sheets were stored on paper. The existing database contained the observation records collected on the paper field data sheets, but had not been checked for error against the paper data. The existing user interface built into the Access database file incorporated the 
ability to enter data, review data and spatially query the data; however it was broken due to the incomplete adaptation of the Glacier Bay National Park and Preserve's

CoastWalker database to the protocol revised for California's coastline. Because it was broken it could not link to or make use of the existing spatial data and this was where the client sought assistance.

\section{Programming expertise for implementation \& maintenance}

Two separate constraints for programming experience were part of the requirements for the project. Sufficient skill to organize and program the system interface with available software and the skills of the client to use and maintain the system delivered.

Online distribution of the database was a desirable alternative for low licensing costs and ease of sharing data; however, the available map hosting environment at the time was ArcIMS. Previous experience with the implementation and management of the ArcIMS environment made it an undesirable option for hosting on the National Park Service servers.

Programming expertise needed to match available time for project. Application development (user interface and database) was estimated at approximately 3 months based on the use of Visual Basic 6 or Visual Basic for Applications programming language. In 2003, Visual Basic 6 (VB6) was a robust programming environment, with many programming references and online user communities for professional assistance. Compared to other programming languages that could use Map Objects ActiveX objects for creating a custom map interface, Visual Basic 6 ranked higher as a choice for a new programmer for a number of reasons most important was the clear documentation of the object models and the built in "garbage collection" of VB 6 compared to the need for more explicit programming of memory management in other programming languages.

Other languages, ActiveX programming objects and programming software were investigated including the languages Visual Basic .NET, C++, C\#, Java and Delphi, and the objects MapObjects Java, MapObjects Windows Edition, and ArcObjects. A paucity of examples in programming languages other than VB 6 or VBA made them unlikely candidates for the programming environment for the rapid development of the database interface project for a new programmer. The initial client for the project (David Schirokauer, biologist and GIS manager at Point Reyes National Seashore) noted that other programming environments outside of those built to use Microsoft Office XP or MapObjects LT 2.0 would be difficult to support with the programming experience at the various National Parks that might use this database for their inventory efforts.

\section{Data collection protocol}

The existence of a protocol already in use meant that the interface and database design derived from the protocol and design had little reciprocal influence on the revision of the protocol. Any changes or functioning of the system that required changes or adaptations to the protocol would be undesirable and costly in effort. 


\subsubsection{Functional and Non Functional Requirements Summary}

Within the constraints to the system described above, the database and interface was designed to support the interaction with observation data for management of the resources. This required an analysis of the intended uses and resulted in an understanding of the functional and non functional requirements. The requirements investigated in the previous two parts of the Requirements Analysis section are summarized in the following table.

\begin{tabular}{|c|c|}
\hline Functional Requirements & Non Functional Requirements \\
\hline $\begin{array}{l}\text { - Store data, including tabular data, } \\
\text { imagery data, documents, spatial } \\
\text { data } \\
\text { - Review data through browsing } \\
\text { tools in a custom interface and } \\
\text { other software } \\
\text { - Analyze data via query and } \\
\text { summary tools in a custom } \\
\text { interface or by compatibility to } \\
\text { other GIS or relational database } \\
\text { management software. }\end{array}$ & 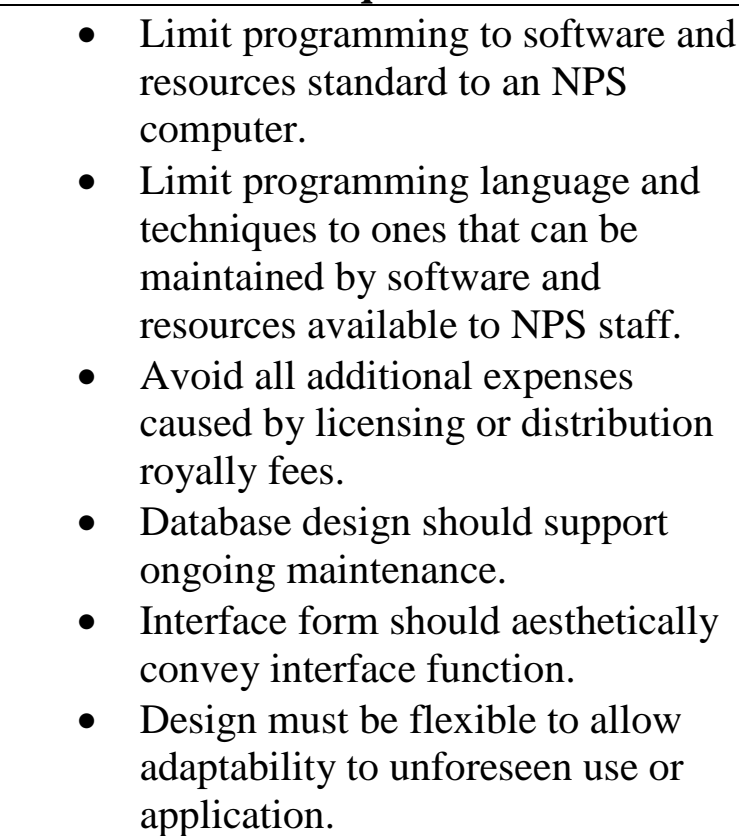 \\
\hline
\end{tabular}

\subsection{System Design}

In 1896 the architect and designer Louis Sullivan coined the phrase "Form ever follows function" (Sullivan 1979) to describe the culmination of architectural design history and the creation of a new type of tall building (called skyscrapers today) that were built using the new building materials of steel, iron and reinforced concrete that incorporated elevators, water, electricity, telecommunications and air circulation. This phrase has influenced all modern designers who have come since and is the pragmatic aesthetic for the priorities of designing complex objects that are a system. System design for buildings is analogous to system architecture in the world of geographic information systems and the field borrows heavily from the lexicon of building architecture. Included in that aesthetic is an expectation of elegance revealed by design that optimizes the function to a form so efficient that it evokes an emotional response to beauty. When constrained by resources, system design which leads to interface design, often stops when it functions, stripping the original aesthetic intent of the idea of form following function. Systems built from modular components function and allow the rapid development of customized software and GIS applications, but they also often lack an integrated and efficient 
elegance because the design stopped at "good enough" after piecing the functioning parts of the system together.

Within the context of the limitations of the programming environment, licensing constraints, the client's ability to maintain the system, existing examples, expected uses, limited application time, and the structure of the data already collected, a system that could accomplish the clients goals evolved. At all times there was an expectation that the system interface not only functions correctly but that it also facilitates easing the users experience and reducing unnecessary effort. A graphically inoffensive design and systematic logical structure was included as an aesthetic function.

The system design for this project was essentially for a geographic information system. The most effective way to organize conceptualizing geographic information system design is to decompose it into its five major components of

1. people,

2. software,

3. hardware,

4. methods and

5. data.

The next step was to identity the functions to satisfy the requirements in each component. After that the design reassembled the components as a system and then modified the design iteratively through feedback and use changes. The next part of this section addresses each component to identify the required features.

\subsubsection{People}

Most of the people, roles and uses have been discussed already in the background and the use cases because the use by people sets many of the system functional goals and design constraints. Because of their importance in the system design, the system users that were essential to describe the system's scope of functions were defined by the tasks set out in the protocol and were defined as:

1. GIS manager: the GIS manager organizes and creates spatial data. Maintains standards of functionality and metadata, assists biologists with spatial queries of observation data. Manages equipment and network that system will use. Manages GPS equipment and workflow for collecting data and bringing into the system. The GIS manager works with the resource manager to interact with spatial data and construct reports that map the results of queries.

2. Field ecologist: the field ecologist makes the observations in the field of the parameters of the ecosystem identified in the observation protocol. This includes identification of organisms observed, characterization of the substrate, and characterization of the intertidal zone, observation conditions, and photo documentation of each site visited. The field ecologist estimates the breaks or changes in type of intertidal ecosystem by the substrate type, which defines the spatial organization of the attribute data collected during the observation of a location. The field ecologist determines the location of the biology transect that will be mapped and works with the mapping technician to establish and document the transect location. The field ecologist works as a team with the mapping technician and needs to be able to assume their role. The ability for the 
individuals to exchange roles assists with troubleshooting in the field and to allow occasional relief from the physical strain of crawling on algae covered rocks during identification of organisms close to the surf by switching roles. The field ecologist records observations to a data sheet.

3. Database technician: usually the role of the database technician is completed by the same person who is the field ecologist to assist with catching gross error and resolving handwriting legibility problems of field data sheets that record observations. The database technician enters observation data recorded on the data sheet. The database technician sorts, labels and organizes digital imagery from the field observations into the CBI folder structure on the digital storage media. With the direction of the database manager, the database technician scans the data sheets into electronic documents and labels and organizes the documents on the digital storage media by the segment number for that observation.

4. Database manager: The database manager is usually an extension of the responsibilities of the GIS manager. This person organizes the observation data and programs the interface. The database manager works closely with the biologist to create domains of reference that can be used for improving data accuracy and with the GIS manager to ensure that the essential linking between observations and location data work. The database manager creates the software structure, hierarchical organization of storage on media, and database interfaces. With the other people using the system, the database manager creates workflow efficiencies and provides maintenance support, error correction, and assists in requests for distribution of the database. The database manager works with the resource manager to facilitate interface with the database, providing user instructions and assisting with structuring inquiries if the resource manager is unfamiliar with the database.

5. Mapping technician: the mapping technician is often also a field ecologist to provide a minimum of two people in the field to collect data with and efficient team size and in the interest of safety in the field. This person works with the GIS manager for planning field work locations based on the logistics of access to the sites, the window of opportunity for research based on tide height and time, meeting expected precision based on GPS satellite configuration at time of tide, and inputting coordinates for navigation to where previous and adjacent inventory work had ended. The mapping technician records the boundaries to the coastline section (called a "segment" in the CBI) and the biology transect endpoints. In the field this person enters attribute data and maintains the mapping standards of the protocol. Part of the GPS to GIS workflow, the mapping technician establishes the segment number in the field for the observation record and assists the GIS manager with transferring data contained in the GPS memory system to the GIS for processing further.

6. Resource manager: The resource manager has the most variable definition, but primarily is one of inquiry in order to get a sense the intertidal resources by reviewing the recorded observations and generating summaries and reports of data to create tabular reports or maps. The resource manager may use this to prioritize additional research at specific locations or to assess changes between observations at the location. The resource manager includes National Park Service staff that 
are part of the Natural Resource Damage Assessment team and process after and oil spill.

\subsubsection{Software}

This section will cover the software that will be part of the system, the software used in the project, the software used to program software and the software components used to create custom software. The system software components are presented in order from the general operating environment to the specific modules of software code or objects.

\subsubsection{Base system software and Operating System environment}

The National Park Service have software license and networks that limit the use of software to specific versions and releases in order to coordinate efficiencies for information technology management agency wide. The operating system software will be an important constraint on hardware and the software options available. With the exception of a handful of machines, Microsoft Windows XP Profession was the accepted and supported operating system for the National Park Service from 2003 to mid 2011. Microsoft Windows 7 Professional is the anticipated replacement. Microsoft Office 97, XP, 2003 and 2007 were an evolving system of options for managing the project with Microsoft Word, Excel, PowerPoint, Visio, Project, Outlook and Access being used as essential to the project management, reporting, document management, email communication and design.

\subsubsection{Software essential to database and interface function}

Critical to the project was Microsoft Access. Microsoft Access files were the most complete and supported solution for database management and the existing format with the most complete examples to emulate. Migration of the database to newer formats was anticipated, expected and implemented. Within the Microsoft Windows operating system there are programs that are installed by default such as Internet Explorer, Notepad, and Windows Photo Viewer or Windows Picture and Fax Viewer. These would fill a critical role of the project as supporting programs to completer, but because of their appearance on all NPS computers, they would be considered for ways to become functional parts of the system rather than interstitial support for tasks. The inclusion of those three software tools on every previous release of a Microsoft Windows Operating system after Windows 95 indicated that the risk to the system would be small if it relied upon them without control over their production or life cycle and could greatly improve development time to use them in the system as is.

\subsubsection{Software for geospatial information management}

ESRI's ArcGIS (ArcMap, ArcCatalog, and ArcToolbox) with an ArcInfo level license was used as the primary spatial data editing, data management and metadata management tool. The use of other spatial data and metadata management software in place at the NPS was avoided for this project because of the desired portability afforded by the XML format FGDC compliant metadata file created natively by ArcCatalog which was later 
supported by and used in the NPS Metadata Tools Editor to assist with NPS metadata standards. ERDAS Imagine software was used for the correction and conversion of aerial imagery. The following extension tools to ArcGIS were used to improve editing workflow and data management: ESRI Spatial Analyst extension for raster analysis tasks, DataEast XTools Pro for attribute table calculations of area and length, ET

SpatialTechniques GeoTools for tools that automate the reordering of polyline direction that are sometimes changed during editing of large when the line is split, and NGIS Australia Project Copier MXD for quickly packaging and sharing data and interim ArcGIS projects and for snapshot archiving of projects in progress.

\subsubsection{Software for GPS project management}

The software available for GPS project management with the National Park Service was Trimble Pathfinder Office and was used to manage the connections, data dictionary authoring for data entry forms on field data loggers, uploading waypoints for navigation, downloading coordinate data and attributes from data logger, and correction of coordinate data for improved accuracy. This allowed the use of three different models of available GPS devices because of the compatibility of Pathfinder Office with the software on those GPS units which included Trimble Asset Surveyor 5.27 and TerraSync 3.2 run on the Windows Mobile operating system.

\subsubsection{Programming tools}

Visual Studio 6.0 was chosen as the primary programming environment software which contained Visual Basic 6 (VB 6) programming language. Visual Basic 6 projects using MapObjects were plentiful and the language was designed for ease of use by new programmers. Additional database programming features such as Active Data Objects (ADO) control tools for programming database application interfaces were integrated into the software. Visual Studio 6 also programming included objects such as the DataRepeater object which allowed the creation of interfaces similar to the repeating forms available in Microsoft Access and this was seen as a powerful way to represent the complex sets of records that include photographs and the special arrangement of data entry fields for more efficient data entry or the display of results with a varying number of records. Layout of interface forms in Visual Studio 6 is also intuitive to use because it is similar to many of the layout tools in Microsoft Office that were already very familiar. Visual Studio 6 has a compiler integrated into it, allowing the Visual Basic code to be converted into a machine code executable file. The Visual Studio installation included software to make self installing software programs that register libraries and automate the installation of customized, compiled and executable software.

\subsubsection{Programming components for interface}

One of the clear deliverables is to construct a custom interface and database with spatial query tools. In addition to the controls common to the Visual Studio programming environment, special ActiveX controls were needed for creating the interface. Some controls were custom made and compiled and saved as ActiveX programming COM (.ocx file extension) objects or dynamic libraries (.dll extension) of code. ESRI 
MapObjects LT 2.0 were the software programming components used to create the map display interface that responds to interaction through event driven function and automated function.

\subsubsection{Hardware}

Many types of hardware were used in this project. With the exception of the GPS hardware, tools used during field observations are not in the scope of this document and their use can be found in the inventory and mapping protocol. Data transfer between sites was a major part of the workflow expected, so the hardware to facilitate that is discussed below. Minimum computer hardware to manage the project and operate the database and interface are summarizes below.

\section{Data Transfer}

Data transfer servers using file transfer protocol (FTP) were used to coordinate the exchange of project data via a NPS server between users without federal computer network permissions. A high speed network system with internet access was required. Network data transfer limits at different user locations along with security restrictions to network access required alternative data transfer devices.

Hard drive or flash media were the preferred transfer hardware. DVD and CD media, while superior in portability and cost, were an expensive workflow due to slow transfer time compared to the transfer speed of USB 2.0 speed digital storage media peripherals and the additional time required to remove write restrictions to every file after the project or data is transferred from read only media to hard drive media before use. Through compression of electronic files and the removal of files that were archives of versions of the project in development, the size of the completed project for publication was estimated for less than 8 gigabytes and became a specification for the final deliverable in order to make use of Dual Layer DVD writing technology for relatively inexpensive media (approximately \$1.20 per 8.5 GB Dual Layer blank media) and shipping expenses. Therefore a Dual Layer DVD drive was required for the reporting functions of the database manager as part of the system.

\section{Computer minimum specifications}

With the exception of the GIS Manager, the computer hardware used in the project was constrained by the software used in the project, operating systems of the users are constrained by the installation and the resulting customized database interface software with its supporting tabular, imagery and spatial data, will have a common denominator of computational ability, storage capacity, input and display. Because of limits to purchasing computer hardware by contracts in place at the federal government that prioritize computer components manufactured or assembled in the United States of America, DELL branded machines running 32 bit processors were the default choice for computers for this system by a majority of the users. Exceptions to this limitation were available to the GIS Manager to purchase computer equipment optimized to support GIS 
work. The minimum requirements expected for the general user and the Coastal Biophysical Inventory Database were as follows:

- 104 key keyboard, US English

- Mouse or pointing human interface device

- Pentium 4, 2 GHz processor

- 2 gigabytes of RAM

- 200 gigabyte hard drive

- 1280 x 1024 resolution or greater monitor, landscape format

- Graphic processor capable of displaying 32 bit color space at the minimum monitor resolution.

- Unused USB 2.0 port, not on a hub.

\section{GPS}

Protocol development for the mapping of substrate in the GLBA CoastWalker project identified 20 meters as a pragmatic threshold for a minimum length of coast to be inventoried as a discrete unit. Many GPS units in use at NPS could obtain coordinates for location to within 3 meters of accuracy. This accuracy seemed incompatible with the 20 meter minimum unit for coast line. If a coastal section measured was 20 meters, for example a bedrock outcropping between two sandy beaches, it meant that there was reasonable expectation that the length of the coastline measured could actually represent as little as 14 meters to as much as 26 meters when accounting for the range of error. For this reason GPS devices that were capable of measuring location with real-time corrections for navigation within 1 meter of accuracy, creating an expectation that the smallest segment of coastline measured could reasonably be expected to be as short as 18 or as long as 22 meters.

The devices available that could make measurements within 1 meter were limited and additional specifications would narrow those as choices. High cliffs that obscure satellites, coastal hills, and headlands could often obscure the line of sight reception of signal from spaced based references for differential correction (e.g. WAAS or SBAS geostationary satellite systems). Because ground based radio waves propagate around obstructions differently at lower frequencies, GPS devices that were capable of receiving and using Radio Technical Commission for Maritime Services (RTCM) 104 serial protocol from continuously operating differential-GPS reference stations was an essential hardware requirement over space based references in order to correct real time coordinate accuracy to within one meter.

Additional operational needs for long field time in environments inhospitable to electronic device required additional equipment specifications. Battery life of 8 hours was needed for observation trips that required hiking in and camping the evening before a morning low tide in remote locations. Exposure to salt water and sand is unkind to electronic equipment, therefore ruggedized or splash proof devices were needed. The following GPS units that met the hardware constraints were available for use from other programs within the National Park Service and were borrowed for the project:

- Trimble Pro XR

- Trimble GeoXT with Beacon on Belt receiver

- Trimble GeoXH 
The hardware described above represented the scope of devices used to collect, store, convert, correct, program, manage, analyze and display the observations and custom interfaces of the Coastal Biophysical inventory project.

\subsubsection{Methods}

The methods used for the completion of the project and their documentation were a significant part of the project's work and a critical part of the change in scope. Because the methods were expected to be repeated and used by others, attention to the quality of description was essential. There were many methods created and documented. Those documents were detailed and are part of the distribution of the software and the names of the documents will be referenced in this section only instead of presented in their entire form here or as an appendix. All documents presented here are part of the digital data collected for this document and can be found in the "Protocol" directory of the data set or linked through the "Documentation” page of the database user interface.

\section{Programming}

Methods of programming were to follow object oriented programming standard to and part of the Visual Studio 6 programming environment. Hungarian Notation (Simonyi 1999) was the method of keeping code more organized, clear in functionality, and it assisted legibility. Object and variable naming in VBA followed the Stan Leszynski and Greg Reddick VBA naming conventions derived from Hungarian Notation.

Programming software usually followed a technique of adaptation and experimentation. Examples of functional goals were often described in pseudo code, an informal method of describing functions and results in natural language. Examples of analogous solutions were sought first in the programming language VB 6 to be deconstructed and adapted for new function. Some code examples were extracted as modules and use unchanged with the citation for their origin embedded as captioned code.

Reference manuals (Microsoft 1998) and example code were the foundation for all work done. The robust VB6, Access VBA and MapObjects programming communities represented in virtual forums (Tecumseh Group n.d.) (UtterAccess n.d.) (ESRI n.d.) (Experts Exchange 1996-2009) on the internet and the archived conversations there were essential for resolving faulty programming logic and providing alternate perspectives that resulted in quick solutions. On rare occasions, some programming objects and techniques were so sparsely documented by the manufacturers that examples of their use in these programming discussion forums was the only point of reference

The final method used was iterative design. A mock up of the software interface and the underlying data structure was designed. A GIS browser interface piloting example software was programmed to work out fundamental design goals to satisfy the use expected. It was also used as a way to evaluate programming skills and evaluate estimates of project time. A full version of the project was attempted, and the majority of it completed but eventually abandoned because it failed to support a change in the emphasis of use. Lessons from that attempt were used to rapidly develop the final software interface and GIS design. 


\section{Data Management}

Data management was an extensive and time consuming endeavor to compile, convert and organize into base reference layers. Data were managed to support cartographic visualization in an interface, reference information for overlay comparison, and relation to the numerous field-observation records. A hierarchy of folders used for ongoing project work and interstitial files in progress was created and in this was the programming "sandbox" where results could be created and tested separate from another set of folders that represented the final completed project. Spatial data were "quarantined" until it could be processed or converted and given complete metadata according to standards and workflow guidelines developed for the project.

The many tasks of data management were improved by developing a series of instructions to repeat for consistency and ease of processing. A documented workflow of methods for the management of GPS data can be found in the supporting project document "Methods for processing Coastal Biophysical Inventory (CBI) field coordinates into line features of the coast” (J. Kinyon 2008). See Appendix F for the full document. Metadata standards and methods for improved workflow through the efficient use of XML templates was created to improve metadata documentation and can be found in the supporting document "Metadata Standards and Guidelines for the Pacific Coast Science and Learning Center" (Goodman and Kinyon 2006). This document can be found in the "Protocol" directory of the data set or linked through the "Documentation" page of the database user interface.

\section{Documentation of methods}

The documentation of the database interface project was a method of both project management success and was the most unanticipated and time consuming expansion of scope when the project needed additional documentation of methods of people interfacing with the system. The creation and improvement of these methods account for approximately 3 of the 5 years spent on this project. It is beyond the scope of this document to discuss the separate work to complete all the documentation that was essential to complete for proper function of the system. They will be summarized here and are provide as supporting documents on the DVD submitted with this document.

Documentation of data management was required. Metadata for data acquired or created was required with an addition stipulation that this project component that would not compromised for other goals. Documentation of workflow or best practices to enable ongoing maintenance was part of the project plan and included into some metadata documents for the description of data processing.

Documentation of data collection methods were not originally part of the system design because the existing data drove the emphasis of the design to be a system for data review. Documentation of collection methods became part of the design requirements when the emphasis of design shifted to one of data entry when management of the project changed hands. Logs and notes about the effort of collection methods were used to be able to estimate time to complete remaining work in the field and were metadata about the field data sheets. 
Additional documentation of the way the GIS database was designed were needed for the GIS Manager and proved useful to the Database Manager for eliminating programming goals for the MapObjects based interface that became obsolete Techniques for the successful connection of Microsoft Access tabular data to ArcGIS needed to streamline training of use of the database and GIS software with examples of how to make "Join" and "Relate" connections within ArcGIS Additional guidelines were needed for the regular task of spatially representing many to one relationships in tabular observation data. "Relating Access to ArcGIS" (J. Kinyon 2009) illustrates the suggested methods to facilitate that analysis and is included as a supporting electronic document accessed through the interface.

\section{Database interface}

Methods of design to improve data entry included goals of efficiency for both the interface and the field data sheets. A method of parallel and integrated design was used throughout the iterative design of the electronic and paper data entry process. It was important to relate the most efficient workflow into the protocol for data collection. The layout of the field data sheet reflects that efficient workflow outlined in the revised inventory and mapping protocol and the data sheet had a separate iterative design based on feedback from field use so that the layout reflected and improved the data collection process. In tandem, the user interface for data entry was designed to proceed through the work flow in the most efficient way for data entry but also have a logical structure that emulated the field data sheet and was analogous to the sequential reading of sections of the paper field data sheets. The Microsoft PowerPoint presentation "Coastal Biophysical Inventory 2.3 Introduction to Data Entry and Explanation of User Interface and Tools” (J. Kinyon 2009) is a manual that reflects the method of design integration of different complementary data entry methods by showing the suggested workflow for entering paper data into the database user interface.

\subsubsection{Data types}

The system design anticipated that data will be collected, corrected, stored, viewed, analyzed and shared using the database and interface after completion. Data is intended to be public access and security constraints such as password protection are not required. The Microsoft Access relational database has functional limitations of approximately 1.5 gigabytes of storage within the Access format database. This limit kept some data types from being organized and stored inside a relational database file. A quick characterization of the data used in the system including examples of use and management plan associated with each type of data is summarized in Table 2 below. 
Table 2. Data types, their anticipated uses and management for system design

\begin{tabular}{|c|c|c|}
\hline Data type & Example of use & Management \\
\hline $\begin{array}{l}\text { Non spatial } \\
\text { raster }\end{array}$ & $\begin{array}{l}\text { field photos, approximately } \\
\text { 1800, graphic and logos }\end{array}$ & $\begin{array}{l}\text { Stored in groupings related to content } \\
\text { in logically organized folders with the } \\
\text { project. Related attributes in tables. }\end{array}$ \\
\hline Spatial raster & $\begin{array}{l}\text { aerial Imagery, nautical } \\
\text { charts, elevation grids, } \\
\text { topographical maps, }\end{array}$ & $\begin{array}{l}\text { Stored in groupings related to content } \\
\text { in logically organized folders, to the } \\
\text { project. Exceeds file size limits of } \\
\text { Personal Geodatabases. Organized } \\
\text { through raster catalogs. Metadata } \\
\text { stored as XML format document. }\end{array}$ \\
\hline Spatial Vector & $\begin{array}{l}\text { Coastal Biophysical } \\
\text { Inventory Segments, Raster } \\
\text { Index, Boundary, Google } \\
\text { Earth files. Overlay and } \\
\text { query analysis with results } \\
\text { reported in subset of vector } \\
\text { data files. }\end{array}$ & $\begin{array}{l}\text { Stored in groupings related to content } \\
\text { in logically organized folders with the } \\
\text { project. Stored in Personal } \\
\text { Geodatabase. Connected to ArcGIS } \\
\text { documents and tabular data } \\
\text { programmatically, alteration of } \\
\text { location breaks function. Metadata } \\
\text { stored as XML document }\end{array}$ \\
\hline $\begin{array}{l}\text { Map } \\
\text { documents }\end{array}$ & $\begin{array}{l}\text { project management, } \\
\text { analysis }\end{array}$ & $\begin{array}{l}\text { Relative linking to spatial data, stored } \\
\text { in project folders by project name }\end{array}$ \\
\hline \multirow[t]{2}{*}{$\begin{array}{l}\text { Reference } \\
\text { documents }\end{array}$} & $\begin{array}{l}\text { Protocol, workflow and } \\
\text { method guidelines, manuals, } \\
\text { forms, data sheets, diagrams }\end{array}$ & $\begin{array}{l}\text { Version tracking, Stored in groupings } \\
\text { related to content in logically } \\
\text { organized folders with the project. } \\
\text { Available through data interface. }\end{array}$ \\
\hline & & $\begin{array}{l}\text { Email, shared access, use to track } \\
\text { milestones and as reference for data } \\
\text { sets. }\end{array}$ \\
\hline Software & $\begin{array}{l}\text { Installation files, executables, } \\
\text { tools and sample code }\end{array}$ & $\begin{array}{l}\text { Stored in groupings related to content } \\
\text { in logically organized folder with the } \\
\text { project. }\end{array}$ \\
\hline Metadata & $\begin{array}{l}\text { Document origin and } \\
\text { provenance of data. }\end{array}$ & $\begin{array}{l}\text { Stored as "side car" file adjacent to } \\
\text { data file it is referencing with the same } \\
\text { name and in XML format. Some } \\
\text { metadata stored in tables. FGDC } \\
\text { compliant NPS standards. }\end{array}$ \\
\hline
\end{tabular}




\begin{tabular}{|l|l|l|}
\hline Data type & Example of use & Management \\
\hline Spatial Tabular & $\begin{array}{l}\text { Waypoint files for } \\
\text { navigation, determination of } \\
\text { boundaries }\end{array}$ & $\begin{array}{l}\text { Corrected, transferred and stored } \\
\text { inside a GPS project management } \\
\text { system. Stored in original and } \\
\text { corrected format with the same name } \\
\text { by folder with date of field work used } \\
\text { as name. Converted to Vector data. }\end{array}$ \\
\hline Paper Records & Field data sheets & $\begin{array}{l}\text { Scanned into digital files and stored } \\
\text { with observations. Paper objects } \\
\text { organized alphabetically by coastline } \\
\text { Segment identifier in folders by event } \\
\text { name. Paper data sheets stamped with } \\
\text { a unique and sequential number linked } \\
\text { to database of observations. }\end{array}$ \\
\hline Tabular Data & $\begin{array}{l}\text { Observation records, file } \\
\text { pathways for link } \\
\text { management, Field logs, } \\
\text { Process monitoring, error } \\
\text { tracking }\end{array}$ & $\begin{array}{l}\text { Microsoft Access database, } \\
\text { occasionally stored as Microsoft Excel } \\
\text { worksheet for reporting }\end{array}$ \\
\hline
\end{tabular}

\subsection{Project Plan}

The project plan for the execution of this project included the following major tasks:

1. Delineate scope of work.

2. Change order process to document and manage scope and schedule.

3. Detailed IEEE formatted Software Requirements Specifications Document to guide functional requirements and programming goals.

4. Develop cartographic specifications for map interface and data browser

5. Software programming training plan

6. Pilot project to test soundness of project plan.

7. Testing schedule for iterative design and error analysis.

8. Access Database development

9. Develop project execution plan with SWOT assessment

10. Documentation of work

11. Create GIS comprised of tabular data, GIS data and graphic user interface.

\subsubsection{Delineate Scope of work}

Approximately sixty four hours of research, training and planning were devoted to each of the components of the project plan which was a thorough breakdown of the scope of work and strategy for completion. After five years since that plan was created in February of 2004, all those tasks still apply as defined. All of the tasks completed were instrumental in this project being completed-except the management of scope and schedule through a system of documenting change requests. I will address most of the major tasks listed. 


\subsubsection{Change order tools for project management}

Change order tools required that any change be documented to estimate change in work or effort planned and if that change could derail the project's completion. Change would not be implemented or was implemented if the risk of failure to project was significant and could not be deferred. This change order plan was between the client and me to keep programming on track to repair the existing database with a spatial browsing interface. A new change order process was not developed after the management of the project changed and made the change order process outlined in the original project management plan obsolete.

The response to the change in management dropped the change order process until software specifications could be derived from clarified data collection protocol. Emphasis on data entry for completion of project changed the interface and use specifications and prompted a cascading redesign of database, data specifications, data collection protocol. Change requests after the redesign were completed continued in two ways, error logs for database user indicating interface or data failure and feature requests based upon written documentation of use, suggestions for improvement, evaluation of benefits to improved data entry and estimated time to program new or revised features. Once overwhelming change to the project was stabilized into revised software specifications, the completion of the software required few changes.

\subsubsection{Software specifications planning}

Software specifications plans were made by following the methods and template (Wiegers 1999) of Software Requirements Specifications Document (IEEE 1998), It was time well spent to experience the systematic logic to tackling the large challenge with detailed inspection of all parts that the template and methods of analysis provided. The software specifications document provided the greatest guidance to the development of the project with the Project Management Plan. The following components of the software and the software environment were addressed and outlined in the Software Specifications Document:

- Software description

o Interfaces (System, hardware, software, user, and communications)

o Memory constraints

o Operations

o Adaptations to installation site

o General functions

o User characteristics

o Constraints on system

o Assumptions and dependencies for use

- Specific requirements

o Interface specifications (graphical, organizational, and programming specifications to support data and map interface for the system, hardware, software, user and communications)

o Functional Requirements for the user classes (Database Technician, Resource Manager, Database Manager and GIS Manager) 
o Performance specifications

o Logical database requirements

o Design constraints and standards compliance.

o System attributes (reliability, availability, security, maintainability, and portability)

- Change management process (identify, log, evaluate and updated the software specifications)

\subsubsection{Cartographic specifications}

Cartographic specifications were developed during the original planning for this project in order to guide the design and function of a map interface with rich tabular data. Many of the original interface design specifications persisted throughout, especially the specifications for the use of tabbed form controls to divide data tasks entry into logical sections (pages) for each observation record. Layout of individual tabbed pages makes it easier to navigate sections displayed on the limited area and resolution of a computer screen. Cartographic expectations assumed a screen resolution of approximately 100 pixels per inch. Thoughtful organization, function integrated with data collection method, and a palette expressed in a layout that is inoffensive were part of the non functional requirements. Mapping information were expected from a variety of sources and scales, however representing 1 to 24,000 scale data were made the priority of the specifications and reference data of a smaller scale were avoided.

\subsubsection{Software programming training plan}

Once the software specifications and constraints upon the system were understood, a plan to get instruction to complement existing skills to complete programming was made. Software programming training planning included instruction at the University of Redlands via MS GIS program faculty, one on one tutoring with adjunct faculty, instructor led courses through ESRI, instruction through online curriculum through ESRI, self directed study training using guided instructional manuals, participation in software programming discussion groups, and independent study that culminated in the completion of the pilot project "Hummingbirds of California." 


\subsubsection{Pilot Project}

A pilot project was needed to work out design details for implementing software specifications and evaluate the success of the software programming training. A pilot project was planned to demonstrate that the components and programming environment could actually complete the programming tasks envisioned. Completion of the pilot project allowed review of time expended in order to calibrate estimated time of completion.

\subsubsection{Access Database Development}

No plan for the development of the Access Database were made because the Access Database was already made and in use. However, a hypothetical redesign of the database structure was made to evaluate if any improvements or efficiencies could have been made. The foresight of the database redesign done as an experiment proved extremely useful later when the database had to be created new in order to align with the changed emphasis in use of the database interface to focus upon data entry.

\subsubsection{Testing Schedule}

A plan for testing database interface software occurred in two ways. First, the pilot project used a dataset of limited data which allowed ready understanding if the error encountered while testing was a product of the code or the underlying spatial or tabular data used. This created knowledge based on experience regarding when to expect or perceive error in the software function. The second testing schedule was for regular review of the interface by the potential user to evaluate if the interface was functioning and to log the unexpected use (or mis-use) that caused errors. The second testing schedule was not regular according to a calendar, instead versions were released to the different users and for each version data entry work was completed on the project until an error kept data entry or data error checking from continuing. Errors were documented and reported back via an error log as were feature requests or design suggestions. See Appendix $\mathrm{G}$ for an example of an error log communication between the user and the database manager.

\subsubsection{Project Execution Plan}

The project execution plan included a scheme of implementation so that the scope of work and major tasks followed sequential steps (Tomlinson 2003) summarized in a checklist of milestones for successful GIS planning (Pal 2000):

1. Conduct a Requirements Analysis

2. Develop a Pilot Project as proof of concept

3. Design logical and physical databases

4. Develop the mapping specification

5. Acquire quality reference data

6. Set up GIS organization

7. Acquire Hardware and Software, and train personnel 
8. Develop the data base design to support the identified applications

9. Develop applications from the prioritized list identified in the Requirements Analysis document

10. Develop procedures and standards for data conversion

11. Convert data to support applications being developed

12. Develop data maintenance policies and procedures

13. Develop applications for other users and increase the user base over time

Each action or change in the direction of project was evaluated for the strengths, weaknesses, opportunities and threats or risks (SWOT analysis) to the completion of the interface design. When the responsibility to manage the project shifted from the design of the interface to include the data collection process, the SWOT analysis revealed that the interface design with its emphasis on data review was a threat to the completion of the data collection and data entry process. While a substantial risk, an additional step was added to the project plan and that was to improve the data collection methods and this change had an impact on every milestone listed above.

\subsubsection{Project Documentation}

Documentation of project was the most valuable and most costly part of the project plan. Project documentation was expected in the following forms:

- Protocol and methods for data collection

- Standards for metadata

- Workflow best practices for data management

- Workflow best practices for GPS project management

- Software specifications

- programming code with variable and object naming conventions

- Paper datasheets

- Field logs and daily summaries of effort

- Photographs

- Manuals for data entry

- Spatial data metadata

- Interface error log

The bigger the project, the quality of the project documentation should be more detailed and better. Goals of creating clear documentation were difficult to compromise, especially when the consequences of insufficient project documentation inherited from the project start created so many unwelcome challenges later

\subsection{Summary}

The design of the system maintained a design goal of integrating a set of spatial data based upon locations measure in the field with a GPS and the diverse and extensive observation data about the biology and physical characteristics of the mapped locations. The storage of the data and the display of the data were combined in a system design that could be maintained in an existing environment of software for ongoing use and maintenance by National Park Service resource managers. A plan was made based upon 
the analysis of expected use and the constraints of existing conditions, the experience of expected users, and limited budget for software license or royalty payments. The design analyzed all five of the major components of a GIS to set expectations of proper function and it anticipated that its use would change based upon the documentation that drives the collection of data and the documentation the system requires to inform the users about its operation. The system analysis and design was extensive and comprehensive and this promoted its implementation. A pilot project was planned using the design for gauging the design's success. Changes in the environment and the responsibilities to manage the project had a large impact upon the design and plan. 


\section{Chapter 4 - Database Design}

The database design is discussed in two parts with the database designed to hold observation data discussed first in section 4.1 and the GIS database second in section 4.2. Design for the two types of databases was done to improve the storage of observation data and the retrieval and connection of the two databases by the database interface. Data that populated the two databases came from different sources. The data that originated from the field observations and data obtained from other sources is detailed in section 4.3 including methods of processing, conversion or documentation that were used to prepare the data for loading into the databases that represent the Coastal Biophysical Inventory.

\subsection{Access Database}

This section describes the design of the database that holds the Coastal Biophysical Inventory tabular data. The database design distilled down to six essential parameters with the reasoning behind them is summarized in the following table, Table 3. 


\begin{tabular}{|l|l|}
\hline Parameter of function & Reason for Parameter \\
\hline It must hold the data type & $\begin{array}{l}\text { There are many types of data, text, integers, numbers with } \\
\text { double precision, binary raster data as images, paper } \\
\text { documents, coordinates, spatial features, etc. While almost } \\
\text { all formats can be held within an Access Database, it excels } \\
\text { at storing text and integers in tables. }\end{array}$ \\
\hline It must be relational. & $\begin{array}{l}\text { Because the essential goal is to observe the coast before } \\
\text { damage occurs in order to compare it to observations after } \\
\text { damage occurs, the data is at its origin a many to one } \\
\text { relationship of observations to a place. }\end{array}$ \\
\hline $\begin{array}{l}\text { It must be able link to the } \\
\text { external GIS data }\end{array}$ & $\begin{array}{l}\text { Based on the protocol, the organizing structure to the } \\
\text { location information of the data is substrate. The } \\
\text { combination of different sizes and shapes of substrate define } \\
\text { it as a place. Each place becomes the pivot for the database, } \\
\text { and the unique number for this place becomes the relational } \\
\text { link between the tabular data and GIS data. }\end{array}$ \\
\hline $\begin{array}{l}\text { It must be able to link to } \\
\text { the external objects data. }\end{array}$ & $\begin{array}{l}\text { Because of size limits and performance limits to the Access } \\
\text { format database, larger data types such as the GIS and image } \\
\text { files need to be stored external to the database, but fixed in a } \\
\text { location relative to the database so that it can retrieve the } \\
\text { data using paths that are relative to the database files own } \\
\text { location. }\end{array}$ \\
\hline $\begin{array}{l}\text { It must reinforce the } \\
\text { integrity of the data as } \\
\text { defined by the protocol. } \\
\text { sheets to the database, there is great opportunity for data } \\
\text { entry error. Validating or restricting the data options greatly } \\
\text { improves the integrity of the data by excluding the } \\
\text { opportunity for data entry error. }\end{array}$ \\
$\begin{array}{l}\text { The database must be able to allow users at two different } \\
\text { locations enter data. Because of its size, online hosting was } \\
\text { not an option and due to unreliable network connectivity and } \\
\text { FTP site changes, the database could not be situated in one } \\
\text { spot with multiple concurrent connections. Data entered into } \\
\text { one must be able to be integrated into the other. }\end{array}$ \\
\hline parame
\end{tabular}

Table 3. Data functional parameters and reasoning for database design. 


\subsubsection{The role of the segment}

The segment, in a sense, holds the observation. However, the segment does not exist until the first observation instantiates the segment by the field biologist classifying the substrate at a location. This act of classifying the extent of a type of substrate bootstraps the segment into existence. From then on, the segment can contain observations of that location and relate to other data. Each observation holds multiple attributes. The result is that the database holds many segments, each segment holds many observations and each observation holds many attributes. It is much like the Russian folk toy of nesting dolls (matryoshka), where the larger doll encapsulates the multiple smaller dolls. In fact it is like whole families of nesting dolls (the observation records) fitting into one big doll (the database). See Figure 5 for a diagram representing the nested data and database concept.

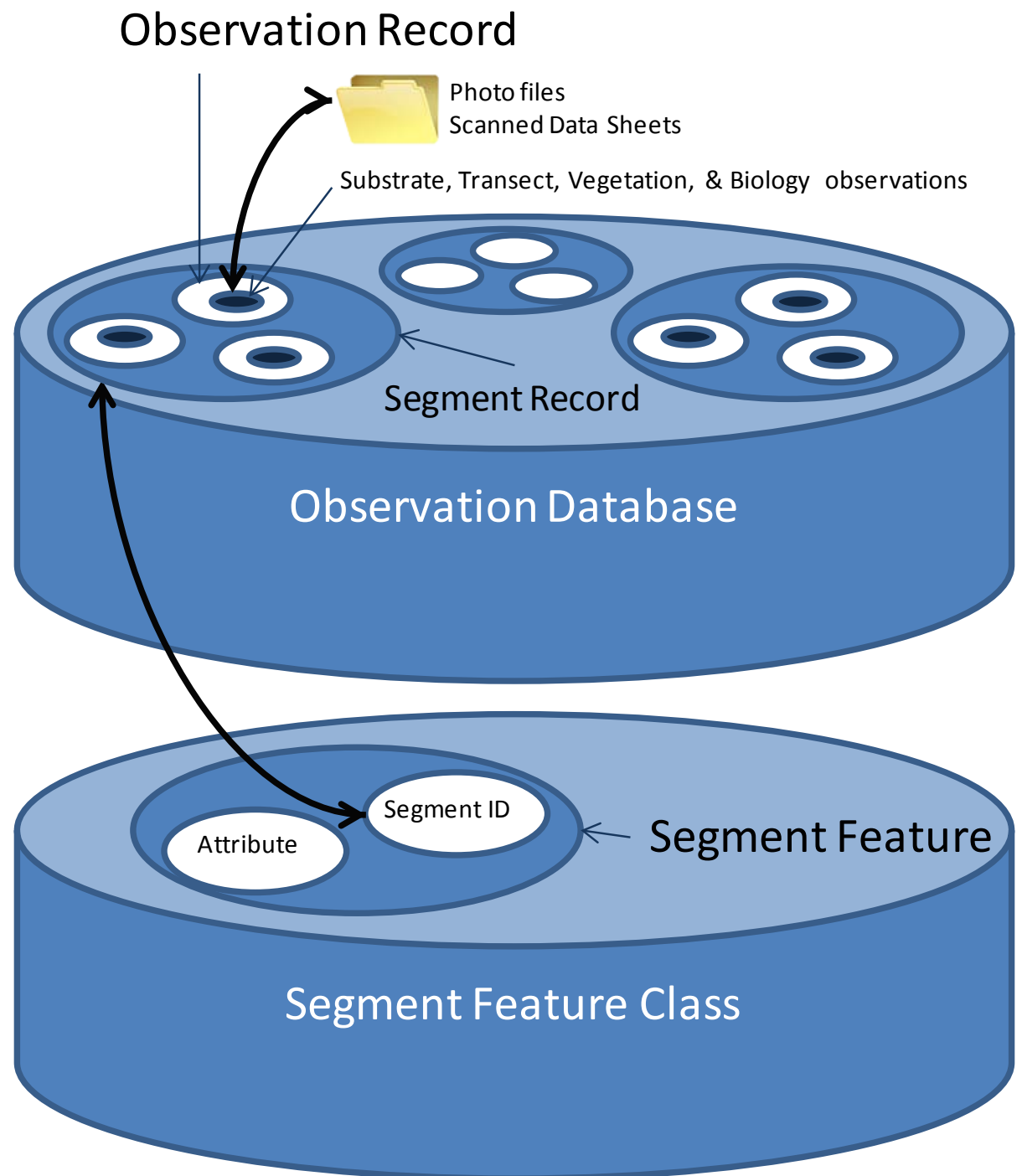

Figure 5. Nested container database analogy 
The nesting is organized by a cascade of linkages. The first and primary table in the database is the Segment Table. It holds an indexed collection of unique identification numbers for each and every segment. This unique number is created in the field when the researcher types a part of the coastline to a particular substrate combination. A data sheet of observations is given the number and GPS coordinates are collected for its upcoast and downcoast limits and given this same number (Upcoast and Downcoast are terms of topology applied to the Coastal Biophysical Inventory. Upcoast is to one's right while standing on land and facing the sea, downcoast is to one's left.) The table holding the segment information will not allow duplicate segment numbers, so that if a field observation is accidently given the same number twice, then it cannot be entered until the GPS coordinate's segment identifier and the datasheet's segment identifier are both changed to a new and previously unused number.

\subsubsection{The Observation Records}

The second and most important table is the Main Segment Observation Table. Each observation is assigned a Global Unique Identifier (GUID) and the Segment ID number to which the observation is about is attached to that GUID in the same observation record. The GUID allows for unique sequential numbers and is the key to linking the related observation data. A GUID is essential for combining replica databases from two different locations back into one. If only a sequential unique number is used for the primary key of the record, then when the databases are combined on a later date, the data fails data validation because the identifiers for the observations are most likely no longer unique (there is an excellent chance both data sets start numbering at 1). In theory, the GUID is based on a unique combination of a number of serial numbers unique to the user's computer's network ID card, processor, username, etc. This means that no two machines will ever enter the same GUID as any other machine, nor will it ever repeat a GUID on the same machine. It maintains data integrity because the data manager can combine data into a database without conflating data, keeping each unique observation record unique. In the off chance that a duplicate GUID is created, the existing database will reject any GUID that has already been added. This is useful for two reasons. It catches the wildly unlikely chance of duplicate GUIDs. Also, if an "append to table" batch process is accidently done twice, it rejects any duplicates. The data that relates to the observation in a one to one way (for example one date for observations do not span more than one day according to the protocol ) are also entered in this table to keep the database compact in size, simpler in its complexity, reduced to its essential elements in structure. This is also known as normalized.

Main Segment Observation Table uses a double primary key. The combination of the unique segment number and the GUID observation number reinforces the ability of the data to make many changes, have multiple updates from replica databases, and reinforces referential integrity of the database during cascading deletes or updates without duplication or mis-relation of data to other tables. Each table that connects to the Observation Table (e.g. Photos, Transect, Biology, Streams, Vegetation, etc.) and is a subset of that observation also has the same double key. The double key is a denormalized trait designed into the database. The database could make use simply of the GUID key linked to the Observation table and related tables, however if one wanted to simply sort the Biology Table by Segment ID they would have to build a more 
complicated query to join the three tables together through the GUID and the Segment ID. GUIDs are not user friendly to read, they are machine friendly to create. The Segment ID tagged on to each GUID helps a viewer quickly browse related tables visually and see the relationship to the Segment immediately. For example if a Segment number is 02DS04 (a code representing the fourth observation made by Dave Schirokauer in 2002), then the GUID for an observation for that segment might read “BD2F6549-113F-4EA9-94E8-5447ED486EB6" which is very difficult to remember or recognize later. The ease of recognizing the Segment ID promotes making visual connections between data that might inspire systematic inquiry into the patterns of linkages observed. The denormalized use of the two primary keys of the GUID and the Segment ID creates redundant data, but that is negligible as a data storage constraint compared to the ease in workflow it creates.

The use of the double key also allows connection of observation tables to external GIS data without lengthy Structured Query Language (SQL) format sentences can be difficult to write or correct for error due to syntax or spelling errors in their construction. This decision to denormalize parts of the database assisted with browsing the many tables related to observations and the linking to outside data sets with simpler SQL query sentence structure. The SQL statements in figure 6 are examples of queries with identical results that display the names of all organisms (BIO_NAME) in the biology observation table that match the Segment ID in the Segment table's field (SEG_ID). Because the denormalized version must link the biology table to the segment table through the main observation table, it is a more complex syntax to edit. Because a simpler query sentence structure was desired for easier SQL statements to edit or to simplify links to the tabular data in external databases, the denormalized table with the Segment ID was developed. Also, the denormalization had the additional benefit of supporting cascading deletions of data if a segment was removed or changed its ID during error correction.

Normalized Database SQL statement:

SELECT CBI_tbl_SEGMENT.SEG_ID, CBI_tbl_SEG_OBS_BIO.BIO_NAME FROM (CBI_tbl_SEGMENT INNER JOIN CBI_tbl_SEG_OBS_Main ON CBI_tbl_SEGMENT.[SEG_ID] = CBI_tbl_SEG_OBS_Main.[OBS_SEGMENT_ID]) INNER JOIN CBI_tbl_SEG_OBS_BIO ON CBI_tbl_SEG_OBS_Main.[OBS_SEGOBS_PK] = CBI_tbl_SEG_OBS_BIO.[BIO_OBS_GUID];

Denomalized Database SQL statement:

SELECT CBI_tbl_SEGMENT.SEG_ID, CBI_tbl_SEG_OBS_BIO.BIO_NAME FROM CBI_tbl_SEGMENT INNER JOIN CBI_tbl_SEG_OBS_BIO ON CBI_tbl_SEGMENT.SEG_ID = CBI_tbl_SEG_OBS_BIO.BIO_SEG_ID;

Figure 6. SQL statement comparison 
Each table that relates to the Main Observation Table has the double key described above, but only the MAIN Observation table makes that specific double key a double Primary Key. The related tables use the GUID and the main observation field for that data as a double Primary Key. For example, the Biology Table has a GUID field to connect to the observation and a Species Name field for the observation. The Observation table's GUID is a unique number linking the Biology Table. Because there can be multiple observations for the same segment, the Segment key allows duplicates in the Biology Table. However, one would never want to enter 4 Ochre Stars, 4 Crabs and then 2 Ochre Stars for the same observation. It should be entered as 6 Ochre Stars and 4 Crabs. If a data entry person isn't paying attention, they might enter the same crab observation twice in a row after stopping to answer the phone and picking up where they left off. By combining the Species Name and the Observation GUID into a dual Primary Key, the database rejects incorrect duplicate entries, because the combination of these two is set to be a unique pair. 


\subsubsection{Data Integrity}

Referential Integrity, cascading deletes and cascading updates can happen in this database because the relationships are built upon these double keys throughout the database. If someone deletes a Segment, then all observations for that segment are deleted. Using the nested doll analogy again, if you throw out the big doll you also discard the many smaller internal dolls. That is a cascading delete. A cascading update occurs if the user updates the Segment ID, then all related observations and the Observations Table's related tables also update. This is unlike the nested doll analogy. If you paint a mustache on a nested doll's biggest doll, all the smaller dolls inside do not get mustachioed too. This is quite helpful for a database manager who needs to correct or update the spelling of an entry. Change the database in one place, and all the places in the database where it should be updated are automatically corrected.

The remaining database design includes three other types of tables. They are legacy data held in its original format for reference, tables used for holding the results of queries temporarily, and domains in the form of look up tables.

Legacy data is important because many choices were made when transforming the data and conforming it to the revised protocol. Some of these revisions were simple, such as splitting the Wave Swell field that read " $2 \mathrm{~m} 312$ deg" into a Swell Meters $=2$ and Swell Direction = 312, others required changing a binary "Yes, No" to a string of choices "Yes, No, Not observed, Not Applicable." Maintaining the legacy data allows a data auditor to observe the originally entered data if desired.

Some controls on the user interface cannot connect to virtual tables held in the computer's memory as a record set or array; they are designed to only connect to other objects in the database. An example of this is the drop down combo box used liberally throughout the database interface. Usually the source for the list is obtained from a table that can be updated. The Data Entry Person's Name is an example of this. When a user updates the Data Entry Person's Name table, the combo box is updated when the control is refreshed. The drop down combo control can also be filled programmatically when it is first created. If a database user creates a record set in memory and fills it with the results of a query (e.g. SELECT all Segments that have Seal Sleeping Areas in their observations and sort the row alphabetically) the combo box cannot fill itself with the results of that query because it does not have access to the reference for that memory location in the Visual Basic for Applications language. The solution is to create a table to hold the results of queries like this temporarily so that the combo box can have instant updates, improving workflow.

To visualize the relationships that are part of enforcing data integrity, see the "Coastal Biophysical Inventory Database Table Relationship Diagram” referenced in Appendix C and also located in the Coastal Biophysical Inventory "Protocol" folder in the CBI data set. In that document, the table representing the attributes of the CBI_Segments feature class is shaded light blue. The main observation table which coordinates the individual observation records as single records is colored light orange. Observation records stored in a many to one relationship to the observation record (e.g. multiple photos for one location) are colored light green. Domain data used to enforce data integrity in the storage and entry of data into those tables are represented in white 
boxes. One to one relationships between tables are represented by a solid line and many to one relationships are annotated with an infinity or one symbol. In figure 7, a snapshot of database relationships is provided to show the overall structure and guidance in interpreting the supporting document. For details and improved legibility of elements please refer to the original document the snapshot represents. 


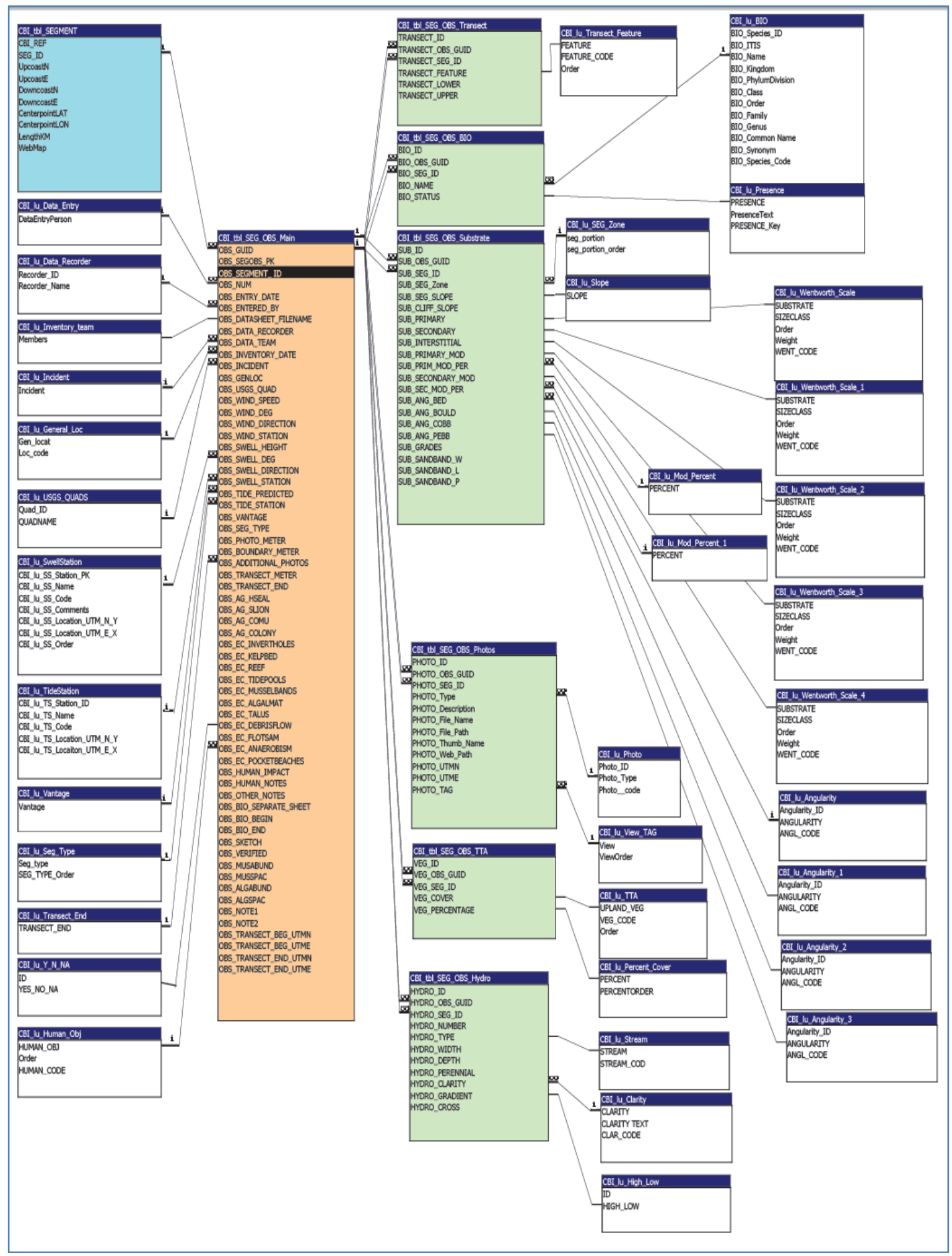

Figure 7. Database relationships diagram snapshot 


\subsubsection{Domains}

Domains are the expert data. Domains help maintain data integrity similar to the referential integrity settings using the Primary Keys described above. All the domain tables in this database are called "Look Up" tables. If a person types their own name 150 times during the course of data entry, there is a pretty good chance of making a mistake typing it incorrectly. If there are hundreds of fields in the database with even lengthier entries such as Anthopleura xanthogrammica the risk of incorrect spelling becomes unacceptable. In many cases throughout the data entry interface, the combo box auto completes and autocorrects the data entry person's typing to match the list of accepted spellings. Limiting the possible options of entry makes data entry easier and maintains a higher level of error free data.

Because human experts will be viewing the data and the fact that there is plenty of expected room in the database for growth, a few aspects of the database were left denormalized. In a few situations, the data is left denormalized in its idiosyncratic format because the complexity of converting the legacy data to the new desired format was more effort than it was worth compared to other project demands. Other changes were not made with the "If it isn't broke don't fix it" approach to project management. This was true for the biology data.

Creating a unique taxonomic identifier was not included in the design of the Biology section of the database, but was considered at length. Taxonomic serial numbers relate to the most precise identification one can give to an organism. They are an abstraction of the organism. If one can identify an organism down to species in the field, a species name is noted and the taxonomic identifier is entered into the database. Occasionally species names are revised; the species is split into two species, lumped into one, or the name reverts to a name given previously in time due to naming conventions.

If a taxonomic ID is used to complete the organisms observe red table, then when the species name is updated on the lookup table, no cascading update is required. In the $\mathrm{CBI}$ database the full text of the organism name is given at the taxonomic resolution possible in the field and this additional use of characters in long scientific names repeatedly "bloats" the database. It also makes it less efficient to search than a taxonomic database table designed with a unique and indexed serial number. For the estimated size of the final database, the performance improvement for this database would be negligible if changed compared with the work to redesign it, so the decision was made to leave the conceptualization of the taxonomic data as it was with the potential to change at a later date.

If changed at a later date, the database design would prioritize the ability to relate it with the growing success of All Taxa Biotic Inventories (ATBI) and taxonomic names databases such as the Integrated Taxonomic Information System (ITIS). (Integrated Taxonomic Information System 2009) Currently these databases do not cover the complexities of the taxonomy of intertidal marine invertebrates and do not list a majority of the organisms expected. The Coastal Biophysical Inventory taxonomic names standards now conform to the Partnership for Interdisciplinary Studies of Coastal Oceans (PISCO) current taxonomic tables provided by the PISCO group at UCSC. 


\subsubsection{Non tabular data}

Finally, due to the total size of the digital photo dataset increasing with each and every segment and the resolution doubling for each camera model used throughout the history of the project, large binary data (aka. BLOBS) were designed out of the database. The other BLOBs excluded were the PDF format files of every paper field data sheet. A minimum of four photos were taken of each segment and two datasheets for each observation. At an estimated 800 segments, this will be a large dataset. The database was designed to relate to a tree of folders adjacent to and below the database. This was explicitly done so that relative paths to the objects could be stored in the database as file paths in a text format. Programmatically the database can see where it is in the file structure of the computer and then access these related files through their relative paths. This means that JPEG or PDF format files remain stored in their compressed state rather than being enlarged into their uncompressed state in an Access database as a BLOB, making the final entire dataset much smaller.

\subsection{GIS Database}

The fundamental and single most important element of the GIS database is the spatial representation of the coastline inventoried as a line feature and is described in the conceptual data model section. The second essential element of the GIS database is the storage and organization of information to overlay the coastline line feature over for orientation, analysis and visual interpretation of neighboring features. This second element includes the details of the segment feature structure and the folder structure to store. The base data set for rapid comparison is also stored in a Personal Geodatabase for assisting the use in ArcGIS projects. Both the folder structure and the Personal Geodatabase are described in the Logical Data Model section. The third element of the

GIS database is the organization of the project workspace to host interim data sets and the messiness of multistage data organization and experimental scratch space. The third element is not described as it was not part of the deliverable data set, but the folder “CBI_ArcGIS_Projects” (see figure 8) was included as a place to organize projects in subfolders when authoring maps or analysis using the CBI dataset. The projects are not included on the DVD accompanying this document.

\subsubsection{Conceptual Data Model}

The feature that represents a section of the coast is called the CBI Segment. It approximates the CBI's inventory and mapping protocol's sectioning of the shore using the criteria for assessing breaks in substrate type to estimate a transition in substrate type. Each feature should be assigned a unique identifying number in addition to its unique feature number that allows relation to the tabular database holding observations about the location it represents as a foreign key. It needs to store additional attribute information unique to that location and varies little over time.

Data other than the CBI Segment will inherit the source data's structure and will be stored as both separate feature class files and redundantly in a Personal Geodatabase. 


\subsubsection{Logical Data Model}

The GIS database logical design fell into two priorities:

1. the feature class for the process of storing and ongoing editing of the CBI Segment polyline

2. the storage of existing raster and vector data layers anticipated in the analysis of the coastline in comparison to the CBI Segment.

\section{CBI Segment Feature class}

After the unique CBI Segment ID is assigned to the feature that represents the coastline of a substrate type, it is complete for the GIS. However, additional attributes will be needed for display in the interface and these additional attribute data should be stored with the CBI Segment feature class so it is distinct from the many observations that are about that place. Attribute information about the CBI Segment is placed in the feature class so that it does not repeat it in the tabular database of observations needlessly. Because this changed the conceptual model, an extension of the feature class to include these attributes and their purposes in the CBI Segment feature class is summarized in the following tables 4 and 5 :

Table 4. Feature class properties for CBI Segment

\begin{tabular}{|l|l|l|}
\hline Name & Property & Reasoning \\
\hline $\begin{array}{l}\text { Name: } \\
\text { Alias: }\end{array}$ & $\begin{array}{l}\text { CBI_Segments } \\
\text { CBI_Segments }\end{array}$ & $\begin{array}{l}\text { Simple, thematically } \\
\text { identified }\end{array}$ \\
\hline Type & Line Features & $\begin{array}{l}\text { Represents coastline. If a } \\
\text { polygonal representation of } \\
\text { the coastline is desired, the } \\
\text { coastline can be buffered } \\
\text { along the length of the } \\
\text { transect to the terrestrial } \\
\text { environment to approximate } \\
\text { the shore area. }\end{array}$ \\
\hline $\begin{array}{l}\text { M Values? } \\
\text { Z Values? }\end{array}$ & $\begin{array}{l}\text { No } \\
\text { No }\end{array}$ & $\begin{array}{l}\text { approximately 0 mean sea } \\
\text { level. }\end{array}$ \\
\hline $\begin{array}{l}\text { Projection: } \\
\text { Geographic } \\
\text { Coordinate } \\
\text { System: } \\
\begin{array}{l}\text { Datum: } \\
\text { Spheroid: }\end{array}\end{array}$ & $\begin{array}{l}\text { Transverse Mercator (UTM) } \\
\text { GCS North American 1983 }\end{array}$ & $\begin{array}{l}\text { NTM zone 10 north, NAD 83 } \\
\text { is the preferred format for the } \\
\text { projection and coordinate } \\
\text { system by PORE and GOGA. }\end{array}$ \\
\hline
\end{tabular}


Table 5. CBI Segment feature class attributes and data types

\begin{tabular}{|c|c|c|}
\hline Field Name & Data Type & Reasoning \\
\hline FID & Object ID & $\begin{array}{l}\text { Inherited part of the } \\
\text { geodatabase feature class. }\end{array}$ \\
\hline Shape & Geometry & $\begin{array}{l}\text { Inherited part of the } \\
\text { geodatabase feature class. }\end{array}$ \\
\hline OBJECTID & Long Integer & $\begin{array}{l}\text { A unique number representing } \\
\text { the row number and useful in } \\
\text { creating " relates and joins" to } \\
\text { tabular data in ArcGIS }\end{array}$ \\
\hline Seg_id & text & $\begin{array}{l}\text { The unique identifier that } \\
\text { relates observation to location. } \\
\text { Indexed for performance } \\
\text { improvements. }\end{array}$ \\
\hline Upcoast E & Double precision number & $\begin{array}{l}\text { The UTM easting coordinate } \\
\text { for endpoint of the line to } \\
\text { facilitate additional } \\
\text { observations adjacent to the } \\
\text { segment. }\end{array}$ \\
\hline Upcoast $\mathrm{N}$ & Double precision number & $\begin{array}{l}\text { The UTM northing coordinate } \\
\text { for endpoint of the line to } \\
\text { facilitate additional } \\
\text { observations adjacent to the } \\
\text { segment. }\end{array}$ \\
\hline Downcoast E & Double precision number & $\begin{array}{l}\text { The UTM easting coordinate } \\
\text { for endpoint of the line to } \\
\text { facilitate additional } \\
\text { observations adjacent to the } \\
\text { segment. }\end{array}$ \\
\hline Downcoast $\mathrm{N}$ & Double precision number & $\begin{array}{l}\text { The UTM northing coordinate } \\
\text { for endpoint of the line to } \\
\text { facilitate additional } \\
\text { observations adjacent to the } \\
\text { segment. }\end{array}$ \\
\hline
\end{tabular}




\begin{tabular}{|l|l|l|}
\hline Field Name & Data Type & Reasoning \\
\hline CPT_LON_X & Double precision number & $\begin{array}{l}\text { The longitude coordinate for } \\
\text { the center point of the line } \\
\text { feature in Web Mercator } \\
\text { projection and stored in } \\
\text { decimal degrees format for } \\
\text { submitting to online mapping } \\
\text { programs to view data in } \\
\text { external data sets of the area } \\
\text { near the CBI Segment. }\end{array}$ \\
& & $\begin{array}{l}\text { The longitude coordinate for } \\
\text { the center point of the line } \\
\text { feature in Web Mercator } \\
\text { projection and stored in } \\
\text { decimal degrees format for } \\
\text { submitting to online mapping } \\
\text { programs to view data in }\end{array}$ \\
& Double precision number & $\begin{array}{l}\text { external data sets of the area } \\
\text { near the CBI Segment. }\end{array}$ \\
& & $\begin{array}{l}\text { Approximate length of the } \\
\text { segment in kilometers at } \\
\text { 1:24000 scale. Calculated in }\end{array}$ \\
& & UTM Zone 10 North, NAD 83 \\
\hline Shape_Leng & Double precision number & $\begin{array}{l}\text { Inherited from the } \\
\text { Geodatabase feature class, } \\
\text { meters units. }\end{array}$ \\
\hline & &
\end{tabular}

\section{GIS data, folder structure}

The storage of GIS data in a clearly organized and static structure was needed for the simplification of programming connections to the attribute data. The design of the folders and their adjacency or placement under parent folders was conceived as a hierarchy to serve the project, the programming needs and the ease of navigation for use in other GIS projects. The MapObjects LT 2.0 ActiveX COM control and libraries cannot access Personal Geodatabase files, so storage of the spatial information as separate feature classes in shapefile format is required. A critical part of the organization is that it remains static so that programmatically created paths to the GIS data relative to the location of the database interface software were not broken.

There were not programmatic limits to the names and structure; however, file names and folders with spaces or characters more than 25 characters were avoided for compatibility with the limited view of some file browsers and compatibility with some tools in ArcGIS. Therefore, the organization of the data and the folder structure that contained the data was thematically driven and encouraged the use of meaningful names for browsing the data using file exploration tools of the operating system.

The other data sets included in the GIS database were evaluated for similarity in scale, the ability to provide a unique reference when compared to the CBI Segment and 
the completeness of metadata. All data is expected to have a source scale of 1 to 24000 or larger. NOAAA navigation charts, USGS topographical maps and aerial imagery were included to support trip planning for uninventoried shoreline and orientation to a location before or after an inventory. Data such as boundaries, elevation information, indexes, and hillshade images were included into the GIS database as points of reference to show overlapping jurisdiction, cartographic representation, inset map display and potential analysis. All metadata for the supporting reference data were completed to the standards adopted by the project and sit as XML document with each file or embedded in a Personal Geodatabase.

This storage of reference data with the tabular dataset is done so that an individual wanting to set up a new project rapidly would have a set of files that represent the coast for the extent of CBI immediately on hand. Aerial imagery was selected from larger data sets by location and was selected if any part the footprint of the imagery was within a kilometer of the coast. This allowed trimming the overall dataset size to as small as possible without eliminating the reference information needed. NOAA's National Ocean Service's Office of Response and Restoration distributes data useful for oil spill response in Environmental Sensitivity Index (ESI) atlases that are stored in Personal Geodatabase format and were included into the database unmodified as a reference source. (NOAA National Ocean Service Office of Response and Restoration 2009) It is not seen as a complete set because there are not standards for its completeness, but all data added is complete in its coverage of the coastline inventoried for the CBI.

Folder structure of the GIS database was primarily designed to maintain a standard for programming and data loading or maintenance tasks. The root folder for the Coastal Biophysical Inventory is "Coastal_Biophysical_Inventory" and under it are two folders, one to hold the installation files called "CBI_SETUP" and one to hold the database called "CBI_Dataset." Inside the "CBI_Dataset folder are all the tabular, spatial, imagery and supporting documents for the database and interface. This logic and idiosyncrasies of the folder structure is most easy to perceive when illustrated graphically, please refer to figure 8 for the folder hierarchy used for the database as a whole and refer to the folder "CBI_GIS" and its subfolders for the folder structure for the GIS 


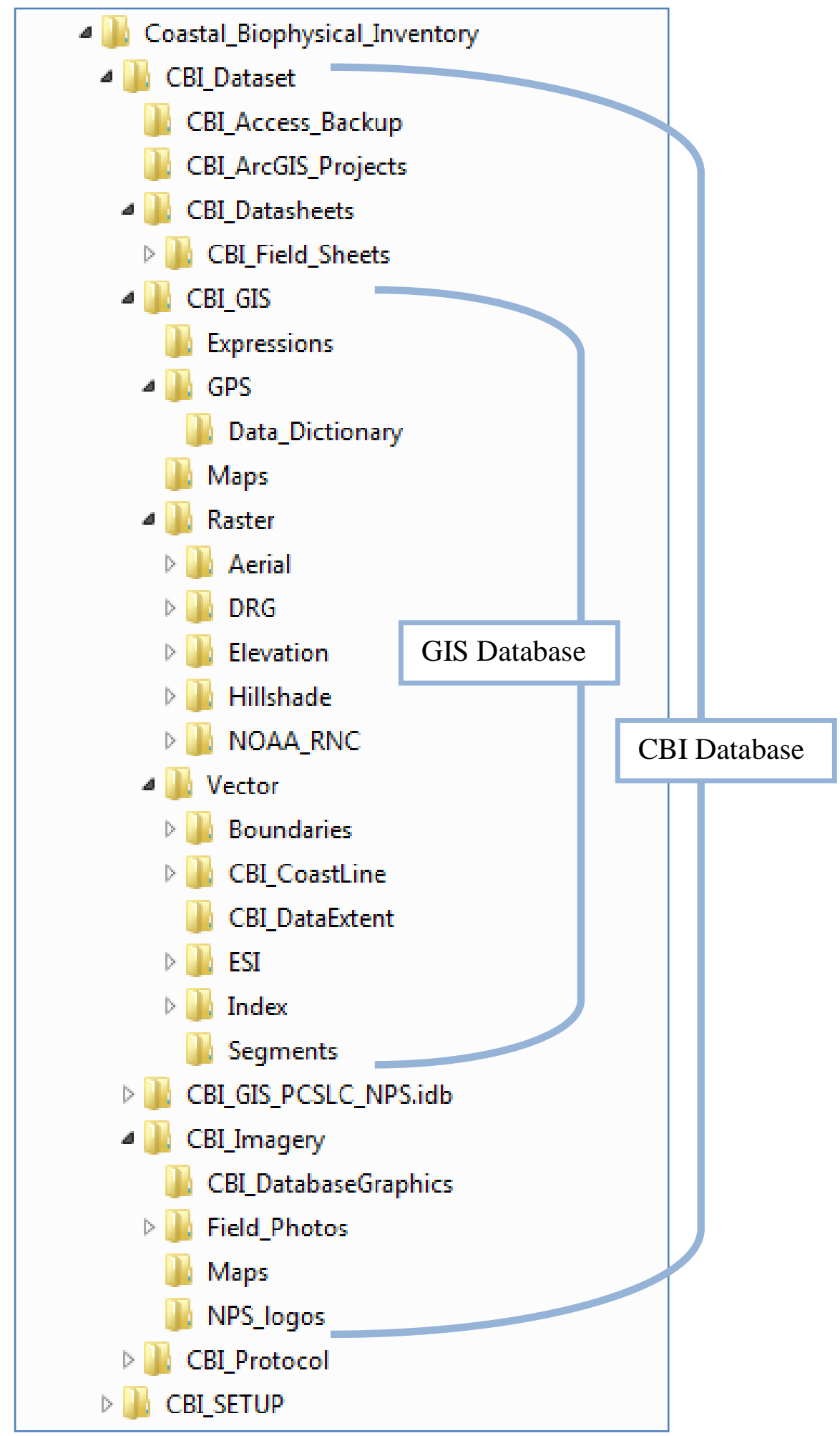

Figure 8. Database Folder Structure 


\section{GIS data, Personal Geodatabase}

The GIS Personal Geodatabase was much simpler to design, as most of its design was inherited from the Personal Geodatabase class. The majority of analysis and ArcGIS connections were made using ArcGIS 9.2 Personal Geodatabase in the .mdb format as the geodatabase format. The Personal Geodatabase was labeled "CBI_GIS_PCSLC_NPS.mdb” and sits adjacent to the tabular database labeled “CBI_OBSERVATIONS_PCSLC_NPS.mdb” inside the "CBI_Dataset” folder. The “CBI_Segments” data and all the supporting reference files and metadata were loaded into the Personal Geodatabase. If edits are made to the data in the Personal Geodatabase, the edited feature class is exported as a new version to the GIS database and can be stored in addition to the previous version or replace it and update the metadata.

When editing and exporting the "CBI_Segments" feature class to a shapefile in the GIS folders, the previous version of the shapefile is renamed and the "CBI_Segments" file is replaced. The expectation is that this use will be a rare task because edits beyond correcting the representation of location (e.g. add, delete) or attributes of existing segments (e.g. change Segment ID) must be followed by edits to the observations database or new data entry in order for the two data versions to remain synchronized.

Because the high resolution imagery is much greater in size than an ArcGIS Personal Geodatabase can hold, the raster imagery was linked as raster catalogs in their unmanaged format instead of imported into the Personal Geodatabase. For this reason, deleting the raster catalog in the Personal Geodatabase does not delete the raster object occupying the storage media. The creation of an unmanaged raster catalog makes the addition and symbolization of the data much simpler and allows superior management of the reference layers for comparison than if the aerial imagery was loaded one image layer at a time.

Relative linking is expected for this database and portability is also expected. For these reasons, the GIS data were organized like the observation photo data and it sits in folders adjacent to or below the Personal Geodatabase. This is so the user can quickly repair the Personal Geodatabase's references to linked data stored in the neighboring folders in the event it is moved to another storage media and is accessed from an ArcGIS Map Document. While this solution doesn't eliminate all problems with moving a geodatabase with unmanaged raster catalog connections, it greatly enhances the portability of the whole dataset.

The Personal Geodatabase is designed to be separate from and redundant to the spatial files used by the MapObjects components in the database interface. The workflow to keep the Personal Geodatabase in synchronization is limited to the "CBI_Segments" feature class only. The GIS database and the Personal Geodatabase design is to support the ongoing editing of the Segment ID file and to allow the replacement of functions that were part of the functional requirements of the customized database interface by shifting them to ArcGIS

The logical model described was successful in the creation of the database and the use of the data stored in this way will be described further in the implementation section and supporting documents. The goal to store and edit the CBI Segment spatial data so it can be accessed via both a MapObjects LT 2.0 based map interface and ArcGIS was 
accomplished. Additional spatial data were stored in a consistent way to facilitate unanticipated use. Folder structure supported managing reference data relevant to the extent of the Coastal Biophysical Inventory so that analysis and review were not hindered by new users unfamiliar with finding and organizing available data. They hybrid use of MapObjects continues to allow access to CBI Segment spatial data for browsing and simple queries without an ArcGIS license, but this design is partially a solution to the original requirements and would likely be deprecated and replaced by a connection between Microsoft Access and ESRI ArcGIS if redesigned. 


\section{Chapter 5 - Database Interface Design}

In Microsoft Access, the user interface derives its form from the functional limits of the user controls, the programmatic interaction between user forms and subforms, the underlying database structure to which the user controls are bound, and flow of reading and entering data from a data sheet. Space constraints on a page and the complexity of the data made for a challenging user interface design. When leadership of the project changed, the emphasis shifted from displaying existing data to getting researchers into the field. When staff returned from the with the observation data, the corollary to the change in emphasis to support ongoing data collection was to get the new data stored in a database. The user interface design was crucial to meeting this goal.

\subsection{Interface design inside a parent application}

Microsoft Access software comes with many tools and toolbars that are as useful as they are potentially destructive in the hands of new users. Hiding these tool sets from the average database entry technician protects the data and restricts the already complex task of data entry to a less cluttered interface experience. This step of hiding unneeded tool bars also liberates large amounts of the visual "real-estate" of the monitor and allows for less crowding of elements. Less crowding of elements allows the use of negative space to imply sections, and gives room for larger buttons and fonts for tired eyes. This improvement was removed when the interface for Microsoft Access changed dramatically from the drop down menu structure to the ribbon toolbar design released in Microsoft Access 2007 and continued in Microsoft Access 2010. The CBI Database interface design coordinated with the ribbon toolbar design better and the toolbar management through software code was removed.

\subsection{Guidelines to CBI database interface design}

The observation record is an extremely complex set of data. The data entry experience is a critical part of maintaining the integrity of the data when transcribing it from the paper data sheets to the computer. The interface design for the Observation records followed some simple guidelines:

1. Using the TAB key, advance through the controls where data entry should occur in the same top to bottom, front to back, and page to page reading order of the paper field data sheet.

2. Dust off the tired Windows 3.1 look of the common and often garish Access Database user form with pleasing fonts and subdued colors.

3. Use tabbed page controls to logically divide the sections of the interfaces to match sections of the field datasheets and facilitate an easy to read flow of the interface that is familiar to the user.

4. Use continuous, repeating forms to present data that is linked through a many to one relationship.

5. Provide quick access to regularly updated domain/look-up tables.

6. Validate data on entry to reduce error and provide tool tips.

7. Auto-complete and provide easy to access choices via keyboard or mouse click. 
8. Allow for empty data, but signify when data is empty by conditionally formatting the control's color.

9. Visually nest the controls so that they parallel the nested structure of the database.

10. Allow access to the visually rich data set that accompanies each observation.

11. If data is limited to a list, always provide that list for selection.

12. Set default data for as many options as possible.

13. Every keystroke reduced during data entry is a victory.

14. $1280 \times 1024$ is the highest resolution setting common to most LCD screens available through the Government Services Account purchasing account and used by the National Park Service. The interface should be designed to this screen resolution.

\subsection{Representing the multiple observations of a single record}

As illustrated earlier in the database design section, the database has a nested conceptual structure. The interface design mirrors that structure. The main data entry form (navigate to this using the second choice in the navigation bar on the left of the interface display) displays a single Segment and its related attributes. Advancing through the Segment records is easily accomplished using the default record navigation buttons provided in Access on the bottom left. Using the nested dolls analogy again, imagine placing a set of nested dolls on a kitchen Lazy-Susan. Advancing through the database record set to the left or right is analogous to spinning the Lazy-Susan to your desired set of nested dolls.

When the user advances to a segment, the observations for that segment need to be immediately viewed and edited. The main form houses a subform window. The data records between the two are connected so that advancing to one record immediately displays a form that holds all the observation records as a subform. If there are no observations for that record, the window is empty. This interface design idea was done in order to visually reinforce that the observation record is not there when the window is empty, rather than have a page of empty controls. For this interface, browsing capability and data entry go hand in hand. Not all data will or can be entered at the same time, requiring the user to be able to come back and see where they stopped or may need review for corrections by database managers.

Subforms are an essential design solution in the Access database user interface design environment. There are an approximate maximum number of controls that a designer can add to a form, ever. This includes controls that were deleted. It counts the times you add a control to a form, not the current number residing on the form. On a complex user interface such as this, the complex controls were always intended to be split to subforms, which only count as one control on another form. This way the maximum number of controls was avoided and the database opens, closed and compiles without error. The subform mechanically serves as a nested relationship via the records in the subform relating to the parent form. It simplifies the display of many records to one record in a repeating way that allows thematic grouping of data visually. It allows interface design for complex data sets that are otherwise impossible with object control limits in simple single forms. 


\subsection{Interface design and user feedback}

The design the interface designer intends for the user the best experience and function. That experience may be greatly improved by incorporating the comments and requests made by the user. Expectations of the data user and real use examples greatly influence the rapid improvement of the interface design but "Bug reports" are part of the feedback that creates a better interface. If the interface designer responds quickly and thoughtfully to the comments or requests of the user, they are inspired to be more thoughtful when articulating their comments because the user feels are being heard and become invested in the success. Fixing their requests may not be immediate, but the response to their request should be. User comments should be viewed as golden opportunities rather than complaints. The user database interface and its correct functioning have been greatly improved by feedback from the users.

\subsection{Organization of Database interface}

Because the database will possibly expand beyond data entry in future versions a database navigation switchboard was created for the left side of the interface display. A table of contents like structure was used because it is common to many user interfaces and is an easy concept for new users of the database to grasp. The navigation switchboard allows one to quickly see other parts of the database and display them. This is an improvement over the common switchboard structures found in many Access databases that require the user to close the window they are viewing in order to see the switchboard and then switch to the next section of the database they want to see and hide the switchboard. This design streamlines a common database navigation task.

From the first interface of the Hummingbird pilot database to the VB6 application (discussed later in the implementation section) to the present day interface for the Access format Coastal Biophysical Inventory Database, the interfaces had a common theme of the tab. The tab control can easily be overdone, (Hayes 2000) but if used sparingly tab controls can divide large amounts of data into the small window of the average monitor. Data entry can be facilitated by creating an interface that models the underlying data structure in the structure of its layout, but this goal is limited by the functional limits of the controls to display the data. Not all success in interface design comes from layout. If an interface looks good, but advances awkwardly through the data it, performance generally needs to be prioritized over aesthetics. The greatest improvements to performance and aesthetics can come from listening earnestly to the comments of the users of the interface. See figure 22 for a diagram of how the design emphasis changed over time from data review to data entry as the different programming environments and software were used to respond to the protocol that directed the data collection

\subsection{Summary}

The design of the Coastal Biophysical Inventory as a system became broad in scope compared to its original task of introducing a map interface into the database interface. While beyond the scope of this document to define its creation, the "Coastal Biophysical Inventory (CBI) and Mapping Protocol" was essential to the design of the system for the tabular data in the observation database, the GIS database, and the interface design. 
Standards were developed and data or metadata were adapted to conform to those standards for loading into the database and use during the collection of data. While a project is in progress design goals and data are abandoned that may have accounted for a significant portion of the work or were interstitial to the creation of the final data and are part of the documentation of design and process. Considerable efforts were made to collect high quality observations as rapidly as possible and the system design always put the maintenance or improvement of data quality during the entry and electronic storage at the highest priority. Database interface design was to reinforce that priority while improving user interaction, but found constraints on aspirations to improve interface design. 


\section{Chapter 6 - Data}

As a system designed around the storage and display of data, the data intended for use in the system influenced design. Data needed to be acquired, and processed before loading into the database. The acquisition of data and conforming it to the standards and structure of the database was a significant and ongoing effort during the Coastal Biophysical Inventory. This section chapter will summarize the data sources, collection \& compilation of data and the preparation of data for inclusion into the database and display in the interface.

\subsection{Data sources}

There were many sources of data. Some data were collected by the participants in the Coastal Biophysical inventory that were both spatial and tabular records of the observations in the field. Much of the spatial data organized from existing data that was trimmed to the area of interest from larger data sets. Some data needed conversion or transformation to be used in concert with segment location data. Data of similar scale and quality was obtained to provide complete coverage of the full extent of the inventory location.

\subsubsection{Collection and Acquisition}

Data collection was a primary task of the Coastal Biophysical Inventory Project, and the database to hold and allow entry of the data was integral to the system design. Because of prior experience as an environmental educator of intertidal ecology, there was sufficient knowledge of biology and field collection methodology for me to participate in data collection and identification of substrate and organisms. The hands on experience allowed a pragmatic understanding of its source. The experience also motivated the improvement of workflow by seeing when and where the system could be corrected.

Data collection was managed as a collaborative effort with PISCO staff trained in identification of all marine organisms of the intertidal zone along the California coast that are visible to the naked eye. My participation in data collection occurred to verify or clarify the protocol methods, training in use of mapping equipment (e.g. GPS, transect tape, inclinometer, field log), and troubleshooting integrated design issues by comparing use of paper data sheets in the field to data entry process. A significant portion of the work of Coastal Biophysical Inventory was the articulation of data collection methods in the "Coastal Biophysical Inventory (CBI) and Mapping Protocol," the "Coastal Biophysical Inventory Field Procedure," and the "Coastal Biophysical Inventory Field Protocol Summary Sheet.” These documents are accessed through the database interface along with the master templates for the data sheets used in the field.

Observation data were obtained by individuals visiting the coastline and following established protocol, and included paper data sheets, GPS measurements and digital photos. Data collected by the Coastal Biophysical Inventory covered approximately 161 kilometers of coastline and represents observations made at low tide (below 0 elevation at mean sea level) from the waterline to the terrestrial area inland from the beach. The 
coastline inventoried is represented in figure 2. It is beyond the scope of this document to detail the protocol methods. Please refer to the protocol document for the breadth and depth of mapping and observation methods that were an essential guide to all aspects of the project.

Taxonomic data used in the protocol and as a domain in the database is based upon the taxonomic uses and conventions of naming updated and edited by experts at PISCO. A list of taxonomy that aligned with the precision of rapid assessment process was provided by PISCO and data from previous data collection was updated or corrected to reflect correct spelling, reasonable taxonomic precision in the field with the naked eye, and taxonomic changes that occurred after the start of the project.

Aerial imagery came from two sources (not including system links to imagery of the coastline managed by external systems such as Google Earth and the California Coastal Records Project). At the end of 2004 the aerial imagery representing the coastline of the Marin Peninsula (from the Golden Gate north) was obtained through the Point Reyes National Seashore's (the agency of the project client) participation in a collaborative effort with a consortium of other agencies located in Marin County. Cost sharing and in-kind donations allowed access to orthorectified imagery with approximately 1 foot pixel resolution. That imagery has since been made available to the public for free via MarinMap (MarinMap 2007-2009). The imagery for the San Francisco was collected under a grant from Homeland Security at approximately 1 foot pixel resolution in 2004 and obtained without fees through the California Spatial Information Library hosted at University of California at Davis (California Resources Agency Geospatial Information Office 2009).

Aerial imagery and map data managed external to the CBI Database but available through links on the database interface are documented at the data sets' host site. Google Maps are managed in a process that makes it difficult to impossible to determine the specific source or sources for the imagery in view, except for the data provider's copyright notice. The California Coastal Records Project oblique aerial imagery is available through connection to the internet with quick links to locations near the center of the CBI Segment being viewed available on the CBI Database interface.

The California Coastal Records Project imagery was originally stored inside the CBI dataset, however was extracted due to management challenges and the advantages of a well managed database being available online. Reliance upon outside systems that do not have obligations for interoperability or compatibility with the Costal Biophysical inventory creates a risk of being unable to access imagery if internet connections or the website is removed. The imagery for the coastline from 2002 is archived separate from the Coastal Biophysical Inventory per license agreement to respond to that situation, but the ongoing management, distribution and lifecycle of the imagery are left to the authors Kenneth and Gabrielle Adelman (Adelman and Adelman 2002-2009).

Information representing borders or boundaries (or other reference imagery) was obtained from the authoring agency (listed in the metadata). Multiple image tiles were selected from larger data sets and were chosen if they intersected the CBI Segment locations buffered to 1 kilometer. Other data were clipped or reduced in extent to include only the features or imagery within the CBI Data Extent (the CBI Data Extent is the footprint of DRG raster graphics that cover the areal extent of the bounding rectangle created by the extent of the Coastal Biophysical Inventory coastline). 


\subsection{Data Scrubbing and Loading}

No data were loaded without being modified to a subset of the Coastal Biophysical Inventory extent, enhanced with metadata documents, or transformed to the base data and projection of the project. A series of data management tasks that included quality assessment and quality control occurred throughout the project. There were equal amounts of data purged as loaded during the management of the project as standards were established. Some data sets required creating new workflow and formats to correct or improve them.

\subsubsection{Metadata management issues}

All the data originally provided by the client for the project were lacking metadata files and this inspired the creation of metadata standards for the spatial data in the CBI Database while under the new management of the Pacific Coast Science and Learning Center. Once those standards were created, eventually all of that legacy spatial data were abandoned and deleted from the project and later replaced with data where the provenance was known and the condition documented. Standards for metadata that guided data management were:

1. all data should have metadata

2. all metadata be FGDC compliant and data created by the NPS should include NPS metadata elements

3. metadata created by the project should include documentation of processing

4. metadata shall be stored in an XML format file with the data

The standards incorporated the completion of the NPS Profile extension to the FGDC standards. NPS Profile extension includes elements that identify parks and purpose in the parks for the data as well as boilerplate text regarding use and liability. NPS metadata standards use are described as, "Metadata describing NPS datasets is based on the Content Standard for Digital Geospatial Metadata (CSDGM) published by the Federal Geographic Data Committee (FGDC). The NPS's NRGIS Program has developed the NPS Profile extension to the CSDGM specifically for documenting NPS datasets. The NPS Profile is the metadata standard that should be used for documenting NPS datasets as it provides a common data structure for NRGIS Metadata Applications and for sharing metadata and datasets.” (Natural Resource GIS, National Park Service 2006)

The Environmental Sensitivity Indes (ESI) Atlases data were included unchanged into the reference data set to avoid management of versions, redundancy of data management protocols in place by the author, metadata responsibility, and ownership obligations. The ESI is a data set that is updated regularly and should be replaced as a whole with a complete and new release from the original author (the National Oceanic and Atmospheric Administration).

There were exceptions to the applications of these standards for data that were not spatial data. Metadata about the photographs are embedded in the Exchangeable Image File (EXIF) format embedded in the header of the image file automatically by the camera and the attributes in the observation record in the CBI Database. Datasheets and documents considered as a type of metadata (scans of paper datasheets, protocol documents, and 
installation files) were not given additional metadata per file. Most data that was purged from the CBI Database was due to the lack of information needed to complete the elements about its purpose and origin.

\subsubsection{Preparing spatial data fundamental to the project}

For the CBI Segment, a GPS measurement records the location of the transition of substrate type where it intersects the tide line as one travels along the coast during the time of observation. The GPS measured location is then used to break a line feature in a spatial file that represents the coastline at as large a scale as available. The line data used in the final database as the data source for snipping was different from the original CBI segments already completed in 2003. For the new GIS database, the coastline was derived from USGS digital elevation model (DEM) data with 10 meter cells and was a uniform and repeatable process documented in the metadata of the CBI Segment feature class. The snipping method is documented in the workflow document "Methods for processing Coastal Biophysical (CBI) field coordinates into line features of the coast" (J. Kinyon 2008).

A new coastline data source was created because inspection revealed that the original CBI segment feature provided by the client lacked written metadata and conflicted with the verbal assurance that it derived entirely from the National Wetlands Inventory dataset. From comparisons with other data sets in the existing project it was obvious that the source of coastline data varied during the editing process previously and likely derived from data sets such as a National Park Service boundary line (of undocumented origin), an early version of the National Wetlands Inventory coastline (U.S. Fish \& Wildlife Service 2009), and USGS digital line graph (DLG) data.

The previous (and undesired) heterogeneity of coast line data sources was normalized to one line feature source at 1 to 24,000 scale by replacing and snipping all previous segments from a new single dataset. The USGS DEM files used to generate the coastline line feature used for snipping into individual CBI Segments had problems inherent in their creation and had errors in their metadata that required correction of the data (e.g. elevation datasets labeled as having units in feet actually being meters) and those existing problems made the process for deriving a coastline less than desirable because the process does not go to the many original sources of the DEM elevation instead. Digitizing a shoreline from aerial photography of a larger scale was considered, but dismissed due to lack of resources to complete. Elevation data of better quality and larger scale was not available.

The lack of elevation data of consistent quality for the extent of the coastline at a scale larger than 1 to 24000 was identified as a data need by this project and by several other agencies which resulted in prioritization of the collection of LiDAR elevation information for the coastline between 2009 to 2010 by the U.S. Army Corp of Engineer in collaboration with the USGS. A refined coastline may be created from this new elevation information and replace the existing coastline feature created for the CBI. Until then, the coastline will be represented at the 1 to 24,000 scale using the existing shoreline derived from 10 meter USGS DEM data provided by the Bay Area Regional Database. 


\subsubsection{Reference Imagery}

The California Coastal Records Project (CCRP) provides imagery of the coastline that overlaps the extent of the Coastal Biophysical Inventory and is organized and presented online. The effort to photograph the same location of coastline is repeated approximately every 2 years to assist with change detection, but has also hosts imagery collected of the same locations in previous years by prior projects. Using the center point attributes stored in the "CBI_Segments" feature, the CBI Database user interface automatically generates a website address in universal resource locator format (URL) and submits it to the default web browser available on the system which then accesses the html document through a query to the server at www.californiacoastline.org over the internet. The image taken most recently and closest to the coordinate submitted is displayed with access to images of the same location from 10 different years spanning 1972 to 2009. The California Coastal Records Project includes California's entire coastline (not including some interior bays or restricted military sites).

Imagery from the CCRP project was stored within the first CBI dataset by design, however size of dataset and ongoing data collection by the CCRP made this a data management task that would not likely be maintained in the future and exceeded functional requirements for size and portability. The connections described above were made to access the imagery through an automated spatial query in the default browser of the computer system. For the California Coastal Records Project imagery, data were loaded and then unloaded from the CBI Database when access to imagery was simplified and management of imagery appeared to be an ineffective and redundant practice to continue.

Marin County orthorectified aerial imagery was maintained in the form obtained from its source at MarinMap with metadata created for each file to complete project standards. The source imagery was provided in California State Plane zone 3 feet Federal Information Processing Standards (FIPS) geographic coordinate system (a type of Lambert Conformal conic projection) with the 1983 North American Datum as its reference and stored in a compressed Multi-Resolution Seamless Image Database (MrSID) format. On the fly projection of imagery by ArcGIS meant that the data seamed together without processing artifacts for overlay and comparison with the CBI_Segments feature class.

Originally the imagery was to be displayed using the MapObjects tools and was transformed from the original projection in its TIFF format using ERDAS Imagine's projection tools and batch processing commands into UTM Zone 10 north coordinate system. By re-projecting each file, the imagery could be viewed in the MapObjects based map interface, but the new projection meant that a collar of black surrounded it because it was projected as a single imagery file and the null data were colored black. Transparency symbology or programmatic alteration of raster symbology settings to assign "no color" to the null data represented in the raster image were not properties exposed by the ImageLayer object in MapObjects LT (ESRI 2000) and because of this it could not be displayed as a seamless mosaic using the imagery projected with the methods just described.

To convert or project the data properly for representation in MapObjects it would have been necessary to seamlessly mosaic or merge the imagery, project the mosaic, and then cut the imagery into tiles e.g. 1 kilometer by 1 kilometer extents based on the U.S. 
National Grid standard (Federal Geospatial Data Committee 2009) established for the Federal Emergency Management Agency (FEMA). Software tools, hardware of sufficient memory and time to do this kept it from being done thus far and projection on the fly has been sufficient. As a result, the reprojected imagery was purged and replaced by the original data when the MapObjects based interface embedded in the CBI Database diminished in importance when the emphasis of users changed. By comparison, aerial imagery for the San Francisco Peninsula simply required the completion of metadata for each image file before loading into the folder. Once both sets of aerial imagery were loaded into the folder structure, raster catalogs were created to easily symbolize and display the imagery in ArcGIS.

Charts and raster base maps were stored as navigational references for planning access to the coast by land or sea. 7.5 minute USGS DRG topographical maps were compressed to MrSID file format after all files were confirmed to be in UTM Zone 10 North NAD 83 coordinate system and projection. (At that time, other compression methods and formats were considered, however ongoing litigation over patents on wavelet compression technology and sale of companies supporting jpeg2000 or ECW software development tools in MapObjects meant that the established MrSID format was chosen.) A table of every DRG file's bounding coordinates was added to the tabular database to create a raster catalog that programmatically displayed so that the MapObjects interface could programmatically load and unload files based upon whether they would be drawn inside the extent of the map interface. Programming in this way reduced memory use and improves performance when reviewing locations at the scale of a one or two CBI segment features. NOAA nautical charts were converted from the Maptech/BSB Electronic Charts proprietary format for encrypted marine charts (.kap extension) and projected to TIFF imagery in the UTM Zone 10 North NAD 83 coordinate system and projection. The marine charts were not a seamless image set like the aerial imager and did therfore did not encounter the same problems of display when converted. All charts are stored with aerial imagery and elevation grids in the Raster sub-folder found in the CBI_GIS folder in the database.

\subsubsection{Data conversion for a flexible workflow}

PISCO staff members were not regular users of ArcGIS. Communication between PISCO staff and Pacific Coast Science and Learning Center staff during the collaboration often required the transfer of large sets of data that were in the progress of organization. Each transfer would require a complete update of up to 32 gigabytes of data of the latest version in order to continue work. This was impractical. Google Earth was seen as an interim reporting tool and planning tool that the PISCO users were familiar with. CBI Segment features that had been completed were converted and exported to the Keyhole Markup Language (as KML or KMZ) and sent to collaborators at PISCO to visualize coastline completed via email.

Segments completed were used to erase the features of the data set used as the base data for the California coastline in ArcGIS (described in the segment creation process in this document). The coastline feature with the completed features erased (trimmed to the jurisdiction of the NPS) became an estimate of coastline to be completed. By using the Google Earth files emailed back and forth, the information could be shared quickly and this enhanced productivity in trip planning. Progress reports of kilometers 
completed versus time or of kilometers completed versus kilometers remaining were easier to visualize with their GIS skill set using this method of data management.

Using aerial imagery, the California Coastal Records Project imagery and topographical maps as references with the Google Earth imagery behind the segment feature, collaborators were able to teleconference and evaluate shoreline for access and then rank the remaining work by difficulty of access. Commuting expenses and courier costs between the two office sites in Point Reyes and Santa Cruz were greatly reduced. Incremental conversions of CBI Segment data that were shared with CBI team members quickly assisted in the final correction and completion of the data collection process. Data scrubbing tasks and loading tasks became easier as the data were checked for error in small sections soon after their creation in the field rather than in large blocks weeks or months later.

\subsubsection{Subsets of larger data}

With the exception of the ESI Atlases, all data included into the CBI Database that were larger in extent than the CBI Data Extent were clipped or edited to the single feature in the CBI_DataExtent shapefile so that project hard drive space could be minimized and eventually fit on inexpensive media such as a single DVD or USB compact flash drive. The CBI Data Extent was created by the merged extent of the DEM files used to create the seamless elevation grid that was used to derive a coastline polyline that was then used to edit into CBI Segment line features. Aerial imagery was reduced to the files that only display the coast line rather than inland areas in order to significantly reduce the project's final distribution size. DRG files were loaded to match the CBI Data Extent to aid in navigation by road or trail to the coast. Boundary files were clipped to the CBI Data Extent. In addition to the reduction of the storage size of the project, limiting the data sets improved performance when used in MapObjects display or ArcMap documents by not consuming more memory or display resources than necessary.

Frustration with the existing spatial data set of the Coastal Biophysical Inventory led to an appreciation for complete metadata and led to the adoption of standards as well as the expectation for more complete documentation of methods and data used in the project. Communication about the data set and its potential use in the future assisted choices in correcting data or removing it from the system if it could not reasonably be maintained to standards or expected use. If metadata had been more complete from the beginning of the project, many scrubbing and data loading or removal tasks would have likely been less and the reiteration of undocumented processes with documented ones could have been avoided. 



\section{Chapter 7 - Implementation}

After a requirement analysis was done (described earlier) implementation of the project occurred in the following stages that paralleled the management plan sequence listed previously.

1. Software programming training

2. Pilot project programming

3. Database interface programming in VB 6

4. Database installation programming in VB 6

5. Change of project leadership

6. Evaluation of project goals and interface design

7. Documentation of methods and data collection protocol

8. Data Management and metadata standards

9. Revision of observation database design.

10. Database interface programming in Access VBA

As listed above, the stages of software programming training, pilot project programming, database interface and installation programming in VB 6, change of project leadership, evaluation of project goals and interface design, documentation of methods and data collection protocol, data management and revision of database design have been described previously in the document already in the context of the iterative design that occurred. This section will focus on the database interface programming before and after the change of project leadership. Directions to the user for database interface are included in the PowerPoint document "Coastal Biophysical Inventory 2.3 Introduction to Data Entry and Explanation of User Interface Tools” and will be limited here to avoid duplication of that document referenced in Appendix B.

\subsection{Training}

Training followed the path outlined in section 3.4.5. It culminated with independent study that resulted in the completion of the pilot project "California Hummingbird Database.” Training not outlined in the training plan included coordinating one on one consulation or tutoring.

When trying to create a repeating data control to emulate the continuous forms native to Access in order to show pictures it could not, I created two examples of a possible solution. Because the data repeating objects involved have limited documentation or examples I needed additional examples to compare to my own solutions. I submitted the project goals to use the DataRepeater object in VB 6 to an online software project management website for competitive bidding by an international collection of programmers (Exhedra Solutions 2001-2009). After reviewing several bids, two programmers were contracted and given clear specifications to negotiate a cost and deliverable. This led to two example solutions to compare to my own versions. Using the three different solutions I derived a working version of my own custom ActiveX 
object. This side project proved to be a useful project management experience in addition to the programming techniques learned.

The training plan worked rather well. The enrollment in software programming instruction, individual tutoring, and the programming a pilot example project went well and produced results implemented in the final design.These are still valuable experiences that assisted all aspects of the project. With that experience and the guidance of the revised protocol document, the programming of the final user interface required approximately the same time estimated (3 months) in the original project plan.

\subsection{Pilot Project}

Because of limited programming experience at the start of this project, the programming method followed object oriented programming goals and repurposed existing code and subroutines from other projects wherever possible. The resulting code doesn't have the unified vision of a master programmer. It can be overly simple in some places and awkwardly complex in others. While the aesthetics of the code are nothing to brag about, the fact that it does work and performs well is a testament to the effort to meet the database and interface design goals.

The programming of the pilot software project was effective. It created a product that met all the design specifications including unanticipated tasks such as developing software installation packages for deployment. The project was a MapObjects LT 2.0 based map interface programmed in VB 6 connected to a Microsoft Access database that allowed spatial selection and browsing records of hummingbirds of California and the display of imagery that was structured inside a tab based interface.

The programming prototype "Hummingbirds of California" interface allowed the selection of likely places to observe specific species of hummingbird by comparing the location chosen to the bird's nesting and range map. A subset of the data for all the species with ranges overlapping the chosen location is made and the attribute data about the birds for that location is displayed on the bird information page, including photos and song recordings for every species. This was functionally similar to the Coastal Biophysical Inventory's goal to display segment observations by selecting a segment through interaction with a computer map interface or through attribute selection from a list of species.

GIS programming challenges such as creating map interface tools, display of an automatic scale by units, real time projected unit calculations, interactive legend of data layers and a select by feature tools were demonstrated. The prototype also demonstrated the skills essential to the main project goals with database interface programming such as managing connections to a database, selection and display of records, record navigation tools, relative path linking for the display of multimedia files, and the ability to displaying reference documents.

Screen shots of the pilot project are provided to demonstrate the product that was used to estimate completion of the final database interface and the first implementation of interface design that evolved through the project life cycle that were based upon the programming design first attempted in the pilot. Figure 9 is a screen shot that illustrates the spatial query of hummingbird range by location and the six resulting species. Figure 10 illustrates an example of the display of data in the Microsoft Access Database and programmatic display of multimedia files using the information about their location 
stored in the database tables. Figure 11 illustrates the display of reference information useful to identifying the species in the field. The hummingbird interface and database was completed in approximately two weeks in September of 2004. It was successfully installed and operated on a Windows 7 Ultimate operating system in January of 2009 without alteration five years later, an unsophisticated indication that its programming was still sound.

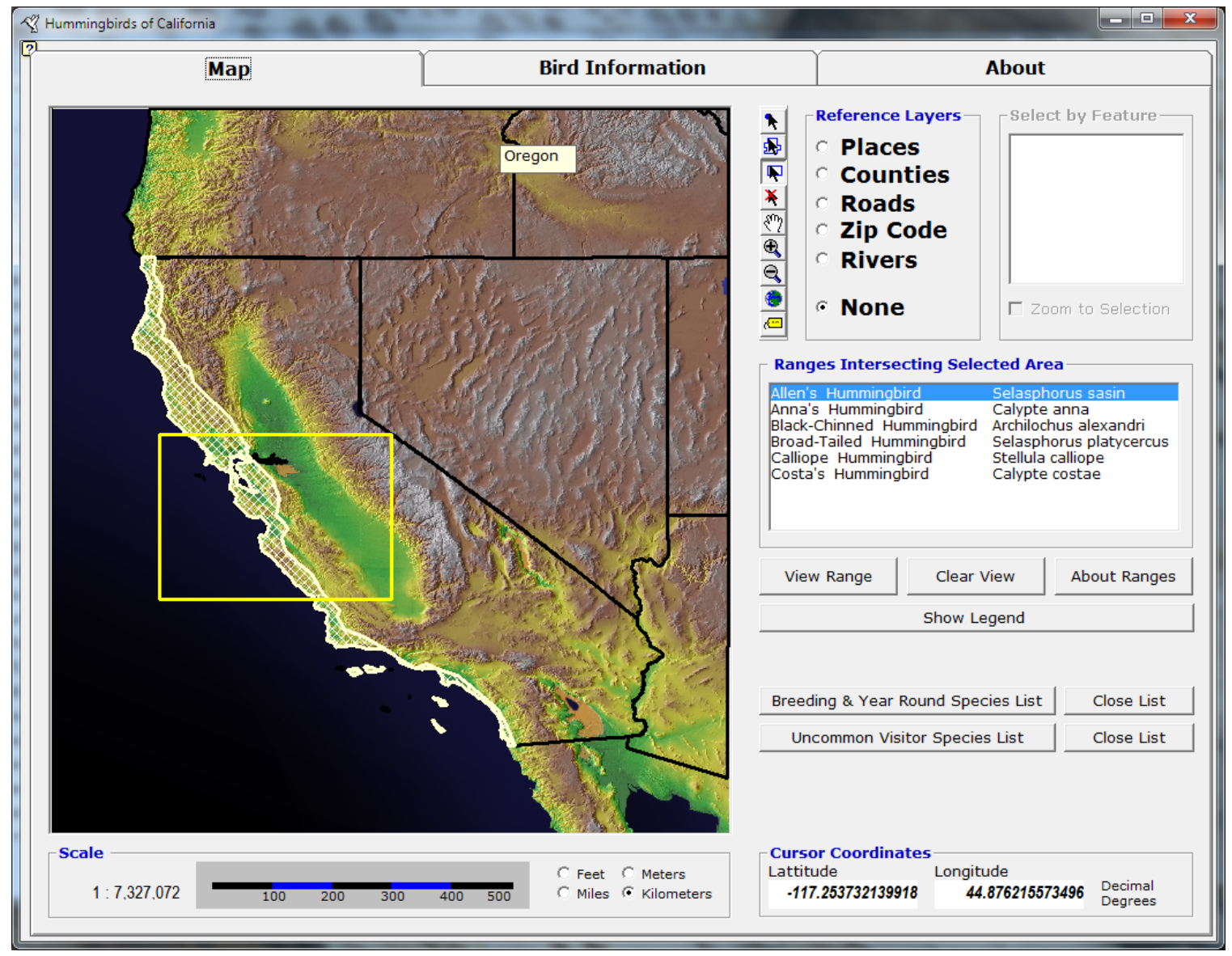

Figure 9. California Hummingbirds prototype interface screenshot, map tools 


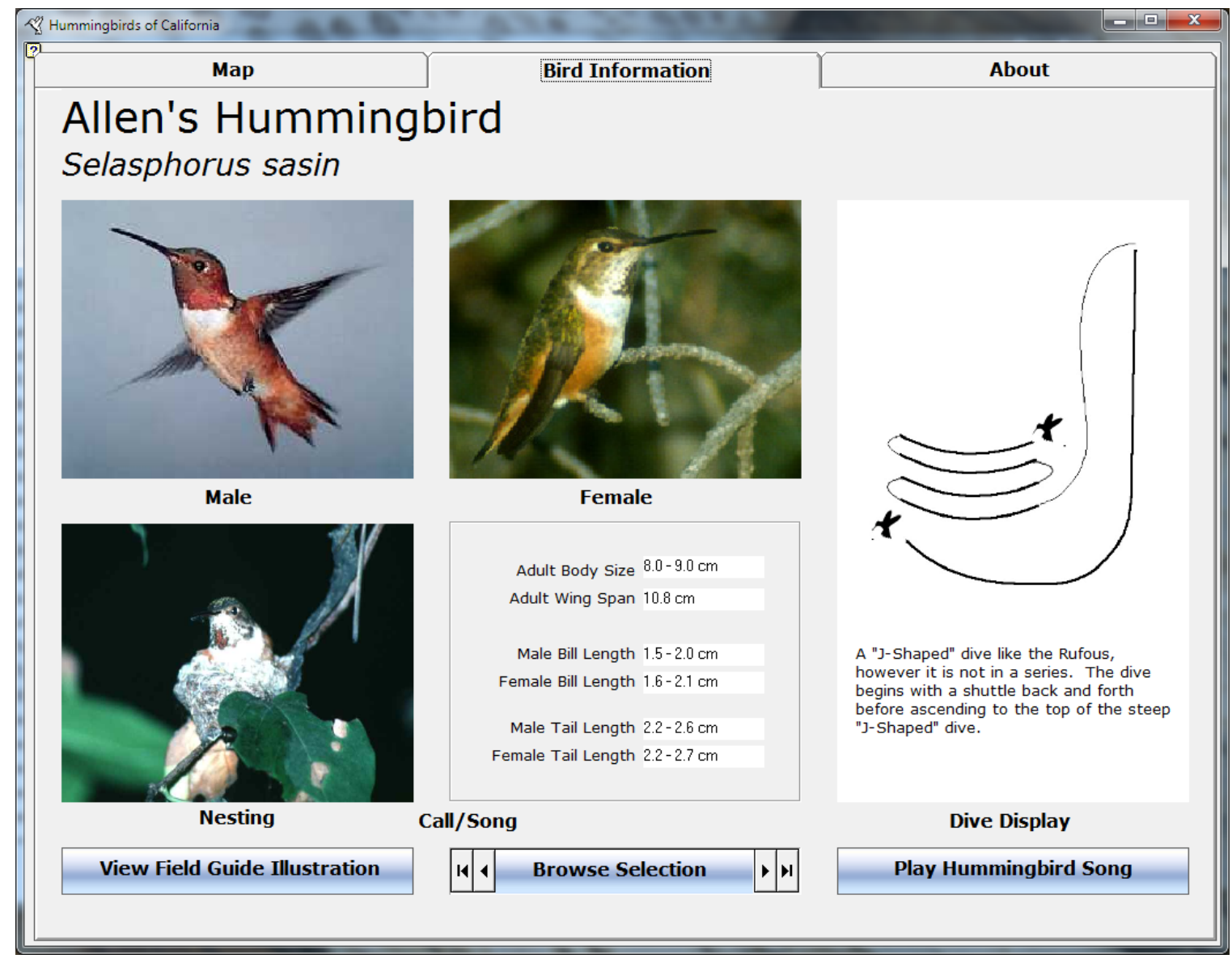

Figure 10. California Hummingbirds prototype interface screenshot, data display 


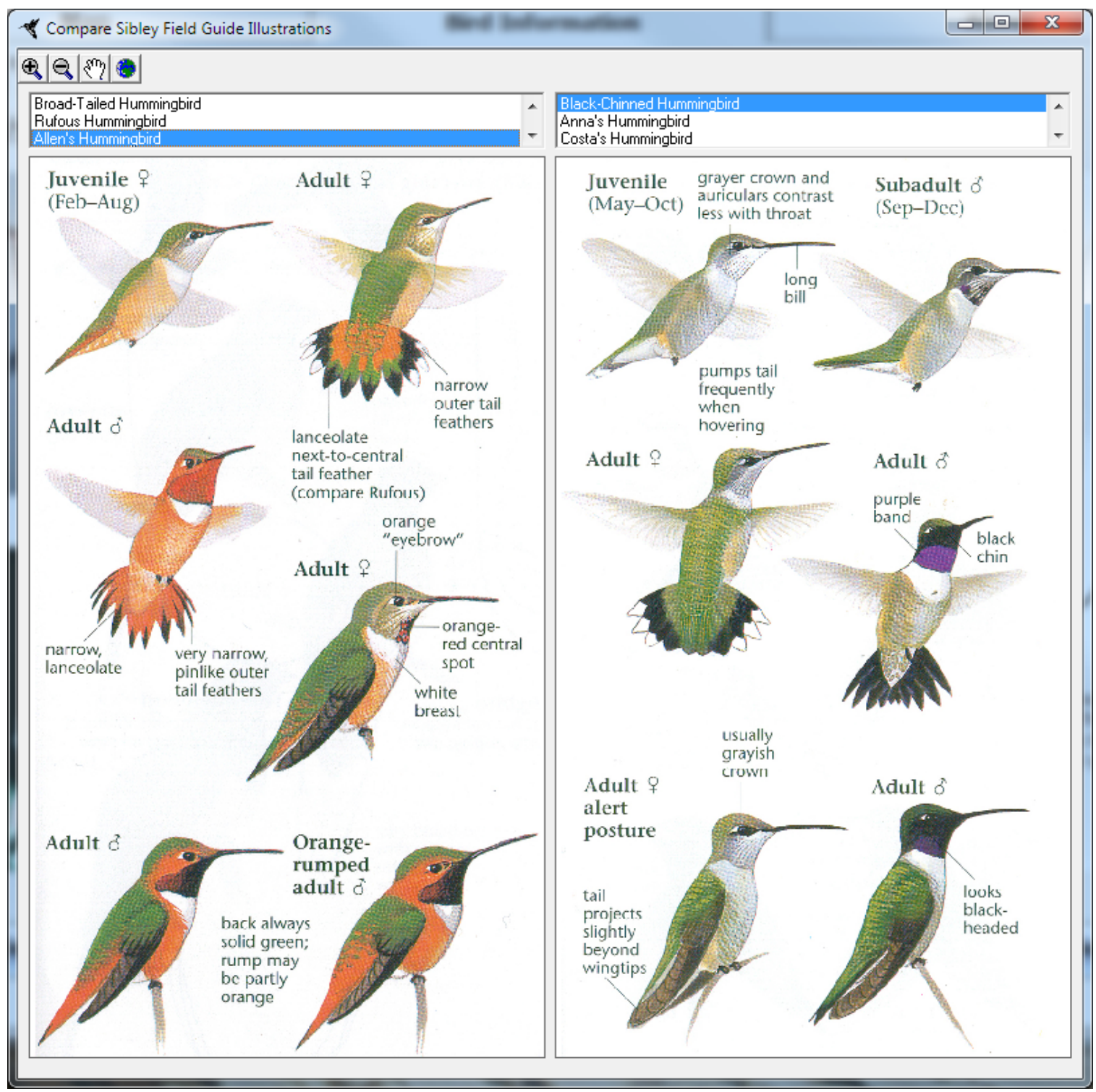

Figure 11. California Hummingbirds prototype interface screenshot, reference 


\subsection{Coastal Biophysical Inventory, Visual Basic 6 version}

Programming of the Coastal Biophysical Inventory database interface began in earnest in 2004. An interface was programmed using the existing Coastal Biophysical Inventory Database in its Access 97 format. Programming of the interface application was done in Visual Basic 6 and connections to the database were made using ADODB objects. This section will describe the evolution of that interface.

Previous versions of the database interface used continuous or repeating forms built into the Access VBA language. This was an effective way to organize data entry fields in to complement layout or data entry workflow with a design other than a row of repeating columns and rows. In VB 6 language, a custom data control was created (An ActiveX COM object) to emulate the continuous forms native to Access. This repeating data control for displaying substrate information was made using the DataRepeater object. An example of the CBI Database interface using the custom ActiveX object programmed in VB 6 is shown in figure 12. The screenshot shows the display of the multiple shoreline substrate types as one travels inland from the tide line (ocean's edge) to the terrestrial environment for each segment. Each field inside the custom control was programmed to connect to a database table to store and read data, allow another table to populate the list of choices in the drop down menu and a third related table (if needed) would store the data used to enter into the database based upon the choice made. In Figure 13 is a view of the redesigned database interface component using the repeating forms control built into Access VBA's programming environment on an Access 2007 database form. Comparison should reveal the similarity between the two results created by the differerent programming environments.

The VB 6 application that was created for the CBI Database interface will be discussed briefly to show that a working version was created, how design elements evolved from the pilot project and to give contrast to the final design solution in Access VBA that will be shown later in this document. Figures 15 through 19 represent screen shots of the CBI database interface programmed in VB 6. The figures tend to represent the view of the tabbed pages as they are selected from left to right at the top of the data entry interface display. The figures represent different displays of interface and are represented in this document at approximately one quarter of the display size of a 12 inch by 16 inch flat screen LCD monitor. The screenshots are displayed to show general layout and design evolution only and the ability to read all the text or labels in the figures is not expected.

Each screen shot represents the attempt to display a complex set of data. The interruption of the completion of this project to invest energy in a revised protocol was well worth it in order to clarify the relationships and conceptual structure of the complex data. There are no data integrity or error checking rules built into the interface. The interface design layout is similar to the final database. An important difference to notice is that it does not have an observation record viewed "inside" of a segment record. The record inside a record is a data structure that was turned into design element later. In this database interface it is difficult to tell whether to navigate the segment or the observation record and what this action does.

The design should also indicate a greater emphasis on functions built into the map display that were deprecated or replaced later. Function was replaced by connecting the 
observation database to ArcGIS projects through the OLEDB connections built into the desktop GIS software. Data used to visually overlay the CBI Segment data was readily available and built in as choices for display in the VB 6 version of the database and interface. Symbolization and labeling were also given special symbolization tools. Map query tools were not integrated before the interface and programming environment was abandoned due to change in project goals. Data entry in the first design did not progress through the interface as a simple advancement of the tab, nor did it logically follow the flow of reading the data sheet. Customized automated reporting tools built into this database version, such as exporting to Excel, did not continue to the new interface due to existing exporting tools found in Access and ArcGIS.

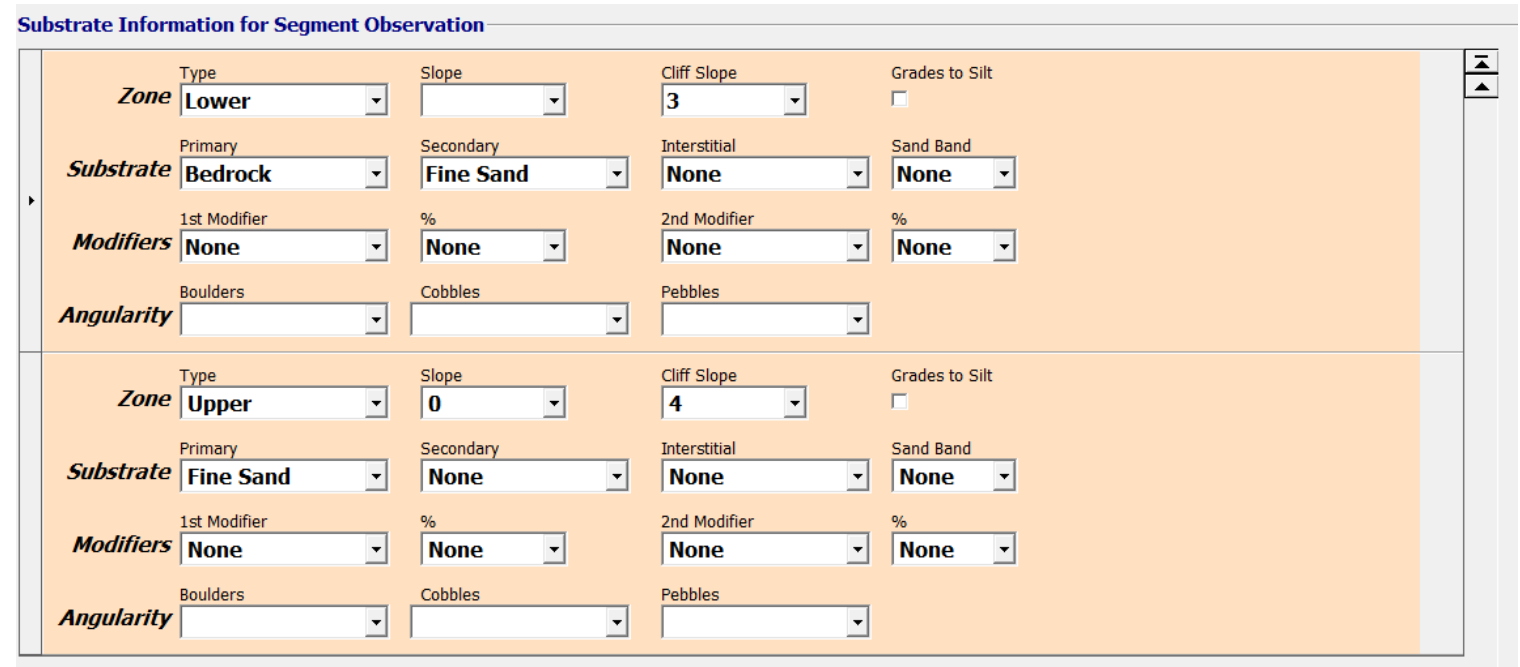

Figure 12. DataRepeater custom ActiveX object substrate display 


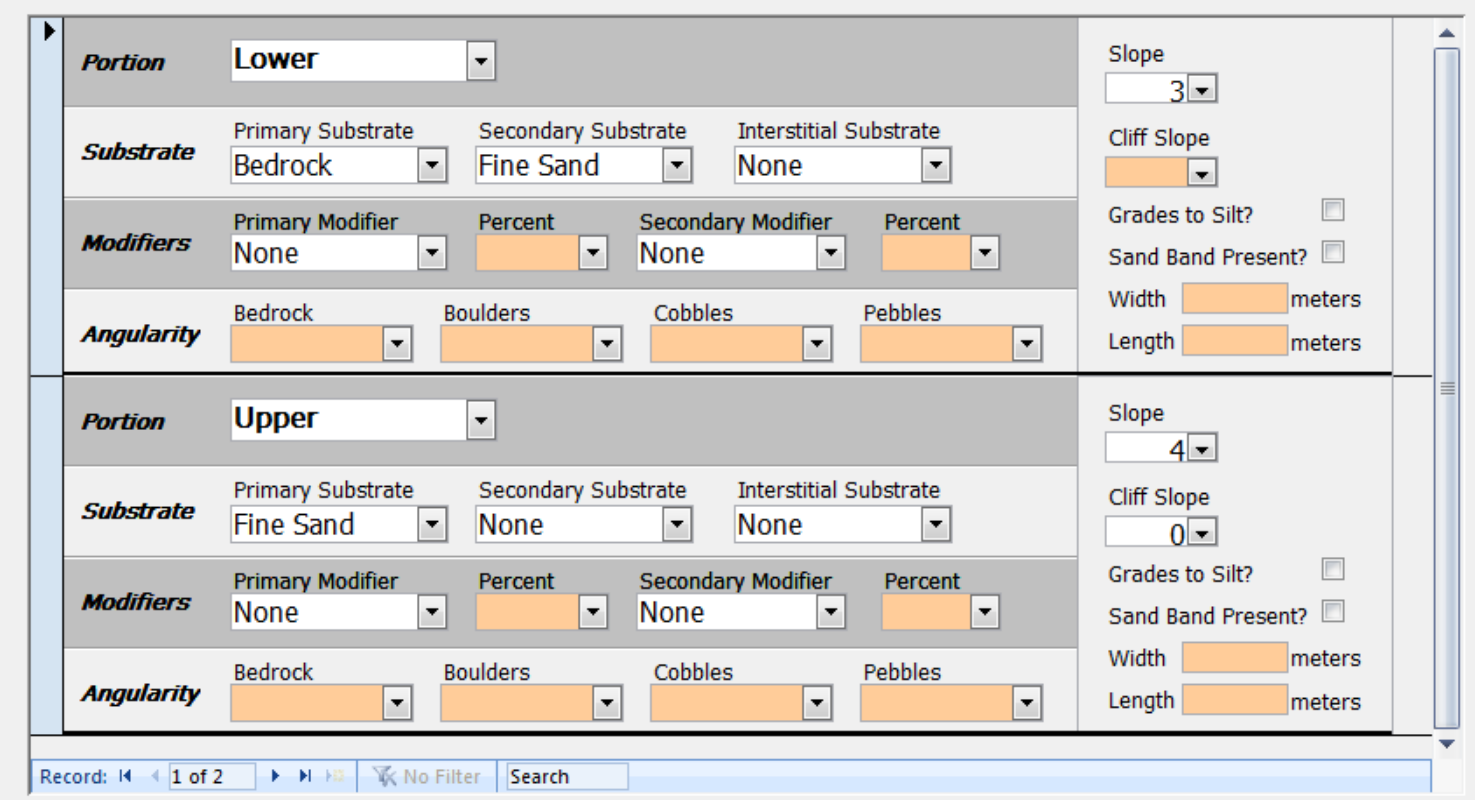

\section{Figure 13. Repeating forms display for substrate in Access VBA}

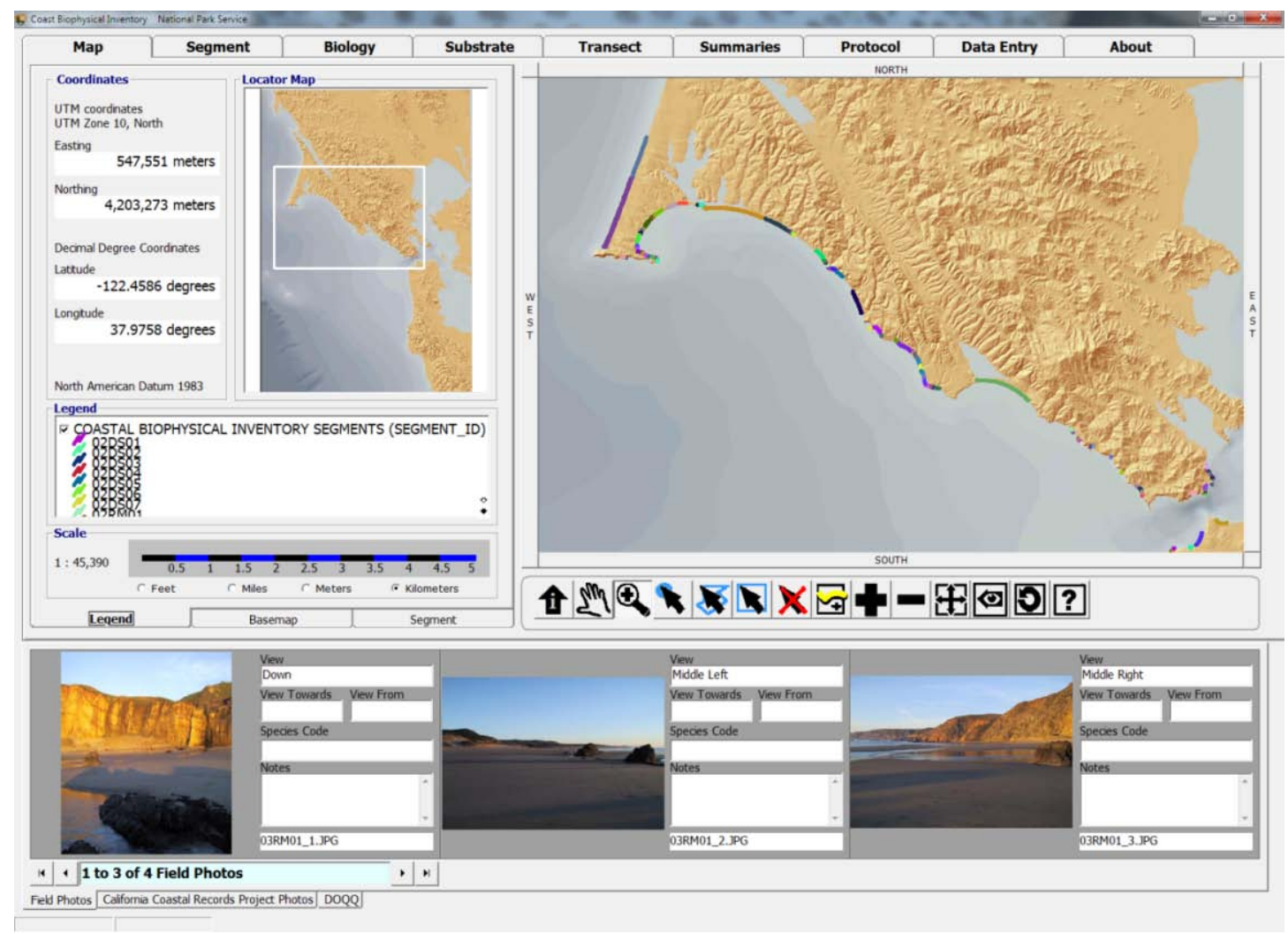

Figure 14.CBI VB6 interface, map page screenshot 


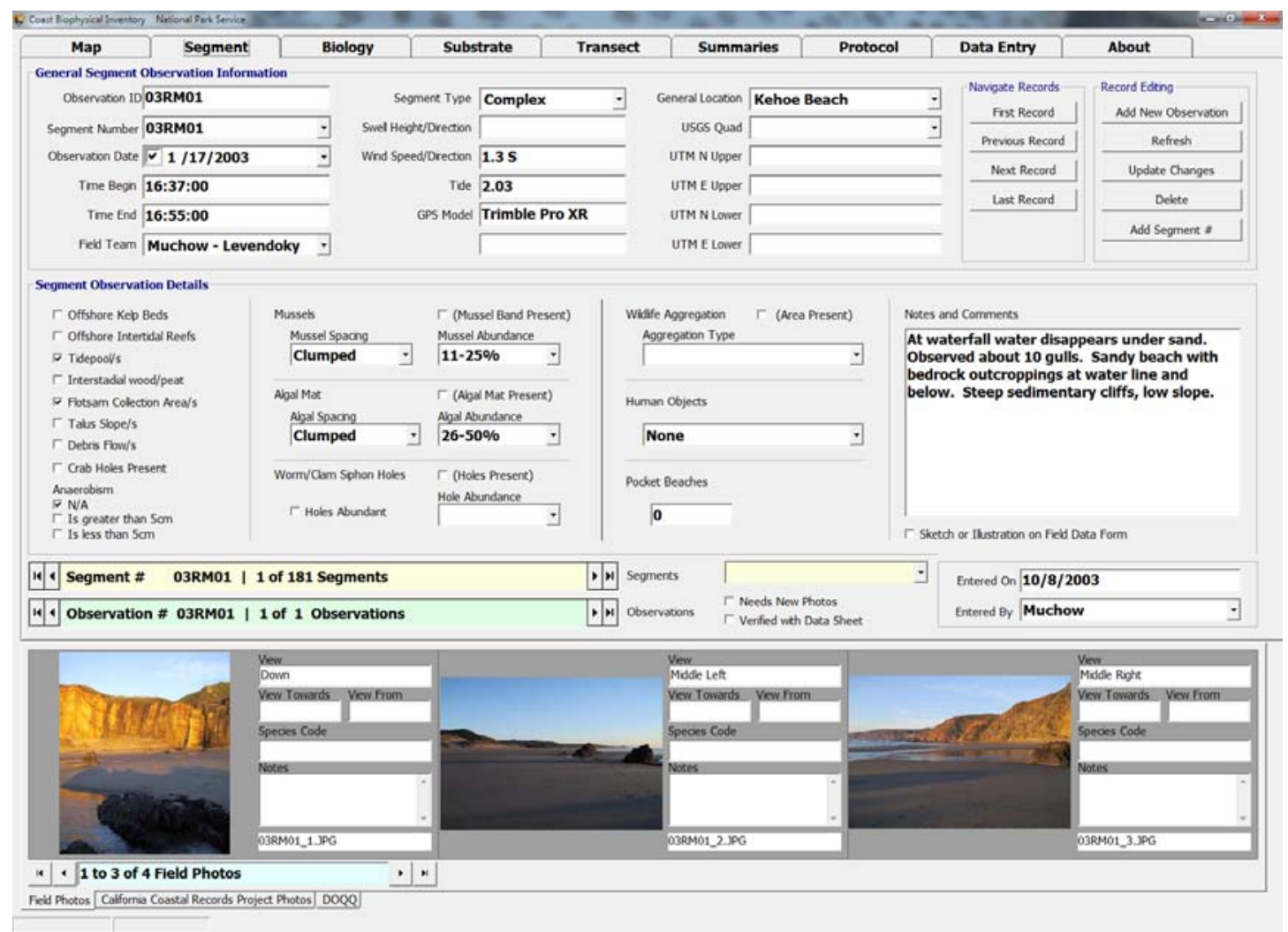

Figure 15. CBI VB6 interface, segment observation screenshot 


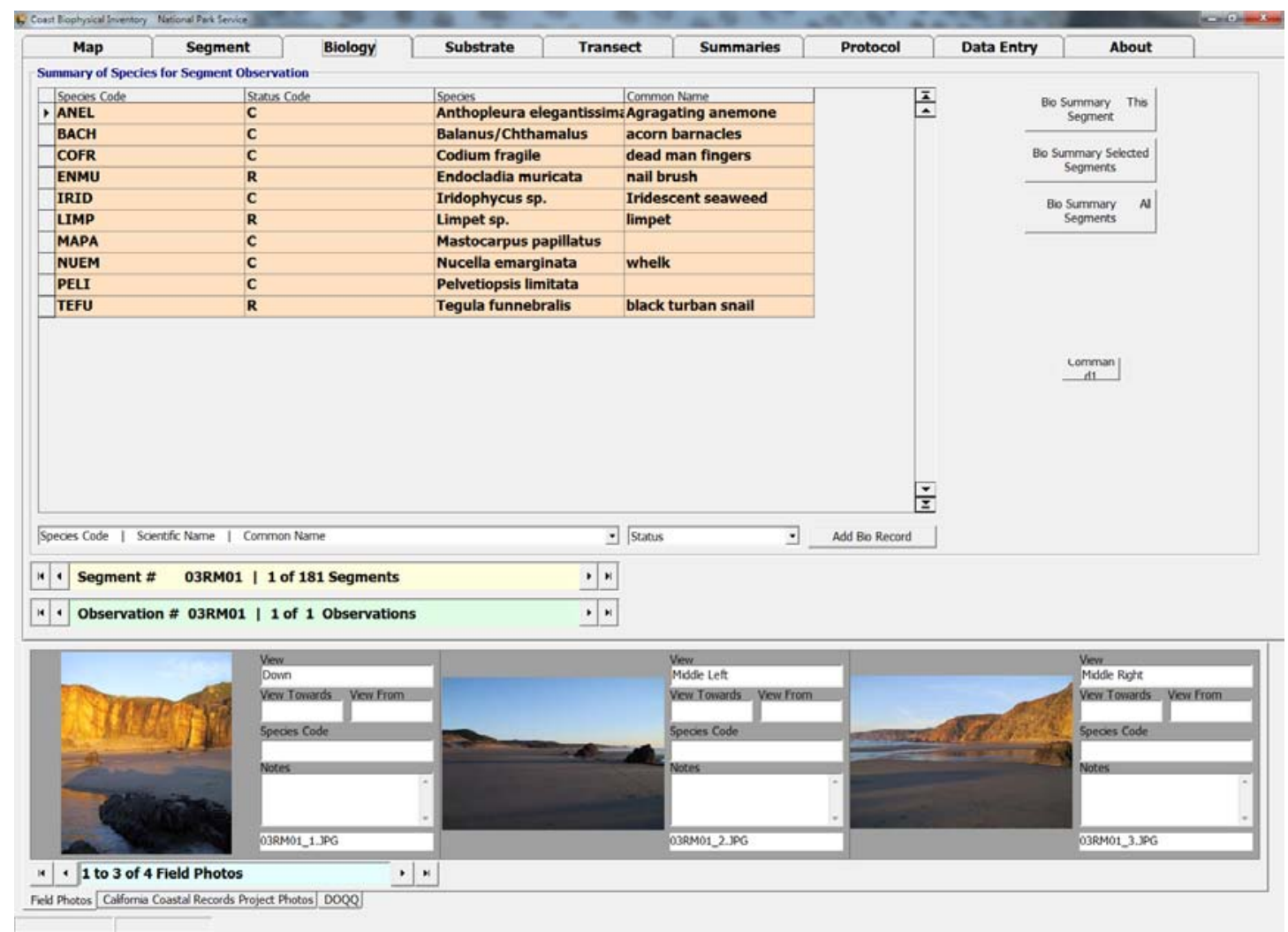

Figure 16. CBI VB6 interface, biology page screenshot 


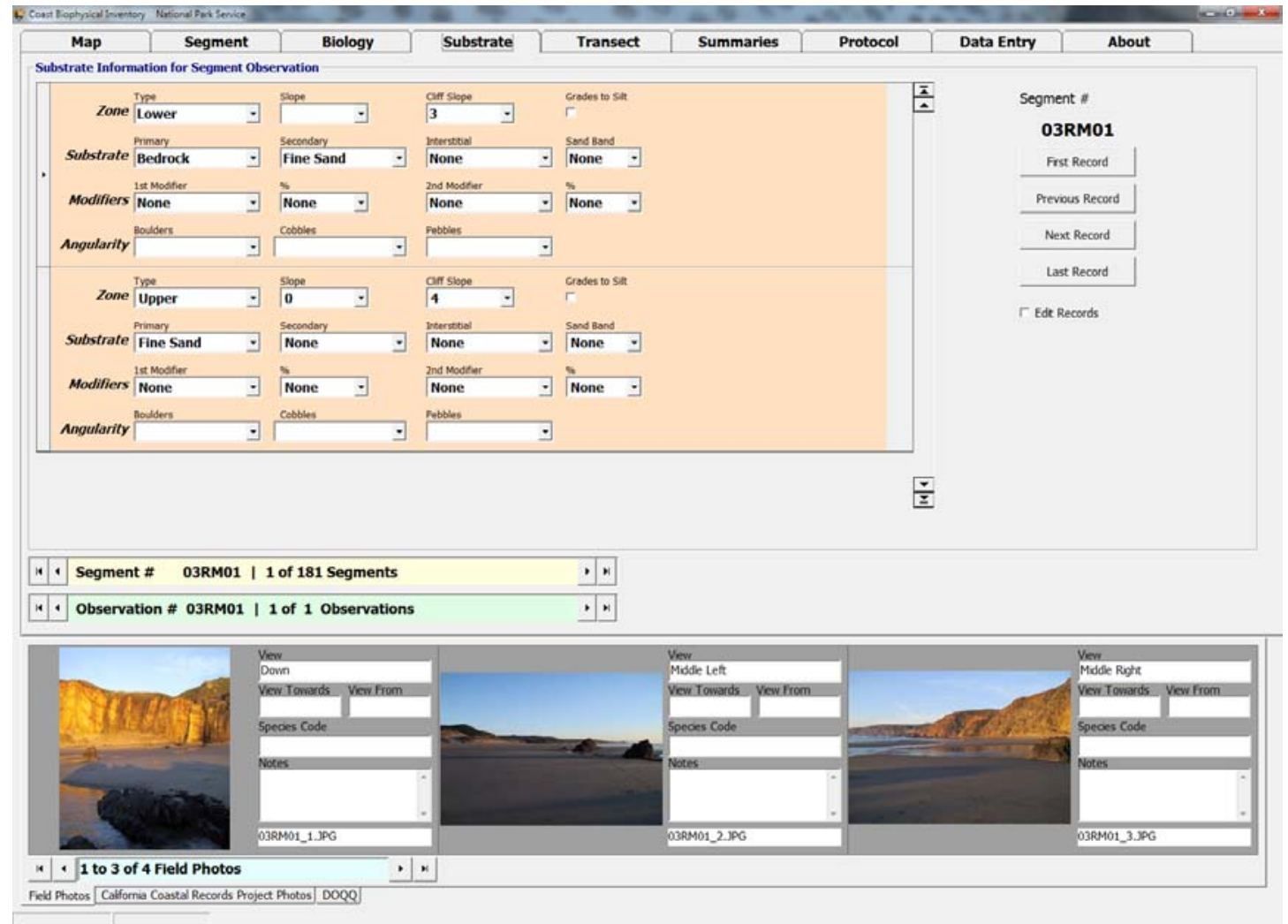

Figure 17. CBI VB6 interface, substrate page screenshot 


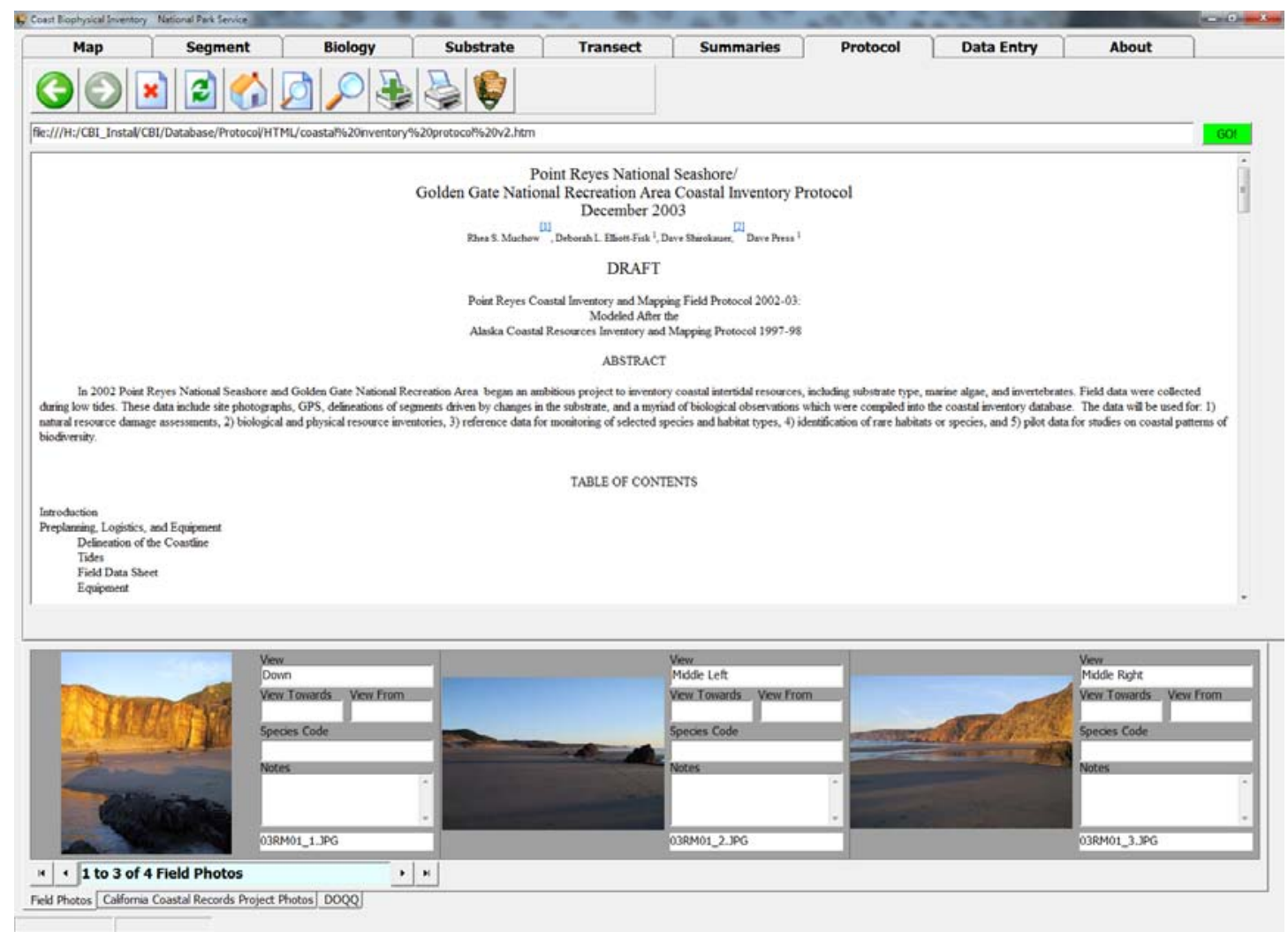

Figure 18. CBI VB6 interface, protocol and documentation page screenshot 


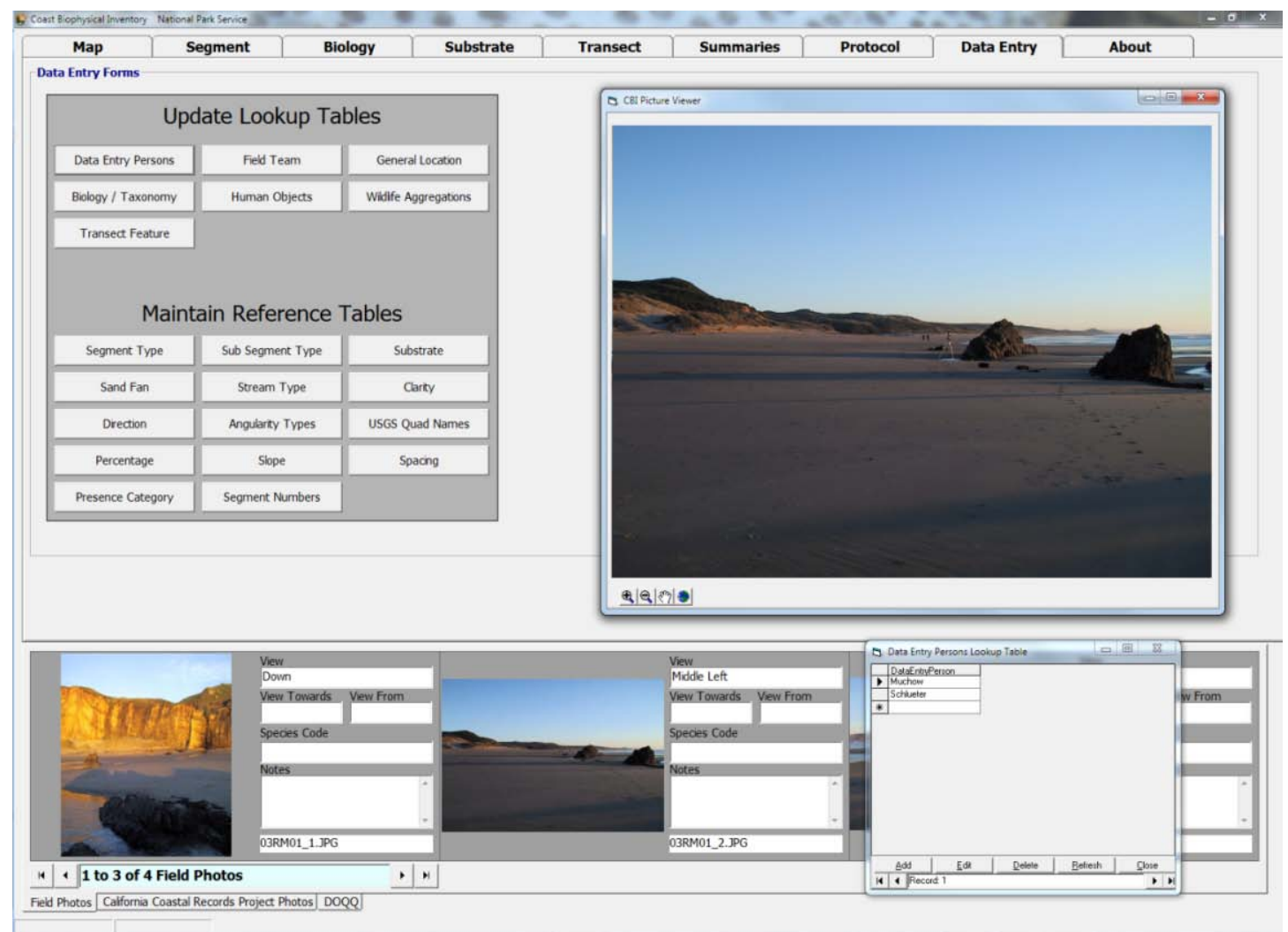

Figure 19. CBI VB6 interface, lookup table and image viewer page screenshot 


\subsection{Coastal Biophysical Inventory, Visual Basic for Application version}

The final database (version 3.0 currently in use) was programmed entirely in the Visual Basic for Applications programming environment built into Access 2003. All VBA code in the database was programmed or adapted from other code by me, except where noted in the embedded commented sections of the code when open source modules were adapted to the database. Much of the code was inspired by the work of MapObjects programmers like Jim Barry and Access VBA programmers Paul Litwin, Ken Getz, and Allen Browne.

The interface makes use of the combined database and interface programming environment available in Access VBA. It continues to use the tabbed folder interface design employed by previous versions of the database; however the nested data theme takes a visual prominence as a design motif. The emphasis on data entry and continued maintenance of data shifted the programming back to the more limited programming environment of Access VBA from Visual Basic 6, but benefited from the built in data display controls special to Access VBA.

Because the interface is compiled by the Access Application on demand when it runs, updates and improvements do not require the release of a newly compiled executable to replace the prior version. Also, the database does not need new installer software programmed to distribute the new interface version unless new ActiveX objects are distributed.

Versioning and version tracking for the software can be limited to the Access database file itself. Examples of the interface design of the final version of the database can be seen in figures 20 and 21. Figure 20 shows the first tabbed page of the observation data entry section. Figure 21 shows the map interface tools built into the observation database interface. For a complete review of pages, functions and layout see the PowerPoint document "Coastal Biophysical Inventory 2.3 Introduction to Data Entry and Explanation of User Interface and Tools” described in Appendix B.

\subsubsection{Feature requests by users, an example of software programming}

The following is an example of a feature request taken from the error log and the resulting programming solution implemented in the project. There were many requests and improvements made during testing and use to correct error or improve performance. The following example request is by Rani Gaddam and the response is by Joseph Kinyon:

"Feature Request: Currently the database is designed for auto completion of the species name, but for some species there are multiple organisms from the same long name genera. Typing out the whole genera each time is tiresome. Is there a way to just type in the species name and get the organisms, skipping the genus name?”

"Response: Currently the validation and species works well correct? There are other higher priority corrections and features to accomplish, but I have some ideas that I will try and get back to you.” (Gaddam and Kinyon 2009)

In order to get back to her in a timely way I needed to evaluate the priority of the change compared to the data entry efficiency that would result. After determining that this would encourage completion of the project and that the programming effort was within my skill set, the change was approved and the programming went forward after the problem and the function was completely defined. The effort was worthwhile and 
immediately improved the entry of about 200 remaining segment records (see data sheet for example of data set) with an average of 15 organisms to enter per segment entered, a total of $\sim 3,000$ data entry tasks.

The database combo box is located on the main data entry form under the Biology on a subform under the label "Organism". When a user types a word into the combo box, the list advances itself to the first example of those typed characters. This is already a fairly quick process and a function native to the combo box list control. During use of the combo box, the cursor advances to the section of the list with multiple genera, and the user can press the "down" arrow key to navigate to the correct one. The request was to make this section of data entry even faster for the user. The solution was to use the speed of switching the row source to enhance that control. The drawback is that for some users is that it creates a "flickering effect" that is unattractive when it is used because it requeries the source for the combo box's list of options with each keystroke.

The original row source for the combo box for species entry was connected directly to the BIO_NAME field of the Biology Look-Up table and listed all species possible alphabetically. The new code creates a subset of all records that are similar to the characters recently typed by the user. Each character added to the combo box window by the user narrows the list of options in the drop down list that is displayed. An example of the Access VBA code that implemented the feature within the drop down combo for organism selection is provided below.

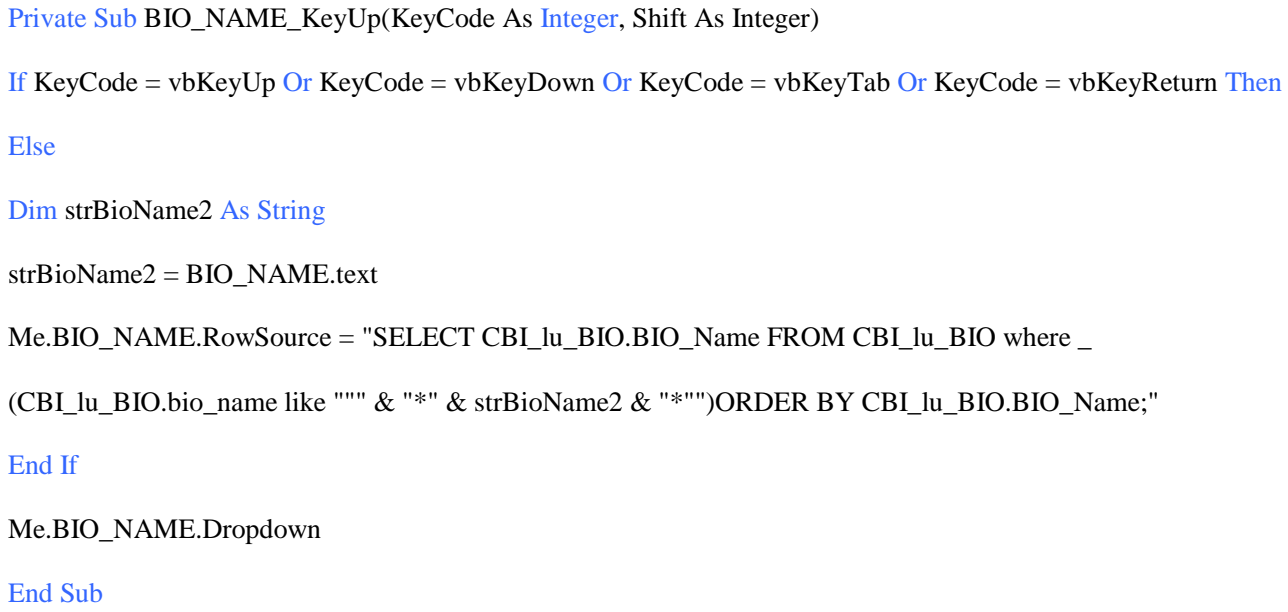

In the code above, the "keycode" variable catches the key value entered by the user which results in no action if navigation keys are pressed or (the term "Else" represents the alternative choice in the code below) it summarizes the text already entered and limits the drop down list to only organisms with those characters in that sequence in its name. If the species name is typed, it returns all organisms in any genus with those characters in its name. This was a practical solution given the domain of taxonomy limited to the CBI. It would possibly prove less useful for databases that are for a larger diversity of habitats and species.

The SQL query is dynamically created by the code with each keystroke and then assigned to the drop down list's row source. With each key stroke (as long as it isn't one of the four listed exceptions) it gives the instruction to select all the records in the biology name field found in the biology look-up table that contain the characters typed as they are 
typed and then return the matching results in alphabetical order. For the relatively short list of species used for the survey, this routine moves through the list of species names and returns the results quickly_-satisfying the function request and improving data entry. Migration of the database to the Access 2003 format and Access 2007 VBA required that this and all the code explicitly reference the version of DAO database objects, or the database would fail to run due to an ambiguity of names for the controls in the new programming environment.

The programming example is one of hundreds of subroutines embedded into the forms for displaying the user interface. The code is best understood and followed when viewed "live" using the Microsoft Visual Basic programming tool built into the Access 2003 or Access 2007 interface.

\subsubsection{Programming around error, an example of one solution}

A disappointing limitation to the continuously repeating form in the Access database interface is that it cannot display multiple images in the record set unless the data is loaded into the database as a binary large object (BLOB). Loading the imagery into a table contained in the database as a BLOB would quickly overload the database. The BLOB does not maintain the compression of the JPEG image format causing further undesirable expansion of the database and use of drive space. For this reason, the repeating form was not a useful design choice for displaying multiple images. Image record navigation and viewing occurs in the database by displaying one image at a time.

There is a design limitation to the Access record navigator where the record set navigation tools it can advance faster through the records than the images load into the form. This means that if the user advances too quickly through the records, the Access software tries to load and display the images for the records it has advanced through at the same time as the one being loaded for view and abruptly halts (crashes) the Access process. This is a documented issue with Microsoft Access and the crash behavior is exaggerated if the record changes before the "image loading" message window closes.

I anticipated that if the database attempted to load multiple images while advancing, it would crash that much more often. As cameras advanced in resolution the images created later in the project were much larger (8 MB vs. .5 MB) the increase in image size resulted in longer processing time-- therefore, as the project continued it was estimated that the system would crash would be more likely to crash as image sized increased with unknown and possibly destructive results to the existing data set. While it is possible to program multiple images to display simultaneously on a page, the user can only edit one photo record at a time therefore the faster display of only one image was chosen as the design.

The prediction of future issues with the larger image files proved true with regular crashing. It became evident that in order to move forward with the interface design the Microsoft Access VBA picture control would have to be replaced. It would have been difficult to program a replacement picture control (a fundamental control in a graphical operating system) from scratch. Alternative picture controls were sought. The "off the shelf” ActiveX control Ammara DBPix was designed to resolve the problem encountered. The redistribution license for DBPix was purchased and the control embedded into the interface for royalty free distribution. 
The use of that DBPix 2.0 ActiveX control was a costly solution $(\sim 350.00)$ relative to the additional software expense up to that point ( $\$ 1000$ for MapObjects LT 2.0). Fortunately, its anticipated use aligned with the choices and distribution solutions already made for the MapObjects LT 2.0 objects. By comparison, it would likely take several weeks to program a replacement solution from scratch and test it which would be a cost in labor several times the purchase price of the off the shelf solution. The choice to purchase the custom control was worthwhile and the images now display easily without crashing Access.

\subsubsection{VBA and VB 6, the combined solution to interface and installation}

The choice to return to the VBA programming environment for the final version of the database interface resulted in being a good one because programming database connections and queries using SQL code inside the Access database software were much easier to implement than in VB 6. When programming a few connections to view an Access Database, having a lightweight front end connecting to the database is the best choice, and installations can use the Access format database file without Microsoft Access software installed or licensing the Access runtime. The awkward arrangement of the VBA programming environment compared to Visual Basic 6 Professional or Visual Studio 6 was eclipsed by the convenience of the many database objects and query tools built into the Access software and the runtime for Access 2007 has recently been released for free, enhancing the inexpensive distribution and use of the most recent version to others.

Some VB 6 programming was still needed beyond VBA. The database requires a base level of hardware and software to operate. The use of the VBA code and the interface requires the user to install supporting software libraries and objects used by the software in its calculations and operation. The installation software created for the VB 6 version was recycled to install the objects used by the VBA software interface.

Installation of the user libraries is accomplished through a custom installer package programmed in Visual Basic 6. It is an automatic installation of the components required by the interface software such as Microsoft Data Access libraries, DBPix picture control, and ESRI MapObjects ActiveX objects. This installer saves the user from the tedious activity of installing or registering each programming library or object individually. Examples of embedding automated ActiveX installation routines into Access tables were investigated as a way to simplify distribution, but were not implemented in the current versions and are being tested for future versions.

The installation is a rather simple set of steps including copying the whole database to a location on the host computer, running the setup application, interacting with the application set up dialogue until it is finished, and running the database. The installation instructions are detailed enough to respond to most installation anomalies. The installer program is not discussed in any depth in this document and is an example of additional effort to complete the project left out of the scope of this document in order to focus on the design of the database and interface. The installer software is included on the DVD accompanying this document. 


\subsubsection{Data entry}

Tabular data is entered and maintained through the Data Entry page of the User interface. Because the Data Entry page is an Access Database, operation of the database and manipulation of the forms, tables, queries, reports and modules should be familiar to users of Access databases. A user manual was created to improve the familiarity with the interface as well as model an effective sequence of data entry tasks. Data entry is completed through three main workflows: Trimble GPS to ArcGIS, Digital Image to dataset folder, and Field Data Sheet.

\subsubsection{Data entry solutions for GPS}

The Trimble to ArcGIS workflow is discussed in length in the protocol document referenced in Appendix A. The fundamentals to this workflow are the collection of data that matches the methods of the protocol and the structure of the complementing database. For this reason, a Trimble data dictionary that was neither complex nor overly brief was created for the collection of attribute information in the field. The drop down menus and automatic stamping of features collected with required information improved speed of data entry and this dictionary worked well on the three GPS systems used, the Trimble ProXR TSC1 data logger, the Trimble GEOXT and the Trimble GEOXH. Entry of this data in the field also meant cleaner workflow when differentially correcting the points that were then converted to shapefiles. By following this work flow the resulting exported point feature shapefile continued to hold the attribute information that is essential to the processing of the coastline into Coastal Biophysical Inventory line features representing the extent of substrate types.

\subsubsection{Data entry for observation photos}

The camera images are sequentially numbered by shot. Unfortunately many times the operators did not know how to operate the time stamp and numbering features. Pushing the button is the best one can expect and correction is generally impossible after the fact. This means that as images come in from the field, they were immediately transferred from the camera, copied to a folder named for the segment they represented and then each file was renumbered based on the labeling protocol. This folder was then added to the Coastal Biophysical Inventory dataset in the proper folder structure so that during data entry the automated paths to the photos and data entry could function. Management of the imagery data is almost exclusively accomplished through the built in cutting, pasting, and renaming tools of the Windows GUI. The user can easily copy digital files from most digital cameras or media using Microsoft Windows Explorer.

\subsubsection{Data entry for tabular observation data}

Data entry of tabular data requires two sets of data records to complete, the segment log and the field data sheet. The essential data from the segment log are the names of the field team. The segment log is maintained as an archive to document effort and total project time as context to the time required for a single segment observation. This information was useful to adjust expectations of time needed to complete the inventory of 
remaining coastline. All other data is recorded on the main data sheet, on any additional biology observation data sheets and on attached notes.

The data entry technician, or user, begins by making a backup copy of the database before data entry, so that in the event of some catastrophic shut down or interruption that corrupts the database, all previous data will be preserved. The next step is to open the database and open the data entry page. Once on the data entry page, the user advances the page to the segment for which the observation is recorded. When the correct segment is the current record, the user inserts an observation record and begins manually transferring data to the database through transcription.

Starting at the top of the data sheet, as the data entry technician reads from top to bottom, they use the tab key to advance to the next control of the observation record on the user interface and enter the data from the data sheet. In two places the user is required to browse and interactively enter data; those are the location of the digitized version of the original data sheet and the locations of the related photo records for the observation.

While there is a single undo function available in Access 2003 and later, the user is manipulating the data "live" without any extra validation or checking beyond those for each control. Many users are used to seeing "submit" buttons with feedback screens that highlight corrections used in web based data interfaces. This is a preferred method for simplified data entry, however this design was compromised to satisfy the other design goal to browse, to enter or to correct data after entry.

The data entry follows the nested design structure of the data. Once at the desired segment record, the user opens and inserts a new observation record. The user then can insert into the new obersvation record associations to related photos, biological information, scanned data sheets and substrate information. The database user can close the data at any time and it is saved. When it is saved or closed, the database runs through the default automatic compaction and repair routine to maintain the database at its optimum size and form. Detailed and annotated screen shots of all functions with step by step instructions and the corresponding section of the paper data sheet are in the data entry manual. In figures 20 and 21, screen shots of the observations section of the database and the selection tools are provided as examples of the interface design as it manifested in the final design when viewed in Access 2007.

\subsubsection{GIS data in ArcGIS}

When the licensing available to the NPS was expand to install ArcGIS on all compatible computers in the agency nationwide, the programming for a custom MapObjects base GIS interface with overlay and analysis tools was evaluated as duplicative of the tools available in ArcGIS. The effort to continue the development of those custom spatial query tools built with MapObjects was deprecatedfor a new interface with the bulk of editing and analysis performed using the tools in the ArcGIS software interface.

The CBI Segment data and the first database interface built in VB 6 did not meet new user needs for planning new field work due to the inability to calculate, track and report the coastline yet to be inventoried,. It also could not report the new task of how much work was remaining completed compared to the previously completed inventorya regular part of project management and milestone tracking. Finally, MapObjects LT 
2.0 could not calculate or be programmed to perform the regular editing and display tasks expected of the CBI Segments while the collection of new data was underway.

Management of the GIS dataset in the Personal Geodatabase and the related folder structure is done through ArcCatalog and ArcGIS. While it is an important part of the function of this project and integral to the final deployment of the Coastal Biophysical Inventory, it is beyond the scope of this report to describe the operation of the GIS beyond the data management description and workflow listed in the protocol. The used of ArcGIS is not significantly specialized beyond the CBI Segment data storage structure described already and can be accessed like many of the introductory tutorial examples for ArcGIS.

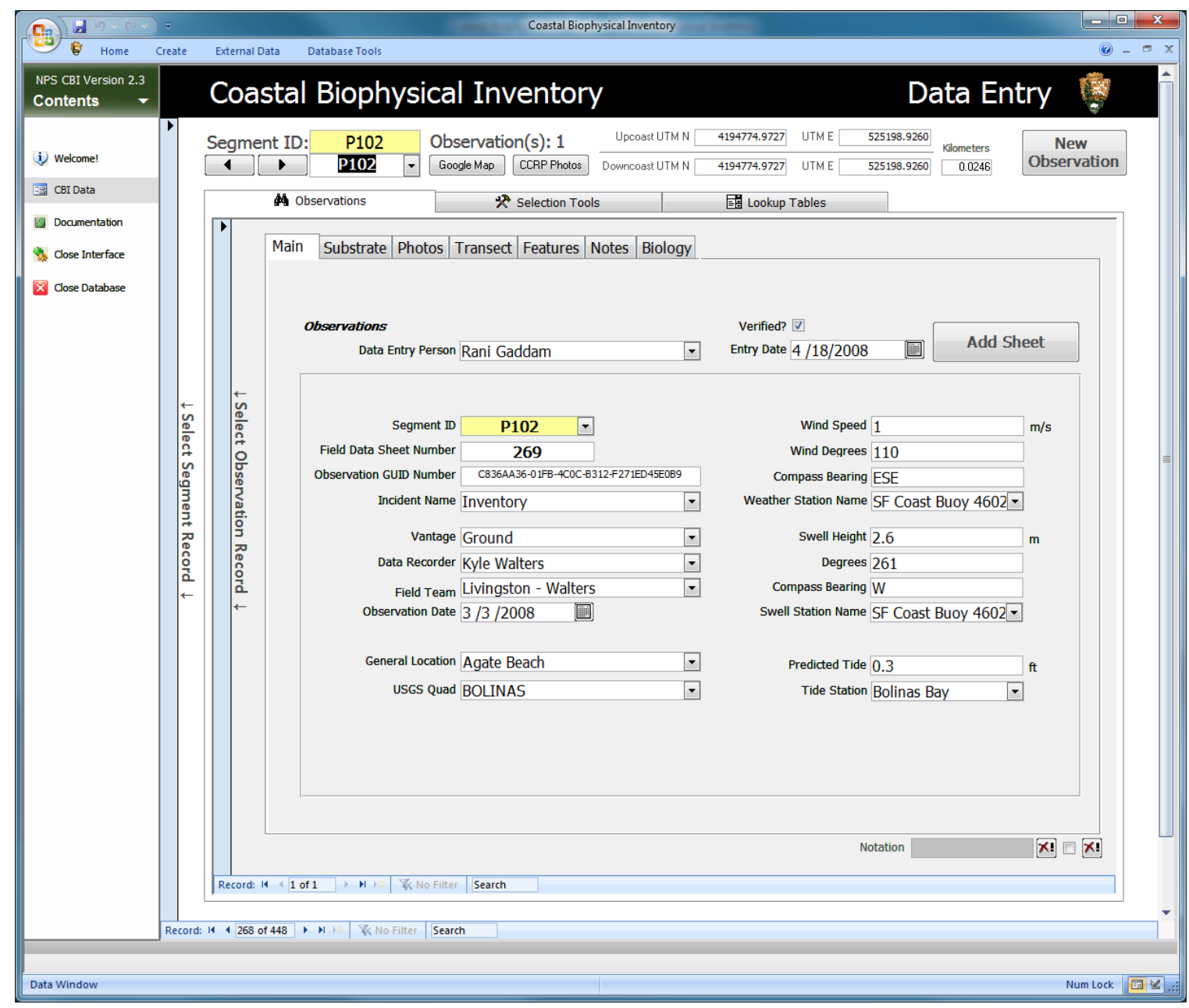

Figure 20. CBI database interface, Access VBA version screen shot 1 


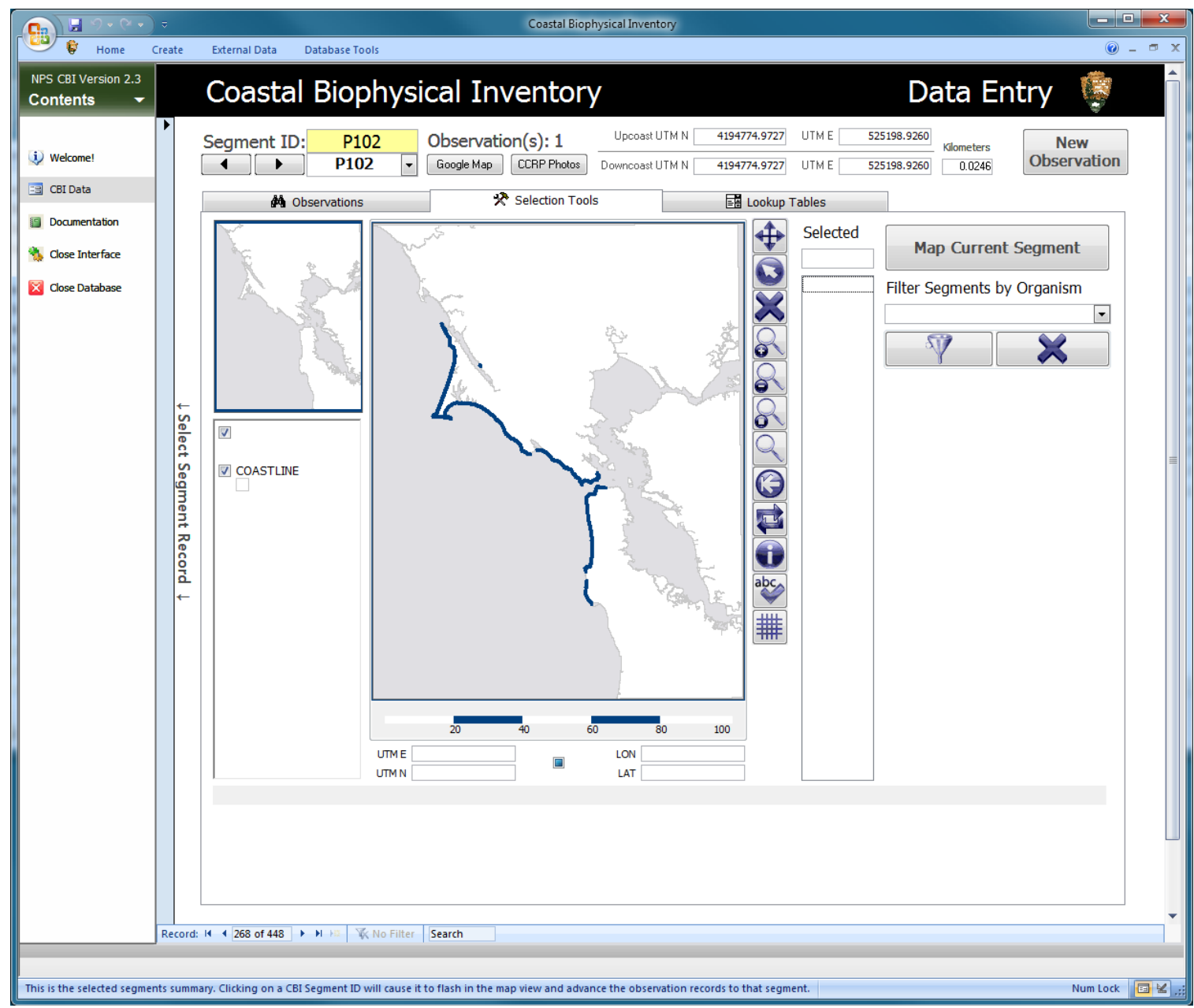

Figure 21. CBI database interface, Access VBA version screen shot 2 


\section{Chapter 8 - Results and Analysis}

The completion of the CBI Database and interface allowed the completion of the mapping of approximately 161 kilometers of the intertidal zone between Tomales Point and Pillar Point and it represents an excellent baseline of data for coastal resource managers as planned. The development of protocol documentation that delayed then facilitated its completion is essential for any repeatability in the future. The programming is easily accessible and maintained through Microsoft Access. The database and interface met many of the goals anticipated.

The database functions as both a data entry tool and as a data viewer where the user can browse and select observation data as anticipated in the planned designed. Resource managers can quickly browse information about coastline they are familiar with and perceive how the database complements or reinforces their understanding. They can also review coastline they have not visited to plan additional field observations or to create a sense of an unfamiliar location. Species observations by location can quickly be summarized using ArcGIS. Locations that include an observation of a specific organism can be queried and displayed in one click using the MapObjects base map interface or through more complicated analysis using customized Access queries or connection to ArcGIS.

The data set and interface were received and accepted by the Department of Fish and Game as completed deliverables for the grant money given to the project. The project was invited to be presented at the California Department of Fish and Games Oil Spill Prevention and Response annual symposium of research projects. The project was one of the last (due to financial crisis in the State's budget) oil spill research projects funded by the California DFG and will hopefully serve as an example to reinstate that grant program. Public presentation to this agency helped make other groups aware of its existence and the potential for the experience to assist new intertidal damage assessment programs.

The design and experience gained from this project has been used to represent the National Park Service in data acquisition planning meetings in anticipation of California's Marine Life Protected Areas public input process, NOAA's Natural Resource and Damage Assessment workshops and by biologists collaborating with the Mineral Management Service’s Multi Agency Rocky Intertidal Network (MARINe) oil spill response planning. The experience of assembling reference data identified the special gaps in intertidal data that many coastal resource managers struggle to overcome. The problem of not having high quality coastline information at the extent of the CBI coastline at a scale larger than 1:24000 is a continuing one. The lessons learned from assembling the GIS data helped prioritize the effort to acquire LiDAR data for the coastline.

Using ArcGIS, the observation data stored in the Access database was connected to the CBI Segments feature class. Through this connection, a summary of observations such as the location of rocky shoreline and diversity of organisms observed per segment was created and reported as maps to the California DFG to articulate its capabilities. See Appendix D for a suite of example maps. These summaries are valuable as tools to communicate the value of the existing data and other potential use. 
The most significant result to the change in the database is that the changes to the database's design to include unplanned observations have been successful. The shift of the database design from representing one observation for one segment to the repeatability of many observations for one segment recod means that the data can be a framework for other observations along the coast that benefit to being related to substrate or location. The database and protocol did not originally include assessments of abundance, but can accommodate that extension of use in its revised design. A contract to extend the database to include abundance measurements for mussel beds at each segment began in December of 2009. Recycled modules of VBA code, database structure, and table relationships resulted in version 2.3 being being adapted to include the data from this ongoing project in approximately 4 days (including testing) and resulted in version 3.0 of the database and interface.

Statistical analysis of the completed observation data set was made to determine confidence in the data set. Conclusions from the analysis of biodiversity data indicated that the data are affected by survey team even though great efforts were made to normalize data and methods. The whole data set can still be useful for broad species richness assessments and understanding the baselines of minimum diversity as anticipated (Becker, Gaddam and Kinyon 2008). See Appendix E for the full analysis document.

Programming, data management efficiencies, GPS management and the management of a data collection project are all experience and insight that improves productivity daily at the Pacific Coast Science and Learning Center. Perseverance with creating a suite of protocol documentation for the project eventually turned the project around. Creation of metadata protocols continues to enhance the quality of all data managed at the Pacific Coast Science and Learning Center and will hopefully assist the transition of data to enterprise geodatabase management in the future.

I am a plodding writer and find it difficult to wrestle the many components of a project of this size into a summary document, therefore the ability to create quality documentation quickly is difficult. This experience has made me learn that documentation takes much longer than expected and the time allotted to complete documentation should be appropriately padded to allow completion on time. Once it existed, clear documentation of the protocol made the design process and the implementation process easier.

During the development of the Coast Biophysical Inventory Database, the product life cycle for the MapObjects LT 2.0 software and the Visual Studio 6 software entered the end of their maintenance stages and were later deprecated to promote newer standards. This is a risk that is part of late adoption of established programming languages or software. The ability to continue the use of the interface with the tools created and anticipated use by the existing newer GIS was seen as a condition that was best solved through the storage of data (and in some cases redundant storage of the data) in different database formats that anticipate use by other GIS software than the custom interface developed using MapObjects for this project.

While only a small part of the current database, MapObjects LT 2.0 is such a straightforward set of objects and elegantly simple programming object model, it is a shame to see it at the end of its product cycle. It would be a great boon to programmers if an equally light weight object model that subsets the functionality of the ArcObjects 
product to meet the special niche that MapObjects has filled for over a decade. I truly enjoyed working with this minimalist set of GIS programming tools. After evaluating possible replacements to MapObjects LT 2.0, the clearest potential replacement would be the open source and royalty free MapWindow ActiveX map interface control developed by the Idaho State University Geospatial Software Lab (Idaho State University Geospatial Software Lab 2009).

Testing was ongoing for this project. The original database converted from the Glacier Bay National Park and Preserve's Coastwalkers database was created in Access 97 running on a Windows 2000 operating system. Recent testing of the CBI in its current form in an Access 2003 format database on a Windows 732 bit operating system inside Microsoft Access 2010 revealed its viability for approximately five more years in unaltered form. All screen shots in this document were taken using Access 2007 operating in Windows 7. The VBA code was updated to explicitly reference Access data connections and doing this allowed the successful conversion of the database form Access 2003 .mdb format to Access 2010 .accdb format, should it be needed. It was amazing to see the challenges of data migration for a project this small over the five years it occurred. This suggests that if the pace of database design or software continues to change rapidly, then important data sets run the risk of being lost because of obsolescence and deprecated interoperability.

While not explicitly addressed in this document, the size and scope of the work, the many alterations to the primary goals and the length of time to completion should indicate that it was a project that suffered under scope creep. Some of that scope creep could have been contained by me, other parts could not. When you invest yourself into a project like this and have signed on to the end, the project gets done. In some cases this can ruin people, especially if after all the effort the project fails or resources simply run out. The resource for me that ran out long ago was Time, but the project continues on as an active dataset maintained for ongoing data entry.

I left the Masters of Science program before finishing the MIP for GIS job that was rare in a location that was exactly where I wanted and needed to be. I knew the consequences could result in delaying my MIP as it has, but I didn't know how great that risk really was. When, through a turn of events, I became my own client at the very same job I had left the MS GIS program to pursue. The dilemma of being manager of the project intensified the negative consequences of not completing the MIP. In order to complete the tasks of my job and complete an oversized MIP morphing into a full blown research project, I often worked sixty to seventy hour weeks to meet both the goals of the project and the MIP. The lessons learned are too numerous to illustrate here. In fact I would have done almost everything different with the hindsight I have gained. However that is too brief a summary and I will enumerate the highlights of my hindsight:

- Scope creep is a constant and regular battle in a GIS project. Team or employ people who manage the timeline if you are too busy to do so. Together you will achieve excellence.

- Working full time and completing a MIP required a dedication of time that can create health risks, most notably is the increased risk of repetitive stress injury or physical inactivity. The combination of both full time work and a MIP should be avoided due for this reason alone. 
- The Coastal Biophysical Inventory is a project of huge scope. I have received endless amounts of hands on experience seeing where small projects have succeeded or failed. I have been able to witness the full development cycle of a piece of GIS software from its conceptualization to its retirement including the collection of the data used to populate the database it uses.

- The canned data of GIS lessons will never prepare you for the monumental data management that is really needed nor for the shameful lack of metadata in real world projects.

- Software, software, software. Learning new software and methods is a constantly essential task to plan with a large project. During this project I have used ArcView 3.3, MapObjects, ArcGIS 8.3 - 9.3.1, Erdas Imagine 8.7, GE Tools, XTools, Visual Basic 6, Visual Basic for Applications, GeoExpress, ER Mapper, Trimble Pathfinder Office, Trimble Terrasync, Windows XP, Windows 7, Windows Mobile 2003, 2005, \& 2008, Thumbsplus, Microsoft Office 97, XP, 2003, \& 2007, Google Earth, Picasa, and Photoshop. The list goes on. The tools, tricks and solutions to get the job done are apparently endless.

- Confirm your protocol is complete and clear before you sign on create a database for any research project. If the protocol is still morphing, then the science isn't being applied as diligently as it should. The database design and the data that is collected will benefit greatly from a well documented protocol

- A well designed database will help everything else fall into place.

- A user interface cannot be really exceptional for complex software without significant improvements to operating systems. There are too many existing obstacles to this goal. You can only make powerful short leaps towards it in a project. 


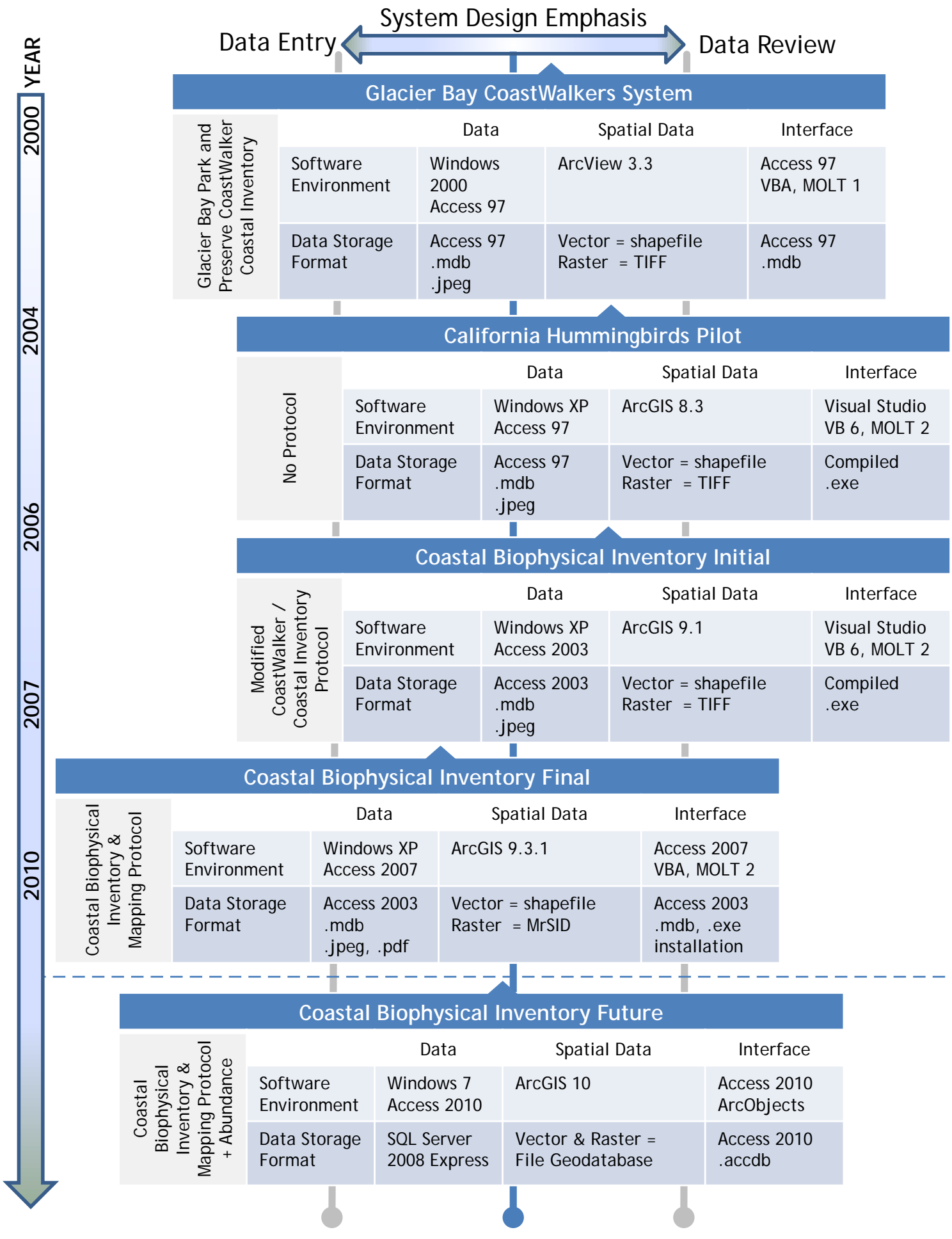

Figure 22. Comparison of system versions and interface design emphasis 


\section{Chapter 9 - Conclusions and Future Work}

The goal to create a map interface to a Microsoft Access database was met. The route to reaching that goal was circuitous. An extensive amount of work not discussed here was part of meeting milestones that were contingencies for the project's completion especially the creation of better documentation. Documentation of data collection methods and project standards were extremely useful and should be obtained prior to commitment to work on similar projects. A change order process for database design will have a hard time accommodating a developing research method and all database design should be considered an evolving construction in the future even with fixed data collection methods.

\subsection{New and unexpected uses for the CBI}

While the resulting database and interface is a large effort to take pride in, it is always my sincerest hope that this database is never used for the problem that inspired its creation an oil spill. However, during the inventory an oil spill event occurred. This was distracting to the schedule for the completion of the project, but also tested its use beyond the hypothetical. Post spill damage assessment and questions about the project from other groups indicated some surprising uses of the data as well as revisions to understanding how damage assessment is done.

Some sections of the coastline are treacherous and walking those sections of the coast is impossible and required a revision to the protocol. The easiest to access locations had already been done and the logistically most difficult to access sections remained. The low hanging fruit had already been plucked; therefore estimates of effort to complete the remainder were in error.

By interpreting photos and USGS maps, the remaining coastline left to inventory was ranked by difficulty to access. This map gave us an idea of how to prioritize our efforts and where the greatest challenges were. In November of 2007 the cargo container ship the M/V Cosco Busan struck the San Francisco Oakland Bay Bridge and the bridge fender penetrated the hull and fuel tank causing the release of approximately 53,500 gallons of bunker fuel. Currents carried surface borne oil outside the entrance to San Francisco Bay and oiled beaches inventoried by the CBI. After the spill happened it was surprising that after collecting all the biophysical data about the coastline, it was an interim map about the beaches ranked by difficulty of access created for CBI project management that was the piece of data first requested

Because it is impossible to consistently identify the thresholds for different intertidal zones on the always differing substrate, a clear marking of the thresholds for these sub zones was never created in the transect observation protocol. Some organisms mark the beginning or end of intertidal sub zones on some substrate, and when these organisms occurred they were recorded on both the biology section of the datasheet and their distance from the low tide zone was recorded. Because the transect marked the upper end of the intertidal zone, it essentially defines the width of the intertidal zone. Multiplying this by the length of the segment, one can derive the area of the intertidal zone and interpolate the biological observations across it. 
In the future, Natural Resource Damage Assessment (NRDA, an oil spill incident command role) would like to know the location and area of the subzones represented within the transect data.. Because zonation was not recorded for each segment, the data cannot provide this estimate for a damage assessment team. However, if elevation above sea level is used, one can calculate the width of the slope of the beach if one knows the angle of that slope. Because the angle of the slope was measured for each segment, one can attempt to calculate area using the transect width and angle of incline for the substrate. Because current map data for the extent of the Coastal Biophysical Inventory is at a scale and resolution that has an error larger than the entire intertidal habitat, the current techniques offer the best assessment of zonation until elevation data with horizontal and vertical error of less than 30 centimeters or less $\left(\sim 1 / 10^{\text {th }}\right.$ the annual tidal elevation extremes) is created for the entire extent of the intertidal zone at the location studied.

At the end of April of 2008, a researcher from the University of California working on a type of isopod that lives in sea caves wanted to identify the potential location of its specific habitat in the Point Reyes National Seashore. Because sea caves are a special coastal feature, they are always recorded in the notes (indicating in hindsight that they should have been explicitly noted as a feature in the Protocol from the beginning). An interim map of sea cave locations and was completed and this was used to prioritize field efforts by the researcher. Similarly, when black abalone was added to the federal endangered species list, a map of observations and likely habitat was summarized for further investigation.

It is impossible to predict all the future uses, but unfortunately the most likely use will be for an oil spill. In the recent Cosco Busan event, the valiant but miserable deployment of inadequate oil spill booms indicated that the oil spill response in this area is underfunded, poorly supported by the industry and cannot prevent the damage to the coastline that this database's information might prioritize for protection. Most of the areas north of the Golden Gate benefited by the luck of the weather moving currents the oil away at that time. I am certain our luck will not hold out and the regularity of large oil spills worldwide and locally indicates that we will eventually be turning to the data stored in this database for a before and after comparison of catastrophic damage.

\subsection{Anticipated evolution of the dataset}

The ability to communicate the results of the CBI to others is the greatest improvement of this data. The ongoing success of the data will be the summarization and reporting of the data. This can be done through a variety of formats.

One of the most successful exports of the data has been the creation of Google Earth KML/KMZ place marker files. The quality of aerial imagery and the quality of its navigation interface make Google Earth an effective way to illustrate the location of data or data summaries that allows the user to quickly get a sense of place and conditions without the hurdles of learning the customized software. The export of the complete database to a Google Earth file as a report is anticipated in its design and will be the next phase of the data's development.

The database currently sits in an Access database to facilitate data entry and portability. The future of this database might be to convert it to a Microsoft SQL Server 
database and create web browser based user interfaces to view and browse the data. Web based applications are a current development focus, along with open source programming tools. This environment was considered for the database, but the ability to use or move the data if access to network resources were gone was seen as a higher priority.

Another improvement to the database would be to derive a higher resolution coastline from LiDAR elevation data and snip the segments from this higher resolution data. This higher resolution coastline will help estimate the resources needed to continue further research of this type as well as give estimates of areas of damage. It would also build a stronger spatial data set to build future observations upon.

Through the design of the user interface and the database, this project is optimized for data entry. An important enhancement to the user interface might be to denormalize the data into large tables that allow quick browsing through simple text controls rather than the current combo box data controls required for data entry. Another solution is to index every item in the database for Online Analytical Processing Databases (OLAP) and their enhanced data mining tools. The ongoing migration of the data from a database without data integrity logic to one that has cascading updates and enhanced logic stored in its structure is essential to its continued use. The need to correct data that is poorly organized or full of idiosyncrasies or misspellings defies even the most sophisticated data analysis tool or optimization.

Because loading the images to view is a significant performance issue, it might be useful to make small thumbnail images of all the field photos and embed them as blobs into an adjacent database optimized for this task. Loading these embedded thumbnails is much faster and investigations into this could be accomplished through the batch resizing and loading of the thumbnail imagery. Thumbnail images have already been attempted as a way to improve the display of the linked images; however there was no noticeable improvement to the speed of navigation through the records beyond the improvements already made to image display.

Finally, converting this to an Access 2007 format database and investigating the improved programming environment will enhance its ability to be used in the future. Access databases can still use the Database Active Objects (DAO) from Access 97. They still use Visual Basic for Applications, but its parallel professional development twin in Visual Basic 6 has been unsupported by Microsoft for approximately four years at this time. Because Access has not left the DAO environment for the Microsoft ActiveX Data Objects Database object libraries announced almost ten years ago, the only way to truly upgrade the database is to get it out of Access eventually. Getting this database migrated to a different database version will help staircase its survival through incremental upgrades of its format and help ensure its compatibility with the next wave of 64-bit operating systems.

Through the tasks of updating and upgrading the database and its interface, the clearest conclusion for this database, and possibly all databases, is the continuous migration of the database and interface to new storage formats and data management systems is its survival. An almost biological and viral conclusion is that databases need to be able to evolve and replicate to maintain their presence and usefulness. The majority of uses anticipated for this database in the future seem to require it. 
It was enlightening to see that the problems of data interoperability appeared to maintain or increase the problems of obsolescence or inaccessibility of data during the project's life cycle. The problems of not having standards that originally challenged the project's success seem to now be replaced by another problem of competing standards inside the constant versioning of database software and operating systems. Survival of the fittest may not always be the motivating force behind standards adoption as other economic or forces behind decisions to adopt a standard prevail. The greatest skill a new database developer can learn from this project is that iterative design management and migration plans should be learned early so that the management of the project can identify the cycles it chooses to oversee. Project management terminology and methods are often borrowed from the construction of discretely bounded objects. A database designer should learn iterative project management from the beginning in order to artificially create the beginning and end points for when they will steward the database and its design instead of learning how to manage database projects with tidy beginnings and ends. 


\section{Works Cited}

Adelman, Kenneth, and Gabrielle Adelman. California Coastal Records Project -- Aerial Photographs of the California Coastline. 2002-2009. http://www.californiacoastline.org/ (accessed December 9, 2009).

Alaska Department of Fish and Game. "Map of the Exxon Valdez Oil Spill." Exxon Valdez Oil Spill Trustee Council. 1993.

http://www.evostc.state.ak.us/facts/spillmap.cfm (accessed December 09, 2009).

Alaska Oil Spill Commission, the State of Alaska. Exxon Valdez Oil Spill Trustee Council; Oil Spill Facts, Details about the Accident. February 1990.

http://www.evostc.state.ak.us/facts/details.cfm (accessed December 9, 2009).

Allegretti, Thomas, et al. "Maritime Cabotage Task Force - Update on Efforts to Block Future Waivers." Maritime Cabotage Task Force. October 14, 2005. http://www.mctf.com/letter101705a.shtml (accessed December 9, 2009).

Ammara Digital Image Solutions. Image Control for Photo Database and IMage Management - DBPix. 2008. http://www.ammara.com/ (accessed December 9, 2009).

The National Parks: America's Best Idea. Directed by Ken Burns. Performed by Adam Arkin, Philip Bosco, Kevin Conway, Peter Coyote and Andy Garcia. 2009.

Becker, Ben, Rani Gaddam, and Joseph Kinyon. Exploratory analysis to examine robustness of CBI dataset: Impacts of tide, survey and season. Analysis, Point Reyes: Pacific Coast Science and Learning Center, 2008.

Berry, Helen D., R. John Harper, Jr., Tom F. Mumford, Betty E. Bookheim, Amy T. Sewell, and Linda J. Tamayo. The Washington State ShoreZone Inventory User's Manual. Protocol, Olympia: Washington State Department of Natural Resources Nearshore Habitat Program, 2001.

Burke, Robert. Getting to Know ArcObjects; Programming ArcGIS with VBA. Redlands: ESRI Press, 2003.

California Resources Agency Geospatial Information Office. California Spatial Information Library (CaSIL). 2009.

http://casil.ucdavis.edu/casil/imageryBaseMapsLandCover/imagery/urban_imager y/0.3meter_urban_area/ (accessed December 9, 2009).

Cardoza, Patricia, Theresa Hennig, Graham Seach, and Armen Stein. Access 2003 VBA Programmers Reference. Indianapolis: Wiley Publishing Inc., 2004.

Carlos, Juan, and Mariano Rajoy Brey. "SPAIN BAND SINGLE HULL TANKERS Intertanko." Intertanko. October 24, 2002.

http://www.intertanko.com/templates/Page.aspx?id=35992 (accessed December 9, 2009).

Coastal and Ocean Resources Inc. Shore-Zone Mapping of the Outer Kenai Coast, Alaska. Project Summary, Anchorage: The Exxon Valdez Oil Spill Trustee Council, 2003, 70.

Cochran, Ian (editor). "Hang on in there." TANKER Operator, October 2009: 4-5.

Committee on Commerce, Science and Transportation, United States Senate, 108th Congress, First Session. "PHASE-OUT OF SINGLE-HULL TANK VESSELS." United States Government Printing Office. January 9, 2003. 
http://www.gpo.gov/fdsys/pkg/CHRG-108shrg842/html/CHRG-108shrg842.htm (accessed December 8, 2009).

Cox, P., and G. Alemanno. "REGULATION (EC) No 1726/2003 OF THE EUROPEAN PARLIAMENT AND OF THE COUNCIL of 22 July 2003 amending Regulation (EC) No 417/2002 on the accelerated phasing-in of double-hull or equivalent." Official Journal of the European Union (English) 249 (July 2003): 1-4.

Dalby, Craig. "Telephone conversation with Craig Dalby, NPS GIS Program Manager, March 2003." 2003.

Department of the Interior, Office of Environmental Policy and Complianc. "Pollution Response and Natural Resource Trusteeship Training Module on NRDA for Personnel Involved In Emergency Response to Oil Spills and Hazardous Substance Release Under the National Oil and Hazardous Substances Pollution Contingency Plan." U.S. Department of the Interior - Office of Environmental Policy and Compliance . http://www.doi.gov/oepc/response/a01.htm (accessed December 09, 2009).

Devanney, Jack. The Tankship Tromedy: The Impending Disasters in Tankers. Tarvernier, Florida: CTX (Center for Tankship Excellence) Press, 2006.

Duncan, Dayton, and Ken Burns. The National Parks: America's Best Idea. New York: Knopf Publishing, 2009.

ESRI. ESRI Support Center -- MapObjects -- Windows Edition. http://support.esri.com/index.cfm?fa=software.filteredGateway\&PID=21 (accessed December 9, 2009).

—. Getting Started with Map Objects LT 2.0. Redlands: ESRI Press, 2000.

-. "MapObjects LT 2 ActiveX Control and ActiveX Automation Objects Object Model." molt_dgm.pdf. 2000.

http://www.esri.com/library/brochures/pdfs/molt_dgm.pdf (accessed December 9, 2009).

—. MapObjects LT 2.0. Redlands: ESRI Press, 2003.

EUROPA. Europa- Press Releases - Single-oil tankers banned from Eruopean ports from 21 October 2003. European Commission. 10 21, 2003. http://europa.eu/rapid/pressReleasesAction.do?reference=IP/03/1421\&format=HT ML\&aged=1\&language=EN\&guiLanguage=en\#file.tmp_Ref_1 (accessed December 9, 2009).

Exhedra Solutions. Rent A Coder: How Software Gets Done -- Home of the world's largest number of completed software projects. 2001-2009.

http://www.rentacoder.com/RentACoder/DotNet/default.aspx (accessed December 9, 2009).

Experts Exchange. Experts Exchange - the \#1 resource on the web for solving technology problems. 1996-2009. http://www.experts-exchange.com/ (accessed December 9, 2009).

Exxon Valdez Oil Spill Trustee Council. Legacy of an Oil Spill, 20 years after Exxon Valdez; Exxon Valdez Oil Spill Trustee Council 2009 Status Report. Status Report, Anchorage, AK: Exxon Valdez Oil Spill Council, 2009.

Federal Geospatial Data Committee. United States National Grid -- Federal Geospatial Data Committee. May 28, 2009. http://www.fgdc.gov/usng (accessed December 2009, 2009). 
Freeman, Mike. "Nassco completes oil tanker 11 months ahead of schedule - Business SignOnSanDiego.com." SIGN ON SAN DIEGO, the San Diego Union-Tribune. December 4, 2009. http://www.signonsandiego.com/news/2009/dec/04/nasscocompletes-oil-tanker-11-months-ahead-schedu/ (accessed December 9, 2009).

Gaddam, Rani, and Joseph Kinyon. "'Error log, Coastal Biophysical Inventory, Excel Document, 2007-2009"." Point Reyes, 2009.

Goodman, Sally, and Joseph Kinyon. Metadata Standards and Guidelines for the Pacific Coast Science and Learning Center. Point Reyes, August 2006.

Graves, Brad. "NASSCO eyes pact on new oil tankers." San Diego Business Journal, January 2003.

Hamil, David L. "Your Mission, Should You Choose To Accept It: Project Management Excellence - by David Hamil, Mesa Solutions." GeoCommunity. 2002. http://spatialnews.geocomm.com/features/mesa1/hamil1.pd (accessed December 9, 2009).

Hayes, Brian. Isys Information Architects Inc. Interface Hall of Shame - Tabbed Dialogs (archived version). 2000.

http://www.interfacehallofshame.eu/www.iarchitect.com/mdesign.htm (accessed December 9, 2009).

Hooson, David (editor). Geography and National Identity. New York: Wiley-Blackwell, 1994.

Hostettler, Frances D., Keith A. Kvenvolden, Robert J. Rosenbauer, and Jeffrey W. Short. "Aspects of the Exxon Valdez Oil Spill -- A Forensic Study and a Toxics Controversy." U.S. Geological Survey Toxic Substances Hydrology Program-Proceeding of the Technical Meeting Charleston South Carolina March 8-12, 1999 --Vlume 2 of 3 --Contamination of Hydrologic Systems and Related Ecosystems, Water Resources Investigation Report. Charleston, SC: U.S. Geological Survey, 1999.

Howes, Don. "BC Biophysical shore-zone mapping system - a systematic approach to characterize coastal habitats in the Pacific Northwest." Proceedings of the 2001 Puget Sound Research Conference. Seattle : Puget Sound Partnership, 2001. 11.

Idaho State University Geospatial Software Lab. MapWindow Open Source GIS . November 24, 2009. http://www.mapwindow.org/ (accessed December 9, 2009).

IEEE. "IEEE Recommended Practice for Software Requirements Specifications 8301998." Welcome to IEEE Xplore 2.0. 1998.

http://ieeexplore.ieee.org/xpl/freeabs_all.jsp?tp=\&arnumber=720574\&isnumber= 15571 (accessed December 9, 2009).

Integrated Taxonomic Information System. Integrated Taxonomic Information System. December 9, 2009. http://www.itis.gov/index.html (accessed December 9, 2009).

Kaufeld, John. Access 2002 for Dummies. New York: Hungry Minds Inc., 2001.

Kinyon, Joseph. Coastal Biophysical Inventory 2.3 Introduction to Data Entry and Explanation of User Interface Tools. Point Reyes, September 2009.

Kinyon, Joseph Guy, and Rani Gaddam. Coastal Biophysical Inventory (CB) and Mapping Protocol. Protocol, Point Reyes : Pacific Coast Science and Learning Center, 2009.

Kinyon, Joseph. Methods for processing Coastal Biophysical Inventory (CBI) field coordinates into line features of the coast.”. Point Reyes, March 2008. 
Kinyon, Joseph. Relating Acces to ArcGIS. Point Reyes, January 2009.

Litwin, Paul, Ken Getz, and Mike Gunderloy. Access 2002 Desktop Developer's Handbook. Alameda: Sybex Inc., 2001.

MarinMap. MarinMap. 2007-2009. http://mmgis.marinmap.org/dnn4/Default.aspx (accessed December 9, 2009).

McCammon, Molly. "Management of Economic and Environmental Recovery: The Exxon Valdez Oil Spill Trustee Council." Economic, Social and Environmental Effects of the Prestige Spill. Santiago, Spain: Consell do Cultura Galega and the Association of Natural Resource and Environment Economics, 2003. 1-20.

McDowell Group. An Assessment of the Impact of the Exxon Valdez Oil Spill on the Alaska Tourism Industry. Initial Assessmennt, Seatte, WA: Preston, Thorgrimson, Shidler, Gates and Ellis, 1990.

McIntosh, John. "Feedback to MIP Background Paper by Joseph Kinyon." Feeedback, 2003.

McPhee, John. Assembling California. West New York: Farrar, Straus and Giroux, 1994.

Microsoft. Microsoft Visual Basic 6.0 Programmer's Guide. Redmond: Microsoft Press, 1998.

Mitchell, Tyler. How do you bridge the CLI vs. GUI gap in app design? July 17, 2004. http://blogs.oreilly.com/digitalmedia/2004/07/how-do-you-bridge-the-cli-vsg.html (accessed December 9, 2009).

Monterey Bay National Marine Sanctuary. "Socioeconomic Uses - Section V." Monterey Bay National Marine Sanctuary Site Characterization. February 24, 1999. http://montereybay.noaa.gov/sitechar/soci5.html (accessed December 9 2009).

National Park Service. The National Park Service -- Our Mission. http://www.nps.gov/legacy/mission.html (accessed December 9, 2009).

Natural Resource GIS, National Park Service. NRGIS Program - Metadata. November 30, 2006. http://science.nature.nps.gov/NRGIS/metadata.aspx (accessed December 9, 2009).

Natural Resources Defense Council. Oil Spills - The Green Gate from NRDC. 2001. http://www.nrdc.org/greengate/wildlife/oilv.asp (accessed December 9, 2009).

NOAA National Ocean Service Office of Response and Restoration. "ESI Data in Geodatabase Format | Responding to Oil Spills | NOAA's National Ocean Service Office of Response and Restoration." National Oceanic and Atmospheric Administration. April 7, 2009. http://response.restoration.noaa.gov/topic_subtopic_entry.php?RECORD_KEY\% 28entry_subtopic_topic\%29=entry_id,subtopic_id,topic_id\&entry_id\%28entry_s ubtopic_topic\%29=463\&subtopic_id\%28entry_subtopic_topic\%29=8\&topic_id\% 28entry_subtopic_topic\%29=1 (accessed December 9, 2009).

NOAA/ Hazardous Materials Response and Assessment Division. "Oil Spill Case Histories, 1967-1991; Summaries of Significant U.S. and International Spills." Office of Response and Restoration, NOAA's National Ocean Service. September 1992. http://response.restoration.noaa.gov/book_shelf/26_spilldb.pdf (accessed December 9, 2009).

Novalis, Susan, and Dana Jones. Access 2002 VBA Handbook. Alameda: Sybex, 2001. Oil Spill Prevention and Response Technical Advisory Committee, California Department of Fish and Game. Oil Spill Prevention and Response Technical 
Advisory Committee (TAC) Meeting Minutes July 21, 2009. Minutes, Sacramento, CA: California Department of Fish and Game, 2009.

Ott, Riki. Not One Drop: Betrayal and Courage in the Wake of the Exxon Valdez Oil Spill. White River Junction, VT: Chelsea Green Publishing, 2008.

Pal, Ashis B. "Implementation of a Geographic Information System (GIS) - The nuts and bolts." GIS Development. 2000.

http://www.gisdevelopment.net/technology/gis/techgi0012.htm (accessed December 9, 2009).

Presiado, Rhea, Deborah Elliot-Fisk, David Schirokauer, and David Press. Point Reys National Seashore/Golden Gate National Recreation Area Coastal Inventory Protocol. Thesis, Davis: University of California, 2005.

Ralston, Bruce. Developing GIS Solutions With MapObjects and Visual Basic. Albany, Tennesse: OnWord Press, 2002.

Schirokauer, David. Project Goals; email correspondence between David Schirokauer and Joseph Kinyon. Point Reyes Station, January 8, 2003.

Sharman, Lewis, et al. NPS Alaska Coastal Resources Inventory and Mapping Field Protocol - The Polygon Method - Developed at Glacier Bay National Park and Preserver 1997-1998. Field Protocol, Gustavus, AK: National Park Service, 2002.

Siedsma, Andrea. "Shipyards angry over delays in oil tanker orders." San Diego Business Journal (CBJ L.P.), March 1999.

Simonyi, Charles. "Hungarian Notation." msdn Visual Studio Developer Center. November 1999. http://msdn.microsoft.com/enus/library/aa260976\%28VS.60\%29.aspx (accessed December 9, 2009).

Simpson, Alan. Access VBA Programming for Dummies. Hoboken: Wiley Publishing Inc., 2004.

Singleton, Carol. San Francisco to receive oil spill response equipment grants. December 15, 2009. http://www.dfg.ca.gov/news/news09/2009121501.asp (accessed December 15, 2009).

Sullivan, Louis. Kindergarten Chats and Other Writings. New York: Dover, 1979.

Tecumseh Group. Tek-Tips Forums. http://www.tek-tips.com/ (accessed December 9, 2009).

Tomlinson, Roger. Thinking About GIS: Information System Planning for Managers. Redlands, CA: ESRI Press, 2003.

Townsend, R., and M. Glazer. Safe Passage: Preventing Oil Spills in our Marine Sanctuaries. Analysis, Washington, DC: Center for Marine Conservation, 1994.

Transportation Economics and Management Systems, Inc. Impact of High Oil Prices on Freight Transportation: Modal Shift Potential in Five Corridors -- Technical Report. Transportation Analysis, Washington, D.C.: Maritime Administration, U.S. Department of Transportation, 2008.

U.S. Department of Transportation Maritime Administration. Compilation of Maritime Laws: As ammended by the First Session of the 109th Congress. Washington, D.C., January 2008.

U.S. Energy Information Administration. "Short-Term Energy Outlook--December 2009; Full Report." EIA-Short-Term Energy Outlook. December 8, 2009. http://www.eia.doe.gov/emeu/steo/pub/contents.html (accessed December 9, 2009). 
U.S. Fish \& Wildlife Service. "National Wetlands Inventory; Download Seamless Wetlands Data by State." The U.S. Fish \& Wildlife Service . December 4, 2009. http://www.fws.gov/Wetlands/Data/DataDownloadState.html (accessed December 9, 2009).

United States Congress. "Title 33 -- NAVIGATION AND NAVIGABLE WATERS; CHAPTER 40 -- OIL POLLUTION." United Stated Code: Browse Titles Page. August 1990. http://frwebgate.access.gpo.gov/cgibin/usc.cgi?ACTION=BROWSE\&TITLE=33USCC40 (accessed December 9, 2009).

United States General Accounting Office. Maritime Industry: As U.S. Single-Hull Oil Vessels Are Eliminated, Few Double-Hull Vessels May Replace Them. Report to Congressional Requesters, Washington, D.C.: Government Printing Office, 2000. UtterAccess. UtterAccess Discussion Forums. http://www.utteraccess.com/forums/faq.php (accessed December 9, 2009). Welch, Craig. "Nation \& World | Oil tankers slow to shift to anti-spill double hulls." Seattle Times. May 5, 2003.

http://community.seattletimes.nwsource.com/archive/?date=20030505\&slug=tank er05m (accessed December 9, 2009).

Wiegers, Karl E. "Software Requirements Specification Template." Process Impact. 1999. http://www.processimpact.com/goodies.shtml (accessed December 2009, 2009). 


\section{Appendix}

There were several documents created or referenced as part of the creation of the database and interface. This section will provide the name of the document referenced above, a short summary of its content and the path to its location relative to the root directory. These documents are embedded within the structure of the database and are duplicated in the digital data set that accompanies this document. Essential to understanding the project and the references made in this document, the supporting documents total several hundreds of pages of content and are too large to reproduce as a paper record attached to the paper version of this document. These documents reflect a significant amount of work completed to facilitate the database and interface design.

General instructions to the location digital format appendix documents:

The root directory of the digital files accompanying this document contains two main directories and an html format text file of all the digital files contained within those directories.

The directory “Coastal_Biophysical_Inventory” contains the complete database (Microsoft Access 2003 format database and interface, file database, and spatial data), supporting documents, instructions for use, and the installation software that make up the project deliverables.

The directory "MIP_Presentation_Report” contains the electronic versions of this document, the PowerPoint file that accompanied defense of the project to the academic committee, and the directory "Appendices" that contain electronic versions of all the appendix items summarized and referenced on the following pages.

+[Root Directory] Joseph_Kinyon_Cohort4_UofR_MS_GIS_MIP_CBI_NPS_2010

[Directory] Coastal_Biophysical_Inventory

[Directory] MIP_Presentation_Report

Read_Me_Contents.html

Main directory diagram:

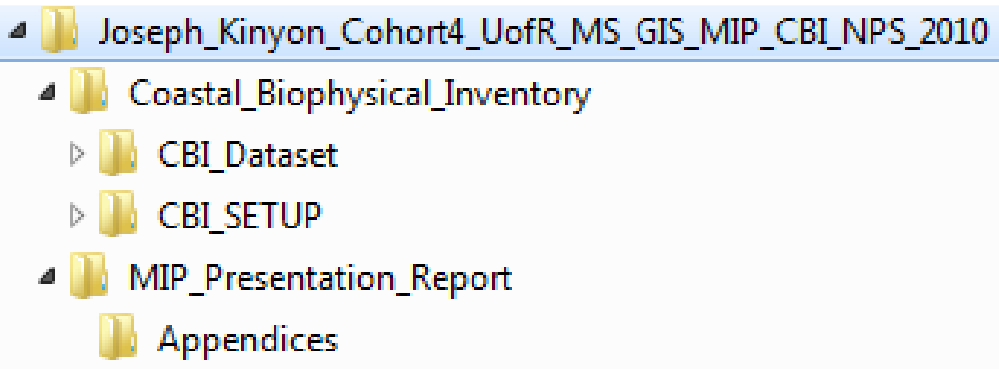




\begin{tabular}{|l|l|}
\hline Appendix A. & \\
\hline Document Name & Coastal Biophysical Inventory (CBI) and Mapping Protocol \\
\hline Author & Joseph Kinyon, Rani Gaddam \\
\hline Pages & 57 \\
\hline File Name & CBI_Protocol_FinalDraft_20100416.pdf \\
\hline Summary of Content & $\begin{array}{l}\text { Methodology for field data collection. Includes instructions for } \\
\text { characterization of physical features such as substrate, biological } \\
\text { observation methods, mapping of observed coastline, and data } \\
\text { management for observation records. }\end{array}$ \\
\hline
\end{tabular}

\begin{tabular}{|l|l|}
\hline Appendix B. & \\
\hline Document Name & $\begin{array}{l}\text { Coastal Biophysical Inventory 2.3 } \\
\text { Introduction to Data Entry } \\
\text { and Explanation of User Interface and Tools }\end{array}$ \\
\hline Author & Joseph Kinyon \\
\hline Pages & 87 \\
\hline File Name & CBI_Data_Entry_Guide_20100201.ppt \\
\hline Summary of Content & $\begin{array}{l}\text { Instructions for use of database interface for browsing and data } \\
\text { entry. }\end{array}$ \\
\hline
\end{tabular}

\begin{tabular}{|l|l|}
\hline Appendix C. & \\
\hline Document Name & $\begin{array}{l}\text { Coastal Biophysical Inventory Database Table } \\
\text { Relationship Diagram }\end{array}$ \\
\hline Author & Joseph Kinyon \\
\hline Pages & 1 (17 x 22 inches) \\
\hline File Name & Relationships_CBI_OBSERVATIONS_PCSLC_NPS.pdf \\
\hline Summary of Content & $\begin{array}{l}\text { Diagram illustrating the tables and relationship design } \\
\text { implemented in the database }\end{array}$ \\
\hline
\end{tabular}




\begin{tabular}{|l|l|}
\hline Appendix D. & \\
\hline Document Name & Coastal Biophysical Inventory Map Set \\
\hline Author & Joseph Kinyon \\
\hline Pages & 9 \\
\hline File Name & CBI_Maps.pdf \\
\hline Summary of Content & A set of maps illustrating summary analysis of collected data. \\
\hline
\end{tabular}

\begin{tabular}{|l|l|}
\hline Appendix E. & \\
\hline Document Name & $\begin{array}{l}\text { Exploratory analysis to examine robustness of CBI dataset: } \\
\text { Impacts of tide, survey team, and season }\end{array}$ \\
\hline Author & Ben Becker, Rani Gaddam, Joseph Kinyon \\
\hline Pages & 6 \\
\hline File Name & ExploratoryAnalysisToExamineRobustnessOfDataset_v15.pdf \\
\hline Summary of Content & $\begin{array}{l}\text { A statistical analysis of the observation data set based on } \\
\text { variables that could affect consistency of data. }\end{array}$ \\
\hline
\end{tabular}

\begin{tabular}{|l|l|}
\hline Appendix F. & \\
\hline Document Name & $\begin{array}{l}\text { Methods for processing Coastal Biophysical Inventory (CBI) } \\
\text { field coordinates into line features of the coast. }\end{array}$ \\
\hline Author & Joseph Kinyon \\
\hline Pages & 6 \\
\hline File Name & CBI_GPS_data_SegmentCreation.pdf \\
\hline Summary of Content & $\begin{array}{l}\text { Instructions to create CBI segment spatial features using GPS } \\
\text { coordinates and coastline line feature. This is workflow for } \\
\text { creating the base reference data for the GIS database. }\end{array}$ \\
\hline
\end{tabular}

\begin{tabular}{|l|l|}
\hline Appendix G. & \\
\hline Document Name & Coastal Biophysical Inventory Error Log \\
\hline Author & Rani Gaddam \\
\hline Pages & 4 \\
\hline File Name & error_log_121908.doc \\
\hline Summary of Content & $\begin{array}{l}\text { Communication between database user and database manager } \\
\text { about performance, bugs and feature requests. }\end{array}$ \\
\hline
\end{tabular}

